Semiclassical

Approach

to Relativistic Nuclear

Mean Field Theory

M. CENTELLES

Departament d'Estructura i

Constituents de la Matèria

Universitat de Barcelona

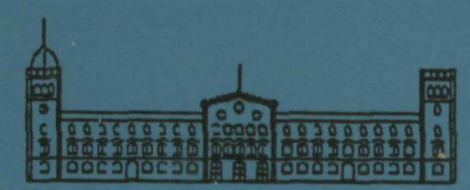




\section{Semiclassical Approach to Relativistic Nuclear Mean Field Theory}

Memòria presentada per

Mario Centelles Aixalà

per optar al grau de

Doctor en Ciències Físiques.

Barcelona, juliol 1992.

Departament d'Estructura i

Constituents de la Matèria.

Facultat de Física.

Universitat de Barcelona. 


\section{Semiclassical Approach to Relativistic Nuclear Mean Field Theory}

M. CENTELLES

University of Barcelona 
En memòria del meu pare.

Dedicat a la meva mare. 
I tu què et proposes esdevenir: déu, geni, sant, emperador?...

Pere Calders,

Gaeli i l'Home Déu.

En este caserío soñó sus primeras aventuras y rompió los primeros pantalones.

Pío Baroja,

Zalacaín el Aventurero.

We know a great many interesting details, learn new ones every week. But to construct a clear, easily comprehensible picture on which all physicists would agree-that is simply impossible.

ERWIN Schrödinger,

Scientific American 189 (3) (1953). 


\section{Contents}

Preface $\quad$ xi

Acknowledgements xiii

1 Introduction 1

2 Semiclassical Expansion in Relativistic Mean Field Theory 9

2.1 Expansion of the Propagator for Hamiltonians with Matrix Structure . 10

2.1.1 Statement of the problem . . . . . . . . . . . 10

2.1 .2 The method . . . . . . . . . . . . . . 11

2.2 Wigner Representation of the Propagator for a Scalar-Vector Dirac

Hamiltonian . . . . . . . . . . . . . . . . . 14

2.2.1 Wigner transform of the Dirac hamiltonian . . . . . . . . 14

2.2.2 Wigner transform of the relativistic propagator $\ldots \ldots \ldots 16$

2.2.3 Connection with other approaches . . . . . . . . 18

2.3 The Relativistic Wigner-Kirkwood Density Matrix . . . . . . . . 20

2.4 Semiclassical Relativistic Particle and Energy Densities . . . . . . . 22

2.4.1 Wigner-Kirkwood expressions . . . . . . . . . 23

2.4 .2 Density functionals $\ldots \ldots \ldots \ldots \ldots \ldots$ 
3 Test Calculations with a Relativistic Harmonic Oscillator 30

3.1 Solution of the Dirac Equation . . . . . . . . . . . . . . 31

3.2 Wigner-Kirkwood Expansion of the Particle Number and the Energy . 33

3.3 Discussion of Results . . . . . . . . . . . . . . . . 35

4 Semiclassical Description of Nuclear Systems in Relativistic Mean Field Models $\quad 42$

4.1 Overview . . . . . . . . . . . . . . . . . . 43

4.2 Calculations of Finite Nuclei . . . . . . . . . . . . . . 45

4.2 .1 Lagrangians and energy densities . . . . . . . . . . 45

4.2 .2 Variational equations . . . . . . . . . . . . . 49

4.2 .3 Numerical solution . . . . . . . . . . . . . . . . . 51

4.2 .4 Results and discussion $\ldots \ldots \ldots \ldots \ldots \ldots$

4.3 Liquid Drop Model Coefficients . . . . . . . . . . . . . 71

5 Relativistic Extended Thomas-Fermi Calculations of Finite Nuclei with Realistic Nucleon-Nucleon Interactions $\quad 76$

5.1 The Energy Density Functional . . . . . . . . . . . . . 78

5.1.1 The Dirac-Brueckner-Hartree-Fock potential energy density . . 78

5.1.2 The complete energy density functional and parameter fit . . . 80

5.2 Fission Barriers . . . . . . . . . . . . . . . . . . 85

5.3 Heavy Ion Optical Potentials at Intermediate Energies . . . . . . . 92

$\begin{array}{ll}\text { Summary and Concluding Remarks } & 101\end{array}$

$\begin{array}{ll}\text { Appendix A } & 107\end{array}$

$\begin{array}{ll}\text { Appendix B } & 110\end{array}$

$\begin{array}{ll}\text { Appendix C } & 113\end{array}$

$\begin{array}{ll}\text { Bibliography } & 115\end{array}$ 


\section{Preface}

INITIATING in the second half of 1989 , this thesis is the outcome of the main part of the research work carried out by the author within the nuclear physics group of the Departament d'Estructura i Constituents de la Matèria of the University of Barcelona. On the basis of Wigner transform techniques, we set up the semiclassical expansion to order $\hbar^{2}$ in relativistic nuclear mean field theory. Our study represents an extension of the well-known non-relativistic semiclassical theory to relativistic nuclear physics, where the Dirac structure poses several complications which make the formalism and numerical treatment substantially more difficult.

To some extent, the contents and layout of this thesis lean on material submitted for publication or already published in specialized journals, although we here touch and discuss several matters and results not reported in those contributions. Following an introduction in Chapter 1, Chapter 2 develops the subject matter of the thesis. In this chapter we formulate the theoretical formalism and concentrate on the derivation of the relativistic Wigner-Kirkwood expansions and density functional expressions. Chapter 3 is devoted to analyze the semiclassical approach in the model problem of a relativistic harmonic oscillator. In Chapters 4 and 5 we shall use the derived density functionals to study realistic situations within relativistic nuclear physics, namely finite nuclei and semi-infinite nuclear matter in the context of the non-linear $\sigma-\omega$ model, and the description of finite nuclei, fission barriers and heavy ion scattering based on a local density approximation to microscopic Dirac-Brueckner self-energies obtained in nuclear matter. These applications will serve to emphasize the physical picture behind the theoretical exposition and the reliability of the semiclassical scheme. A summary of 
of the main conclusions and perspectives is presented in the last chapter. Finally, some special topics and derivations are treated in the appendices.

Throughout this work, bold-faced symbols indicate vectors in a three-dimensional space. Those quantities which are operators are denoted by a circumflex, e.g. $\hat{H}$, while the corresponding classical quantity appears without special designation, e.g. $H$. We set $\hbar=c=1$ everywhere unless $\hbar$ is written explicitly for the sake of clarity in the semiclassical expansions.

I have opted for writing up this thesis in a language other than my mother tongue with the will that it may have a chance to reach a wider audience. I hope that the reader does not suffer too much from my insufficient proficiency and fluency in the English usage. 


\section{Acknowledgements}

THE development of this project has left me increasingly indebted to many. I owe major debt to Xavier Viñas under whose direction this thesis has grown, he introduced me into the subject and his continuous collaboration in the investigations has made them possible. His dedication and support-and indefatigable forbearance-deserve my sincere gratitude. I extend deep recognition to Manuel Barranco, who has also contributed to this project and has substantially influenced the progress of my work with constant and invaluable interest in it.

I take pleasure in expressing great appreciation to Prof. Peter Schuck, with whom I was privileged to collaborate at several phases of this work. I am deeply beholden to Martí $\mathrm{Pi}$ whose expert counselling and assistance have played a key role in developing the computer codes I employed. I benefited greatly during this time from stimulating collaboration with Francesca Garcias, Saturnino Marcos, Roland J. Lombard, Dao T. Khoa and Prof. Herbert Müther. I wish to record my thanks to Joan Martorell, Michel Farine, Detlef Von-Eiff and Prof. Manfred K. Weigel for valuable discussions.

I gratefully acknowledge the Departament d'Estructura i Constituents de la Matèria of the University of Barcelona for receiving me and for providing facilities to carry out my labour, and the Departament d'Ensenyament de la Generalitat de Catalunya for a grant during the first stages of this work.

The final tribute goes to my colleagues, the elder and the younger, from the atomic and nuclear physics group. They have helped so much to make this task a pleasant one daily, that it is difficult to adequately express the authentic feeling of esteem to them. $A$ tots vosaltres qui $m$ 'heu dispensat la vostra amistat, gràcies. 


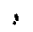




\section{Chapter 1}

\section{Introduction}

IN THE year 1953, an article entitled "What Holds the Nucleus Together?" by Hans A. Bethe was published in Scientific American [Be53]. Since the discovery of the atomic nucleus and its constituents in the first third of this century, the question formulated by Bethe has been a primary problem in physics and remains only partially answered at present. Nevertheless, the challenge for the nuclear physicist does not end there. Once a model for the interaction between the nucleons has been assumed, the difficult programme of investigating the nuclear properties in terms of the underlying interaction arises.

Finite nuclei are complex many-body systems. Any attempt to describe these objects within a single and fundamental theory becomes, from the outset, too ambitious an undertaking. To overcome the many obstacles that are to be faced, approximation schemes that make the problem more tractable have to be adopted. The conventional approach is based on a non-relativistic formulation. Relativistic effects were considered as being negligible, although it always was clear that nucleons are in principle Dirac particles: the mean value of the nucleon velocity in the nuclear medium can be as large as forty per cent of the velocity of light. Traditionally, the description of nuclear structure has been approached by solving a many-body Schrödinger equation which involves nucleons interacting through static nucleon-nucleon (NN) potentials. This has been done within the Hartree-Fock (HF) approximation, where the many-body wave 
function of the system is replaced by a Slater determinant of single-particle wave functions obtained in a self-consistent way from the mean field produced by the nucleons themselves.

In many non-relativistic models, the free two-nucleon interaction is determined from NN scattering data together with the properties of the deuteron. After Yukawa's original theory, it is generally recognized that the NN interaction is mediated by the exchange of mesons [Ma89] (even though, nowadays, meson theory is already understood as an effective description of the underlying quark-gluon dynamics in the low-energy regime). To test the various approximations and assumptions of the theory, and to investigate the nature of the nuclear force, the mechanism of saturation, or the role of many-body effects, nuclear physicists chose to study infinite nuclear matter. Major progress has been made in the microscopic approach of this system, mainly along the lines of the Brueckner-Bethe-Goldstone many-body theory [Sp72, Ma89]. The problem of saturation in finite nuclei in non-relativistic Brueckner-Hartree-Fock (BHF) calculations has been revisited recently [Sc91].

A quantitative microscopic description of real nuclei, however, has so far only been possible on a phenomenological level. Effective interactions depending on the density have to be constructed in order to account for ground-state properties. In some studies these effective forces are determined from a local density approximation (LDA) to $\mathrm{BHF}$ calculations, with additional dependence on the density to simulate the effect of the omitted higher-order corrections [Ne70, Fr75]. Quite successful approximations are purely phenomenological density-dependent interactions, like the (zero range) Skyrme force [Sk56, Va72] or the (finite range) Gogny force [Go75]. These forces may be understood as effective parametrizations of the $G$-matrix. With only a few free adjustable parameters to reproduce densities and binding energies for nuclear matter and for spherical closed shell nuclei, these forces have shown to be able not only to yield excellent nuclear ground-state properties of spherical as well as of deformed nuclei, but also to describe on a quantitative level nuclear dynamical phenomena, like fission, heavy ion collisions at low and intermediate energies or nuclear excitation spectra, using methods like time-dependent $\mathrm{HF}$ or the random phase approximation. See e.g. [F175] and the review articles [Qu78] and [Ne82] for an account of this density-dependent Hartree-Fock (DDHF) scheme.

Despite the remarkable success achieved by these sophisticated non-relativistic calculations, discrepancies still remain with experimental data indicating that such a 
traditional treatment is reaching its limits. Open problems are the understanding of the large single-particle spin-orbit interaction in nuclei, the fact that DDHF calculations yield too strong oscillations in the interior of the charge density distributions, the correct prediction of spin-dependent effects in scattering experiments, or the quantitative explanation of nuclear saturation (the density at saturation is always too high for reasonable binding energies).

All of these shortcomings stimulated the investigation of alternative approaches during the last years. We reproduce in Table 1.1 a table from [Ma89] that illustrates some possible extensions which go beyond the conventional assumptions concerning some quantities relevant to the nuclear many-body problem. In analogy to an idea which was born already a long time ago [Jo55, Du56], Walecka and coworkers developed quantum hadrodynamics (QHD), a relativistic quantum field theory for the nuclear many-body problem [Wa74, Ch74, Se86]. It was originally conceived to describe the behaviour of the equation of state for dense nuclear matter, a subject of great interest in the fields of heavy ion physics at high energies and of astrophysics. In the formalism of QHD, baryonic and mesonic degrees of freedom are treated on an equal footing. Nucleons interact through the exchange of virtual mesons, so that static potentials need not to be introduced and the effects of relativistic propagation are naturally incorporated. In the simplest version, only a vector field accounting for the short range repulsion and a scalar field responsible for attraction are needed to describe saturation in nuclear matter. The scalar and vector fields may be associated with the $\sigma$ and $\omega$ mesons, respectively.

Of course, the full theory of QHD is highly complex and suitable approximations are needed. In practice, one also deals with a phenomenological approach. The coupling constants and some meson masses of the effective meson-nucleon Lagrangian are taken as free parameters, which are adjusted to fit the properties of nuclear matter and finite nuclei. Most calculations have been done in mean field Hartree approximation, neglecting exchange terms and without including any contribution from antiparticles [Ho81b, Re86, Ga90a]. For a review of relativistic nuclear mean field theory, we refer the reader to [Se86] and [Re89]. These so-called $\sigma-\omega$ models constitute, in a sense, the relativistic analog of the non-relativistic DDHF method. It may be stated [Re86, Ga90a] that relativistic mean field models of reasonable sophistication achieve about the same agreement with experiment compared with the more elaborated densitydependent interactions in the non-relativistic HF approximation, with the conceptual 
Table 1.1. Basic assumptions underlying the nuclear many-body problem and possible extensions.

\begin{tabular}{|c|c|c|}
\hline Item & Simplest assumption & Possible extensions \\
\hline \multirow[t]{3}{*}{ Degrees of freedom } & Nucleons only & Mesons \\
\hline & & Isobars \\
\hline & & Quarks and gluons \\
\hline Hadron structure & Point structure & (Quark) substructure \\
\hline \multirow[t]{2}{*}{ Interaction } & (Static, instantaneous) & Non-static interactions \\
\hline & two-body potential & Many-body forces \\
\hline \multirow[t]{2}{*}{ Dynamical equation } & Non-relativistic & Relativistic \\
\hline & Schrödinger equation & Dirac equation \\
\hline
\end{tabular}

advantage of being fully relativistic and thus automatically incorporating the spin-orbit force, which is of fundamental importance in nuclear structure physics.

More sophisticated approaches have also been investigated in the relativistic framework. Dirac-Hartree-Fock calculations of nuclear matter and finite nuclei are available [Bo87, Bl87, Lo88]. Dirac-Hartree-Fock-Bogoliuvob equations to treat pairing correlations have been derived [Ku91a]. The microscopic Dirac-Brueckner-HartreeFock theory [An83, Ce86, Ha87, Br90, Mü90] has shown that the old puzzle of the non-relativistic Coester band can be eschewed, and that the empirical saturation and incompressibility of nuclear matter can be reproduced if the theory incorporates the effects of the density-dependent Dirac spinor for the nucleons inside the medium.

However, most of these applications are, as their non-relativistic counterpart, quite involved on a purely quantum mechanical level. In non-relativistic nuclear physics, semiclassical models have become very popular [Ha84, Dr85, Ar87] due to their reliability and feasibility, as they avoid the task of solving the quantal mean field HF equations. Most commonly, these methods are based on the Wigner-Kirkwood (WK) $\hbar$ expansion [Wi32, Ki33, Uh36] of the density matrix albeit alternative formulations, somehow related, are also available in the literature. Among others we cite, for instance, partial resummation and saddle-point techniques, see [Ri80] for a brief account of these methods, the gradient expansion of Kirzhnits [Ki67], the Green's function approach of Balian and Bloch [Ba71], or expansions based on the standard WKB method 
[Kr90]. Indeed, the semiclassical approach, together with density functional theory, has become a powerful tool for treating inhomogeneous Fermi systems and it is widely used in a number of fields in physics and chemistry [Lu83, Dr85, Jo89, Dr90, Pe91] (recent thriving applications are, e.g., metallic clusters and quantum liquids).

In the semiclassical extended Thomas-Fermi (ETF) method [Bo76a, Br85] (and references quoted therein, see also [Tr86]), the total energy of a nucleus is expressed as a functional of the local particle density and its gradients. The ground-state density distribution $\rho$ is found from the energy density functional $\mathcal{E}[\rho]$ by a variational approach:

$$
\frac{\delta}{\delta \rho} \int d \boldsymbol{r}\{\mathcal{E}[\rho(\boldsymbol{r})]-\lambda \rho(\boldsymbol{r})\}=0,
$$

a technique that was early used in nuclear physics [Be56].

The theoretical justification of this so-called energy density formalism [Br68a, Lo73] is given by the Hohenberg-Kohn theorem [Ho64] (generalized to finite temperature by Mermin [Me65]). For nuclear physics, the theorem states that the exact ground-state energy $E$ of a nucleus can be expressed as a unique functional of the local ground-state density $\rho$ alone,

$$
E=\int d \boldsymbol{r} \mathcal{E}[\rho(\boldsymbol{r})]
$$

This furthermore implies that the variational principle (1.1) leads to the exact solutions $E$ and $\rho$, if the exact functional $\mathcal{E}[\rho]$ is used. Unfortunately, the exact functional is not known and one works with approximate asymptotic expansions in terms of gradients of the density as in the ETF method.

To cure the deficiencies of the lowest-order Thomas-Fermi approximation, providing a more accurate description of the nuclear surface, at least corrections of order $\hbar^{2}$ have to be considered. In the most elaborated versions, the non-relativistic ETF density functionals include gradient corrections coming from inhomogeneity and non-local effects (e.g., spin-orbit and effective mass terms) up to order $\hbar^{4}$ [Br76, Gr79, Gr80]. The corresponding theory at finite temperature has also been derived [ $\mathrm{Br} 85, \mathrm{Ba} 85]$. A priori, the continuation to include $\hbar^{6}$-order terms should be straightforward. But semiclassical expansions are asymptotical and the $\hbar^{6}$-order functional [Mu81] leads to a term which for large distances goes like $\rho^{-1 / 3}$, and thus diverges when the density $\rho$ falls to zero.

The Thomas-Fermi model and its extensions represent the semiclassical treatment of fermions in a nucleus in the independent particle or HF approximation. The ETF 
method smoothes out quantal effects and one is left with the average part of the HF energy, which is similar in spirit to the droplet model [My69, My74]. The remaining part of the energy, much smaller, is of pure quantal origin and can be added perturbatively to the semiclassical smooth quantity, as indicated by the Strutinsky energy theorem [St67, St68, Br81] or by the so-called expectation value method [Bo76a, Br85].

In non-relativistic nuclear physics, the ETF method has been mainly used together with Skyrme or Gogny forces. Detailed ETF calculations with Skyrme forces in comparison with the corresponding $\mathrm{HF}$ results can be found in [Br85] and [Ce90a]. Many studies confirm that semiclassical Thomas-Fermi and ETF models, at zero or at finite temperature, can be very useful in different areas of nuclear physics. For example, they have been used to deal with the description of excited hot nuclei [Ba81a, Ba81b, $\mathrm{Ba} 85, \mathrm{Br} 85$, Su87], of supernova matter [Ma82, Pi86], of the interaction potential in heavy ion scattering [Br68b, $\mathrm{Ng} 75, \mathrm{Gu} 92]$, or in static constrained calculations of giant resonances [Su88, Li89, Ce90a, Gl90], deformation energies and fission barriers [Br85, Da85a, Ga89a, Ga91]. It is worth mentioning that the domain of application of semiclassical methods is not restricted to static properties of the nucleus only. The semiclassical approach has also been generalized to the description of nuclear dynamics, usually on the basis of the Landau-Vlasov equation, giving rise to a field which is at present still the subject of intensive studies [Ri80, $\operatorname{Pr} 83, \mathrm{Gr} 87, \mathrm{Be} 88, \mathrm{Sc89}$, Su89].

The success of semiclassical models in non-relativistic physics provides a very strong motivation for investigating similar methods in the relativistic context. The relativistic Thomas-Fermi model was formulated by Vallarta and Rosen in the early thirties [Va32]. The basic theorem of Hohenberg and Kohn was extended to the relativistic domain (see e.g. [Ra73, Do79, Ra83]), thus setting the foundations of relativistic density functional theory. Relativistic $\hbar$ corrections to the Thomas-Fermi approximation have been introduced in atomic physics on the basis of a field theoretical formulation by Dreizler and collaborators [Dr90]. In the first instance, these authors used the gradient expansion method of Kirzhnits [Ki67] for the derivation of such corrections (Gross and Dreizler in [Dr85], p. 81, and [Ma86, En87]). Later, they introduced an alternative approach based on Green's function techniques [En89, Mü89], which has allowed them to calculate the fourth-order gradient corrections to the non-interacting kinetic energy density of a relativistic system of electrons and positrons [Po91].

Until very recently, semiclassical methods applied to relativistic nuclear physics were at a less developed stage. For instance, the $\sigma-\omega$ model had only been solved in 
the simplest Thomas-Fermi approximation for finite nuclei [Bo77a, Se78, Se79, Se86] and for semi-infinite nuclear matter [Bo77b, St91, Sh91a, Sh91b]. With respect to the case of atomic physics, the derivation of $\hbar$ corrections in the nuclear problem has the additional difficulty of the existence of a position-dependent effective mass, originating from the scalar potential, which renders the semiclassical treatment and expressions much more involved.

In [Ce90b] we have given for the first time the semiclassical relativistic expressions of the particle and energy densities, including $\hbar^{2}$-order gradient corrections, for a set of fermions moving in the mean field arising from a scalar potential and from the time-like component of a four-vector potential. In [Ce92c] we have presented a comprehensive description of the formalism of this semiclassical expansion. The method is based on the Wigner transform of operators and affords a transparent and straightforward $\hbar$ expansion of the propagator, valid for time-independent single-particle hamiltonians with a matrix structure. The semiclassical picture would be somehow incomplete if the study were only confined to formal aspects of the theory, without exploring its versatility in actual applications. To this end, we have investigated the solution of the relativisitc variational equations corresponding to the $\hbar^{2}$ expansion. In [Ce92a, Ce92c] we have performed calculations of finite nuclei and semi-infinite nuclear matter within non-linear $\sigma-\omega$ models, in mean field Hartree approximation, and have compared the semiclassical results with the ones obtained from the corresponding quantal calculation. In [Ce91, Ce92b] we have studied the structure of finite nuclei, fission barriers and the optical potential for heavy ion scattering, using the relativistic kinetic energy density of order $\hbar^{2}$, together with a potential energy density obtained from a local density approximation to Dirac-Brueckner calculations.

More effort has been devoted to the development of semiclassical expansions in the relativistic nuclear framework. Weigel, Haddad and Weber [We91] have used the Green's function scheme and the Wigner representation to obtain in the HF approximation $\hbar^{2}$-order WK expressions for a Lagrangian of one-boson-exchange potential structure. A derivation of the semiclassical approximation to the relativistic finite temperature HF method has been given by Von-Eiff and Weigel [Ei91a]. A recent and extensive investigation on the density functional approach to QHD has been carried out by Speicher, Dreizler and Engel [Sp92]. These authors have shown the validity of the Hohenberg-Kohn theorem in QHD. On the basis of the expansion techniques of [En89] for the Green's function, the semiclassical non-interacting kinetic energy density 
has been derived to order $\hbar^{2}$, taking into account vacuum contributions and the four components of the vector potential. The specific case of the $\sigma-\omega$ model in Hartree approximation is also addressed by these authors. The extension of this density functional description of QHD to finite temperature has been formulated by Müller and Dreizler very recently [Mü92]. However, in all of these studies no numerical applications have been made.

We finally remark that we have in no sense tried to be exhaustive and complete in the short review made in this introduction; this would be out of the scope of this work. It was intended only to bring out the essence of the topics we shall be dealing with later, to give a brief overview of the past and present work in the field, as well as to set the stage for the presentation of our original formalism and its applications, which constitutes the true objective of this thesis. There is an unavoidable element of arbitrariness in the choice of what to include in the text and how to structure it. In what follows, our aim has been to provide clear and balanced coverage of the theory and the applications, with as much interest and as little pain for the reader as possible. 


\section{Chapter 2}

\section{Semiclassical Expansion in Relativistic Mean Field Theory}

IN THIS chapter we shall introduce and develop the theoretical formalism on which the subsequent chapters will rest. We shall present in mean field approximation the formal structure of the relativistic semiclassical expansion, that will be derived from the propagator in the Wigner representation. We begin by introducing a method based on the Wigner transform of operators to perform the $\hbar$ expansion of the propagator for hamiltonians which have a matrix structure. We then turn our attention to the relativistic nuclear problem, and apply the method to a Dirac hamiltonian which contains a scalar field and the time-like component of a Lorentz-vector field. The propagator is related to the density matrix and its connection with ground-state averages of physical quantities, viz. particle and energy densities, is shown. In the next step, the WignerKirkwood expansion of these quantities is obtained to order $\hbar^{2}$. Finally, we substitute in favour of the particle density in the Wigner-Kirkwood expressions to obtain the corresponding $\hbar^{2}$-order density functionals, which we shall refer to as constituting the relativistic extended Thomas-Fermi (RETF) method. 


\subsection{Expansion of the Propagator for Hamiltonians with Matrix Structure}

\subsubsection{Statement of the problem}

In Quantum Mechanics the problem described by a time-independent single-particle hamiltonian $\hat{H}$ has an associated propagator defined by

$$
\hat{G}(\eta)=\exp (-\eta \hat{H})
$$

The parameter $\eta$ is proportional to time, although it also can be interpreted as the inverse temperature in statistical physics.

For hamiltonians whose classical counterpart is a scalar in phase space, the $\hbar$ expansion of the propagator (2.1) is well-known [Ri80]. In fact, for this case the final objective being the $\hbar$ expansion of the density matrix, there exists a variety of procedures to arrive at the same result. We mention, for instance, the partition function approach of Bhaduri and collaborators [Bh71, Je75a, Je75b], the Kirzhnits expansion [Ki67, Ho73], the algebraic method of Grammaticos and Voros based on the Wigner transform of operators [Gr79, Gr80], or the direct $\hbar$ expansion of the density matrix $\hat{\rho}=\Theta(\lambda-\hat{H})[$ Ri80]. With hamiltonians of scalar nature, the choice of one of these apparently different derivations is a matter of taste and computational ease, for it can be argued that they are equivalent [Ba84].

However, in problems where the hamiltonian has a matrix structure, like the relativistic one, the task is much more involved due to the additional difficulty of the non-commutativity of the matrices entering $\hat{H}$. In this case, the above-mentioned methods are not suitable enough because they yield an infinite series in powers of $\eta$ separately for each order of the $\hbar$ expansion of $\hat{G}$. Moreover, they mix positive and negative energy states, producing strong cancellations in the series. Therefore, to get the sought-after $\hbar$ expansion one has to be capable of resumming the power series of $\eta$ for the positive and the negative energy solutions independently. Hence the interest to look for an alternative and more direct way to perform the semiclassical $\hbar$ expansion of the propagator when the system is described by a hamiltonian with matrix components. 


\subsubsection{The method}

Consider a time-independent single-particle hamiltonian $\hat{H}$ which has a matrix structure. The Bloch equation for the propagator is obtained from Eq. (2.1) by differentiation with respect to $\eta$ :

$$
\frac{\partial \hat{G}}{\partial \eta}=-\hat{H} \hat{G}=-\hat{G} \hat{H}
$$

To perform the semiclassical approximation of the Bloch equation ${ }^{\dagger}$ it is convenient to write the r.h.s. of $(2.2)$ in a symmetrized way:

$$
\frac{\partial \hat{G}}{\partial \eta}+\frac{1}{2}\{\hat{H}, \hat{G}\}=0
$$

where $\{\hat{H}, \hat{G}\}$ denotes the anticommutator of $\hat{H}$ and $\hat{G}$. The differential equation (2.3) has to be solved preserving the boundary condition

$$
\hat{G}(\eta=0)=I
$$

$I$ being the unit matrix. The Wigner transform [Wi32, Ri80] of Eq. (2.3) results in

$$
\frac{\partial G_{\mathrm{w}}}{\partial \eta}+\frac{1}{2}\left\{H_{\mathrm{w}}, \exp \left(\frac{i \hbar}{2} \vec{\Lambda}\right), G_{\mathrm{w}}\right\}=0
$$

where for $A_{\text {w }}$ we mean the Wigner transform of the operator $\hat{A}$, and

$$
\left\{A_{\mathrm{w}}, \exp \left(\frac{i \hbar}{2} \overleftrightarrow{\Lambda}\right), B_{\mathrm{w}}\right\} \equiv A_{\mathrm{w}} \exp \left(\frac{i \hbar}{2} \vec{\Lambda}\right) B_{\mathrm{w}}+B_{\mathrm{w}} \exp \left(\frac{i \hbar}{2} \overleftrightarrow{\Lambda}\right) A_{\mathrm{w}}
$$

for any two operators $\hat{A}$ and $\hat{B}$. In writing (2.5) we have made use of the following property of the Wigner transform of a product of operators [Ri80]:

$$
(\hat{A} \hat{B})_{\mathrm{w}}=A_{\mathrm{w}} \exp \left(\frac{i \hbar}{2} \overleftrightarrow{\Lambda}\right) B_{\mathrm{w}}
$$

where we have introduced the differential operator $\overleftrightarrow{\Lambda} \equiv \overleftarrow{\nabla}_{r} \cdot \vec{\nabla}_{p}-\overleftarrow{\nabla}_{p} \cdot \vec{\nabla}_{r}$ (the arrows indicate in which direction the gradients act).

'In this subsection we will follow a procedure which is similar to the one Taruishi and Schuck have employed to obtain a semiclassical BCS theory [Ta92]. 
If we write the Wigner transform of the Bloch propagator explicitly as a power series of $\hbar$,

$$
G_{\mathrm{w}}=\sum_{n=0} \hbar^{n} G_{n},
$$

expand $\exp (i \hbar \overleftrightarrow{\Lambda} / 2)$ and equate the coefficients of the same powers of $\hbar$, we obtain from Eq. (2.5) a set of coupled differential equations for the $G_{n}$ in the $\hbar$ expansion of -the propagator:

$$
\frac{\partial G_{n}}{\partial \eta}+\frac{1}{2} \sum_{m=0}^{n} \frac{1}{m !}\left(\frac{i}{2}\right)^{m}\left\{H_{\mathrm{w}},(\stackrel{\leftrightarrow}{\Lambda})^{m}, G_{n-m}\right\}=0, \quad n=0,1,2, \ldots
$$

At zero order:

$$
\frac{\partial G_{0}}{\partial \eta}+\frac{1}{2}\left\{H_{\mathrm{w}}, G_{0}\right\}=0
$$

which has the solution

$$
G_{0}=\exp \left(-\eta H_{\mathrm{w}}\right) .
$$

We will now show that from the knowledge of $G_{0}$ a recursive scheme can be worked out to obtain successively the higher-order corrections to the $\hbar$ expansion of the propagator. For the first order $G_{1}$, we have from (2.9)

$$
\frac{\partial G_{1}}{\partial \eta}+\frac{1}{2}\left\{H_{\mathrm{w}}, G_{1}\right\}+\frac{i}{4}\left\{H_{\mathrm{w}}, \stackrel{\leftrightarrow}{\Lambda}, G_{0}\right\}=0
$$

To solve this equation we proceed as follows. Since $G_{0}$ is an exponential, Eq. (2.11), any power of it is well defined:

$$
\left(G_{0}\right)^{s}=G_{0}(\eta \longrightarrow s \eta)=\exp \left(-s \eta H_{\mathrm{w}}\right) .
$$

Let us introduce an auxiliary matrix $\bar{G}_{1}$ defined through

$$
G_{1}=G_{0}^{1 / 2} \bar{G}_{1} G_{0}^{1 / 2} .
$$

After differentiation of this equation with respect to $\eta$

$$
\frac{\partial G_{1}}{\partial \eta}=-\frac{1}{2}\left\{H_{\mathrm{w}}, G_{1}\right\}+G_{0}^{1 / 2} \frac{\partial \bar{G}_{1}}{\partial \eta} G_{0}^{1 / 2}
$$

and combining Eq. (2.15) with Eq. (2.12) we get

$$
\frac{\partial \bar{G}_{1}}{\partial \eta}=-\frac{i}{4} G_{0}^{-1 / 2}\left\{H_{\mathrm{w}}, \stackrel{\leftrightarrow}{\Lambda}, G_{0}\right\} G_{0}^{-1 / 2} .
$$


By integrating this equation and using (2.14), one readily obtains

$$
G_{1}=-\frac{i}{4} G_{0}^{1 / 2}\left[\int_{0}^{\eta} d \eta^{\prime} G_{0}^{-1 / 2}\left\{H_{\mathrm{w}}, \overleftrightarrow{\Lambda}, G_{0}\right\} G_{0}^{-1 / 2}\right] G_{0}^{1 / 2}
$$

where the lower limit in the integral ensures that $G_{1}(\eta=0)=0$. Note that the boundary condition Eq. (2.4) implies $G_{\mathrm{w}}(\eta=0)=I$, and as $G_{0}(\eta=0)=I$ from (2.11), it must be

$$
G_{n}(\eta=0)=0 \quad \forall n>0
$$

The procedure we have presented for $G_{1}$ can be easily generalized to get any order $G_{n}$ when the $G_{m}$ with $m<n$ are known. In analogy to (2.14) we define

$$
G_{n}=G_{0}^{1 / 2} \bar{G}_{n} G_{0}^{1 / 2}
$$

which differentiated with respect to $\eta$ and combined with Eq. (2.9) leads to

$$
\frac{\partial \bar{G}_{n}}{\partial \eta}=-\frac{1}{2} G_{0}^{-1 / 2}\left[\sum_{m=1}^{n} \frac{1}{m !}\left(\frac{i}{2}\right)^{m}\left\{H_{\mathrm{w}},(\overleftrightarrow{\Lambda})^{m}, G_{n-m}\right\}\right] G_{0}^{-1 / 2}
$$

and finally:

$$
G_{n}=-\frac{1}{2} G_{0}^{1 / 2}\left[\sum_{m=1}^{n} \frac{1}{m !}\left(\frac{i}{2}\right)^{m} \int_{0}^{\eta} d \eta^{\prime} G_{0}^{-1 / 2}\left\{H_{\mathrm{w}},(\stackrel{\leftrightarrow}{\Lambda})^{m}, G_{n-m}\right\} G_{0}^{-1 / 2}\right] G_{0}^{1 / 2}
$$

One therefore ends up with a recursion relation which allows to obtain any desired $G_{n}$ in the $\hbar$ expansion of the propagator from the lowest order $G_{0}$. The problem has been reduced to an algebraic question whose main complexity will be the calculation of the symmetric action of the powers of $\overleftrightarrow{\Lambda}$ between $H_{\mathrm{w}}$ and the lower $G_{n}$.

The use of the propagator has the advantage that it not only yields the density matrix as we will show subsequently but, if $\eta$ is interpreted as an inverse temperature, it also yields the partition function of statistical mechanics, which is represented by $\hat{G}$ up to a norm. However, we have to stress the point that here we always deal with a system at zero temperature. 


\subsection{Wigner Representation of the Propagator for a Scalar-Vector Dirac Hamiltonian}

Our aim is to apply the method outlined above to obtain the semiclassical expansion up to order $\hbar^{2}$ of the propagator associated with a Dirac hamiltonian which describes the single-particle motion of a set of non-interacting fermions submitted to a scalar field $S(\boldsymbol{r})$ and to the time-like component $V(\boldsymbol{r})$ of a four-vector field, as in the case of relativistic mean field $\sigma-\omega$ models. Defining an effective mass

$$
m^{*}(\boldsymbol{r})=m+S(\boldsymbol{r})
$$

such a hamiltonian reads

$$
\hat{H}=\boldsymbol{\alpha} \cdot \hat{\boldsymbol{p}}+\beta m^{*}(\boldsymbol{r})+I V(\boldsymbol{r})
$$

where $\alpha$ and $\beta$ are the standard $4 \times 4$ Dirac matrices [Bj64]. According to the plan drawn in the preceding section, we must start by calculating the Wigner transform of Eq. (2.23).

\subsubsection{Wigner transform of the Dirac hamiltonian}

We proceed to show that in the Wigner representation the Dirac hamiltonian (2.23) is equal to its classical counterpart

$$
H=\boldsymbol{\alpha} \cdot \boldsymbol{p}+\beta m^{*}+I V
$$

The Wigner transform of a single-particle operator $\hat{A}\left(x, x^{\prime}\right)$ in Minkowski space is defined by

$$
A_{\mathrm{w}}=A_{\mathrm{w}}(q, p)=\int d s e^{i p \cdot s}\langle q+s / 2|\hat{A}| q-s / 2\rangle
$$

where $x$ and $x^{\prime}$ are four-vectors, $p$ is the four-momentum, $q=\left(x+x^{\prime}\right) / 2$ and $s=x-x^{\prime}$. If $\hat{A}$ is time-independent (2.25) becomes

$$
A_{\mathrm{w}}=\int d s e^{-i p \cdot s}\langle q+s / 2|\hat{A}| q-s / 2\rangle
$$

We now consider the case where $\hat{A}$ is the Dirac hamiltonian:

$$
\hat{H}=\hat{t}+\beta S+I V
$$


with

$$
\hat{t}=\alpha \cdot \hat{p}+\beta m .
$$

It is straightforward to realize that $V_{\mathrm{w}}=V(\boldsymbol{q})$ and $S_{\mathrm{w}}=S(\boldsymbol{q})$, so that we only need to calculate $t_{\mathrm{w}}$.

The eigenstates of $\hat{t}$ are the plane waves solution of the free Dirac equation:

$$
\hat{t}|\boldsymbol{p} \sigma e\rangle=e \tilde{\varepsilon}|\boldsymbol{p} \sigma e\rangle,
$$

where $\tilde{\varepsilon}=\left(p^{2}+m^{2}\right)^{1 / 2}, \sigma= \pm 1 / 2$ and $e= \pm 1$. The $4 \times 4$ unit matrix can be expanded as

$$
I=\sum_{\sigma, e} \int d \boldsymbol{p}|\boldsymbol{p} \sigma e\rangle\langle\boldsymbol{p} \sigma e| .
$$

Taking into account that

$$
\langle\boldsymbol{r} \mid \boldsymbol{p} \sigma e\rangle=u(\boldsymbol{p} \sigma e) \frac{e^{i \boldsymbol{p} \cdot \boldsymbol{r}}}{(2 \pi)^{3 / 2}}
$$

with

$$
u(\boldsymbol{p} \sigma+)=\left(\frac{\tilde{\varepsilon}+m}{2 \tilde{\varepsilon}}\right)^{1 / 2}\left(\begin{array}{c}
1 \\
\frac{\boldsymbol{\sigma} \cdot \boldsymbol{p}}{\tilde{\varepsilon}+m}
\end{array}\right) \chi_{\sigma}
$$

and

$$
u(p \sigma-)=\left(\frac{\tilde{\varepsilon}+m}{2 \tilde{\varepsilon}}\right)^{1 / 2}\left(\begin{array}{c}
-\frac{\boldsymbol{\sigma} \cdot \boldsymbol{p}}{\tilde{\varepsilon}+m} \\
1
\end{array}\right) \chi_{\sigma},
$$

the Wigner transform of $\hat{t}$ can be written as follows:

$$
\begin{aligned}
t_{\mathrm{w}} & =\int d s e^{-i \boldsymbol{p} \cdot \boldsymbol{s}}\langle\boldsymbol{q}+\boldsymbol{s} / 2|\hat{t}| \boldsymbol{q}-\boldsymbol{s} / 2\rangle \\
& =\sum_{\sigma, e} \int d \boldsymbol{p}^{\prime} \int d \boldsymbol{s} e^{-i \boldsymbol{p} \cdot \boldsymbol{s}}\left\langle\dot{q}+\boldsymbol{s} / 2|\hat{t}| \boldsymbol{p}^{\prime} \sigma e\right\rangle\left\langle\boldsymbol{p}^{\prime} \sigma e \mid \boldsymbol{q}-\boldsymbol{s} / 2\right\rangle \\
& =\sum_{\sigma, e} e \int d \boldsymbol{p}^{\prime} \tilde{\varepsilon}^{\prime} \int d \boldsymbol{s} e^{-i \boldsymbol{p} \cdot \boldsymbol{s}}\left\langle\boldsymbol{q}+\boldsymbol{s} / 2 \mid \boldsymbol{p}^{\prime} \sigma e\right\rangle\left\langle\boldsymbol{p}^{\prime} \sigma e \mid \boldsymbol{q}-\boldsymbol{s} / 2\right\rangle \\
& =\sum_{\sigma, e} e \int d \boldsymbol{p}^{\prime} \tilde{\varepsilon}^{\prime} u\left(\boldsymbol{p}^{\prime} \sigma e\right) u^{\dagger}\left(\boldsymbol{p}^{\prime} \sigma e\right) \frac{1}{(2 \pi)^{3}} \int d \boldsymbol{s} e^{i\left(\boldsymbol{p}^{\prime}-\boldsymbol{p}\right) \cdot \boldsymbol{s}} \\
& =\sum_{\sigma, e} e \int d \boldsymbol{p}^{\prime} \tilde{\varepsilon}^{\prime} u\left(\boldsymbol{p}^{\prime} \sigma e\right) u^{\dagger}\left(\boldsymbol{p}^{\prime} \sigma e\right) \delta\left(\boldsymbol{p}^{\prime}-\boldsymbol{p}\right) \\
& =\tilde{\varepsilon} \sum_{\sigma, e} e u(\boldsymbol{p} \sigma e) u^{\dagger}(\boldsymbol{p} \sigma e) .
\end{aligned}
$$


Upon insertion of (2.32) and (2.33) into (2.34), a simple calculation leads to

$$
t_{\mathrm{w}}=\boldsymbol{\alpha} \cdot \boldsymbol{p}+\beta m,
$$

and thereby we prove our anticipation that

$$
H_{\mathrm{w}}=\boldsymbol{\alpha} \cdot \boldsymbol{p}+\beta m^{*}+I V .
$$

\subsubsection{Wigner transform of the relativistic propagator}

For the hamiltonian (2.36), the lowest order $G_{0}$ of the propagator takes a simple form. As

$$
\begin{aligned}
\left(\alpha \cdot p+\beta m^{*}\right)^{2 k} & =\varepsilon^{2 k} I, \\
\left(\alpha \cdot p+\beta m^{*}\right)^{2 k+1} & =\varepsilon^{2 k}\left(\alpha \cdot p+\beta m^{*}\right), \quad k=0,1,2, \ldots,
\end{aligned}
$$

where $\varepsilon=\left(p^{2}+m^{* 2}\right)^{1 / 2}$ is the dispersion relation of the effective particle, then

$$
\begin{aligned}
G_{0} & =\exp \left(-\eta H_{w}\right) \\
& =\exp \left[-\eta\left(\alpha \cdot p+\beta m^{*}\right)\right] \exp (-\eta I V) \\
& =\left[\sum_{k-\text { even }} \frac{\eta^{k}}{k !} \varepsilon^{k} I-\sum_{k-\text { odd }} \frac{\eta^{k}}{k !} \varepsilon^{k-1}\left(\alpha \cdot p+\beta m^{*}\right)\right] \exp (-\eta V) \\
& =\left[I \cosh \eta \varepsilon-\frac{\sinh \eta \varepsilon}{\varepsilon}\left(\alpha \cdot p+\beta m^{*}\right)\right] \exp (-\eta V) .
\end{aligned}
$$

Since the hyperbolic functions which appear in (2.38) can be written in terms of $\exp (-\eta \varepsilon)$ and $\exp (+\eta \varepsilon)$, it is clearly seen that $G_{0}$ contains the positive and negative energy solutions separately. In addition, all the contributions coming from the expansion of the exponential in Eq. (2.1) in powers of $\eta$ have been consistently resummed. From the structure of Eq. (2.21), it is evident that these two features of $G_{0}$ are kept to any order $G_{n}$ in the $\hbar$ expansion of the propagator. These are the main advantages of our formalism in comparison with other alternatives, as could be the use of the Kirzhnits method or the direct calculation of the Wigner transform of Eq. (2.1).

For further understanding of the significance of the indicated properties of $G_{0}$ and the higher orders $G_{n}$ we may compare, for example, the simple and compact result for 
the trace of $G_{0}$ that one gets from Eq. (2.38)

$$
\operatorname{Tr}\left(G_{0}\right)=2[\exp [-\eta(V+\varepsilon)]+\exp [-\eta(V-\varepsilon)]]
$$

with the infinite series in powers of $\eta$ that the direct Wigner transform of Eq. (2.1) would yield for $G_{0}$, where strong cancellations take place between positive and negative energy solutions:

$$
\begin{aligned}
\operatorname{Tr}\left(G_{0}\right) & =\left.\operatorname{Tr}\left([\exp (-\eta \hat{H})]_{\mathrm{w}}\right)\right|_{\hbar^{0}} \\
& =\left.\operatorname{Tr}\left(I-\eta H_{\mathrm{w}}+\frac{\eta^{2}}{2}\left(\hat{H}^{2}\right)_{\mathrm{w}}-\frac{\eta^{3}}{6}\left(\hat{H}^{3}\right)_{\mathrm{w}}+\ldots\right)\right|_{\hbar^{0}} \\
& =2\left[2-2 \eta V+\eta^{2}\left(\varepsilon^{2}+V^{2}\right)-\frac{\eta^{3}}{3}\left(3 \varepsilon^{2} V+V^{3}\right)+\ldots\right] .
\end{aligned}
$$

Naturally, the result displayed in Eq. (2.40) is nothing else but the Taylor expansion of Eq. (2.39).

It is worth noting that in the situations where $\hat{H}$ does not have a matrix structure, ordinarily there is no term of first order in $\hbar$ in the expansion of the propagator [Ri80]. On the contrary, if the hamiltonian contains matrices which do not commute as in the present case, there appear odd powers of $\hbar$. However, these terms have zero trace and do not contribute to the physical quantities of interest as we shall see later.

Starting from $G_{0}$, the explicit evaluation of $G_{1}$ and $G_{2}$ from Eqs. (2.17) and (2.21) is in principle straightforward for the Dirac hamiltonian (2.23). In practice, it is not an easy task due to the fact that the calculations are very long and the expressions quite lengthy, and one has to be very careful not to make any trivial mistake, especially with the vector algebra of the Pauli matrices. We have developed a REDUCE computer code [He84, Ra87] to handle the hardest job. We do not give here further details of the intermediate steps of the calculations, neither the final expressions of $G_{1}$ and $G_{2}$ which involve the gradients of the fields $V$ and $S$. Instead, we collect in Appendix A the correspondig terms of the semiclassical density matrix, which is closely related to the propagator as we shall describe below. 


\subsubsection{Connection with other approaches}

Before closing this section on the $\hbar$ expansion of the relativistic Bloch propagator, it may be appropiate to say a few words about other ways which have been pursued to perform semiclassical expansions of the relativistic problem. The authors of references [En89, We91, Sp92] start from the equation of motion of the Green's function or Feynman propagator

$$
\left(i \gamma^{0} \partial_{0}-\gamma^{0} \hat{H}\right) G_{\mathrm{F}}\left(x, x^{\prime}\right)=\delta^{4}\left(x-x^{\prime}\right),
$$

where $\hat{H}$ is a time-independent Dirac hamiltonian (notation as in [Bj64]). Expansion of $G_{\mathrm{F}}$ into a formal power series of $\hbar$

$$
G_{\mathrm{F}}\left(x, x^{\prime}\right)=\sum_{n} \hbar^{n} G_{\mathrm{F}}^{(n)}\left(x, x^{\prime}\right),
$$

followed by a Fourier transform of $G_{\mathrm{F}}^{(n)}\left(x, x^{\prime}\right)$ [En89, Sp92] or by a Wigner transform of the Dyson equation [We91], leads to a recursion relation which can be solved iteratively to obtain the $G_{\mathrm{F}}^{(\mathfrak{n})}\left(x, x^{\prime}\right)$. Observables like, e.g., the particle density are then obtained from the well-known identity [Gr85]

$$
\rho(\boldsymbol{r})=-i \lim _{x^{\prime} \rightarrow x} \operatorname{Tr}\left[\gamma^{0} G_{\mathrm{F}}\left(x, x^{\prime}\right)\right] .
$$

The relation of the Green's function with the Bloch propagator (2.1) is rather indirect (stemming from different boundary conditions):

$$
G_{\mathrm{F}}=-i\left[\Theta\left(t-t^{\prime}\right) \Lambda^{+}-\Theta\left(t^{\prime}-t\right) \Lambda^{-}\right] \gamma^{0} \hat{G}\left(i t-i t^{\prime}\right),
$$

where

$$
\Lambda^{ \pm}=\sum_{n}\left|\chi_{n}^{ \pm}\right\rangle\left\langle\chi_{n}^{ \pm}\right|
$$

with $\hat{H}\left|\chi_{n}^{ \pm}\right\rangle=E_{n}^{ \pm}\left|\chi_{n}^{ \pm}\right\rangle$, are the projection operators on positive and negative energy states. Of course, all necessary information is contained in both propagators $G_{\mathrm{F}}$ and $\hat{G}$.

To show that Eq. (2.43) holds, let us recall the expression of the Green's function in terms of the eigenstates and eigenvalues of $\hat{H}$ [Bj64, Gr85]:

$$
\begin{aligned}
i \gamma^{0} G_{\mathrm{F}}= & \Theta\left(t-t^{\prime}\right) \sum_{n}\left|\chi_{n}^{+}\right\rangle\left\langle\chi_{n}^{+}\right| \exp \left[-i E_{n}^{+}\left(t-t^{\prime}\right)\right] \\
& -\Theta\left(t^{\prime}-t\right) \sum_{n}\left|\chi_{n}^{-}\right\rangle\left\langle\chi_{n}^{-}\right| \exp \left[-i E_{n}^{-}\left(t-t^{\prime}\right)\right],
\end{aligned}
$$


which is retarded for positive energy solutions (Dirac particles propagating into the future) and advanced for negative energy solutions (motion backwards in time). Owing to the hermiticity of $\hat{H}$, when we multiply the Bloch propagator $\hat{G}\left(i t-i t^{\prime}\right)$ by $\Lambda^{ \pm}$from the left we obtain

$$
\Lambda^{ \pm} \hat{G}\left(i t-i t^{\prime}\right)=\sum_{n}\left|\chi_{n}^{ \pm}\right\rangle\left\langle\chi_{n}^{ \pm}\right| \exp \left[-i E_{n}^{ \pm}\left(t-t^{\prime}\right)\right]
$$

and, in view of (2.45), Eq. (2.43) follows. We may verify that the matrix elements of Eq. (2.43) in the coordinate representation obey the Dyson equation (2.41). We then start by applying to $G_{\mathrm{F}}\left(x, x^{\prime}\right)$ the time derivative. Using (2.43), this leads to

$$
\begin{aligned}
i \gamma^{0} \partial_{0} G_{F}\left(x, x^{\prime}\right)=\frac{\partial}{\partial t}[ & \Theta\left(t-t^{\prime}\right)\left\langle\boldsymbol{r}\left|\Lambda^{+} \hat{G}\left(i t-i t^{\prime}\right)\right| \boldsymbol{r}^{\prime}\right\rangle \\
& \left.-\Theta\left(t^{\prime}-t\right)\left\langle\boldsymbol{r}\left|\Lambda^{-} \hat{G}\left(i t-i t^{\prime}\right)\right| \boldsymbol{r}^{\prime}\right\rangle\right] .
\end{aligned}
$$

Recognizing that $d \Theta(\tau) / d \tau=\delta(\tau)$ and

$$
\begin{aligned}
\frac{\partial}{\partial t}\left\langle\boldsymbol{r}\left|\Lambda^{ \pm} \hat{G}\left(i t-i t^{\prime}\right)\right| \boldsymbol{r}^{\prime}\right\rangle & =\sum_{n}\left\langle\boldsymbol{r} \mid \chi_{n}^{ \pm}\right\rangle\left\langle\chi_{n}^{ \pm} \mid \boldsymbol{r}^{\prime}\right\rangle \frac{d}{d t} \exp \left[-i E_{n}^{ \pm}\left(t-t^{\prime}\right)\right] \\
& =-i \hat{H}\left\langle\boldsymbol{r}\left|\Lambda^{ \pm} \hat{G}\left(i t-i t^{\prime}\right)\right| \boldsymbol{r}^{\prime}\right\rangle
\end{aligned}
$$

a straightforward calculation yields

$$
\begin{aligned}
i \gamma^{0} \partial_{0} G_{\mathrm{F}}\left(x, x^{\prime}\right)= & \delta\left(t-t^{\prime}\right)\left\langle\boldsymbol{r}\left|\Lambda^{+}+\Lambda^{-}\right| \boldsymbol{r}^{\prime}\right\rangle \\
& -i \hat{H}\left[\Theta\left(t-t^{\prime}\right)\left\langle\boldsymbol{r}\left|\Lambda^{+} \hat{G}\left(i t-i t^{\prime}\right)\right| \boldsymbol{r}^{\prime}\right\rangle\right. \\
& \left.-\Theta\left(t^{\prime}-t\right)\left\langle\boldsymbol{r}\left|\Lambda^{-} \hat{G}\left(i t-i t^{\prime}\right)\right| \boldsymbol{r}^{\prime}\right\rangle\right],
\end{aligned}
$$

which can be brought to the form (2.41) bearing in mind that $\Lambda^{+}+\Lambda^{-}=I$ due to the orthogonality and completeness of the set of eigenstates $\left|\chi_{n}^{ \pm}\right\rangle$.

As a matter of interest, we finally mention that the Fourier transform of the Bloch propagator with respect to the coordinate $\boldsymbol{r}^{\prime}$,

$$
g(\boldsymbol{r}, \boldsymbol{p})=\int d \boldsymbol{r}^{\prime} e^{-\boldsymbol{\imath} \boldsymbol{p} \cdot\left(\boldsymbol{r}-\boldsymbol{r}^{\prime}\right)} \hat{G}\left(\boldsymbol{r}, \boldsymbol{r}^{\prime}\right),
$$


is related to the Wigner transform $G_{\mathrm{w}}(\boldsymbol{q}, \boldsymbol{p})$ simply by [Je78]

$$
G_{\mathrm{w}}(\boldsymbol{q}, \boldsymbol{p})=\exp \left(\frac{i \hbar}{2} \nabla_{\boldsymbol{p}} \cdot \nabla_{q}\right) g(\boldsymbol{q}, \boldsymbol{p})
$$

(cf. Eqs. (2.25)-(2.26) for notation).

On combination with (2.43), Eq. (2.49) could in principle be used to relate the Fourier transform of the Green's function with the Wigner transform of the Bloch propagator and, therefore, to relate the $\hbar$ expansion of Speicher et al. [Sp92] with ours. Despite this charming programme, the exponential in Eq. (2.49) introduces a new power series of $\hbar$ which adds to the others. Thus, there exists no simple relation, order by order, between the coefficients of the expansion of the Fourier transformed Green's function and the Wigner-Kirkwod expansion of the Bloch propagator, as the arrangement of the $\hbar$ contributions in the two series is different.

It seems a general fact that the $\hbar$ expansion comes out more easily in a transformed space than in ordinary coordinate space. The Wigner representation has the interesting feature that it explicitly keeps the symmetry with respect to the interchange of the variables $\boldsymbol{r}$ and $\boldsymbol{r}^{\prime}$.

\subsection{The Relativistic Wigner-Kirkwood Density Matrix}

The generalized density matrix $\hat{\mathcal{R}}$ is obtained from the propagator $\hat{G}$ by an inverse Laplace transform (see e.g. [Ri80]):

$$
\hat{\mathcal{R}}(\lambda)=\frac{1}{2 \pi i} \int_{c-i \infty}^{c+i \infty} d \eta \exp (\eta \lambda) \frac{\hat{G}(\eta)}{\eta}=\mathcal{L}_{\eta \rightarrow \lambda}^{-1}\left[\frac{\hat{G}(\eta)}{\eta}\right]
$$

where $\lambda$ is the chemical potential and we have made explicit the dependence of $\hat{\mathcal{R}}$ and $\hat{G}$ on $\lambda$ and $\eta$, respectively.

Inserting the $\hbar$ expansion of the propagator into (2.50), one finds the expression of the corresponding Wigner-Kirkwood density matrix:

$$
\mathcal{R}_{\mathrm{w}}=\mathcal{L}_{\eta \rightarrow \lambda}^{-1}\left[\frac{G_{\mathrm{w}}(\eta)}{\eta}\right]=\mathcal{R}_{0}+\hbar \mathcal{R}_{1}+\hbar^{2} \mathcal{R}_{2}+\ldots
$$


From the definitions of the Laplace transform of the step function and of the derivative of a Laplace transform [Ab65], one formally gets

$$
\mathcal{L}_{\eta \rightarrow \lambda}^{-1}\left[\eta^{n} \frac{\exp \{-\eta(V \pm \varepsilon)\}}{\eta}\right]=\frac{\partial^{n}}{\partial \lambda^{n}} \Theta(\lambda-V \mp \varepsilon)
$$

Therefore, the $4 \times 4$ relativistic density matrix $\mathcal{R}_{\mathrm{w}}$ will contain the step function, the delta function and its derivatives. As it happens in the non-relativistic problem, $\mathcal{R}_{\mathrm{w}}$ has to be considered as a distribution rather than as a function, in the sense that it is only meaningful if used under an integral sign to compute expectation values of one-body operators. For instance, the zero-order term of the density matrix (cf. Eq. $(2.38))$ is

$$
\begin{aligned}
\mathcal{R}_{0}= & \frac{\Theta\left(\lambda^{+}-V-\varepsilon\right)}{2}\left[I+\frac{1}{\varepsilon}\left(\boldsymbol{\alpha} \cdot \boldsymbol{p}+\beta m^{*}\right)\right] \\
+ & \frac{\Theta\left(\lambda^{-}-V+\varepsilon\right)}{2}\left[I-\frac{1}{\varepsilon}\left(\boldsymbol{\alpha} \cdot \boldsymbol{p}+\beta m^{*}\right)\right],
\end{aligned}
$$

where the first line corresponds to the positive energy states and the second line to the negative ones. We also indicate that the chemical potentials $\left(\lambda^{+}, \lambda^{-}\right)$may be different in the two cases. Using Eqs. (2.51) and (2.52), $\mathcal{R}_{1}$ and $\mathcal{R}_{2}$ are straightforwardly obtained from $G_{1}$ and $G_{2}$, respectively. We collect their expressions in Appendix A.

As we have shown for the propagator, the positive and negative energy states are separated in the expression of the density matrix, and the infinite series in powers of $\eta$ has been automatically summed to each order in $\hbar$. On the other hand, a very interesting property of the density matrix $\hat{\mathcal{R}}$ is its idempotency [Ri80], which holds for the positive $\left(\hat{\mathcal{R}}^{+}\right)$, and negative $\left(\hat{\mathcal{R}}^{-}\right)$energy solutions ${ }^{\dagger}$ separately:

$$
\left(\hat{\mathcal{R}}^{ \pm}\right)^{2}=\hat{\mathcal{R}}^{ \pm}
$$

Let us take the Wigner transform of this equation, apply Eq. (2.7) to its 1.h.s. and insert the $\hbar$ expansion Eq. (2.51) of the density matrix. Regrouping the terms of the same order in $\hbar$, we then find a set of relationships which must be fulfilled by each order of the semiclassical expansion of the density matrix, independently for positive

tObserve that although the density matrix $\hat{\mathcal{R}}^{ \pm}$and the projection operators $\Lambda^{ \pm}$introduced in Eq. (2.44) are closely related, they should not be confused since $\hat{\mathcal{R}}^{ \pm}=\sum_{n}\left|\chi_{n}^{ \pm}\right\rangle\left\langle\chi_{n}^{ \pm}\right| \Theta\left(\lambda^{ \pm}-E_{n}^{ \pm}\right)$. 
and negative energy solutions. Eq. (2.54) yields the following three relations up to order $\hbar^{2}$ :

$$
\begin{aligned}
& \mathcal{R}_{0}^{ \pm}=\mathcal{R}_{0}^{ \pm 2}, \\
& \mathcal{R}_{1}^{ \pm}=\left\{\mathcal{R}_{0}^{ \pm}, \mathcal{R}_{1}^{ \pm}\right\}+\frac{i}{2} \mathcal{R}_{0}^{ \pm} \overleftrightarrow{\Lambda} \mathcal{R}_{0}^{ \pm}, \\
& \mathcal{R}_{2}^{ \pm}=\mathcal{R}_{1}^{ \pm 2}+\left\{\mathcal{R}_{0}^{ \pm}, \mathcal{R}_{2}^{ \pm}\right\}+\frac{i}{2}\left\{\mathcal{R}_{0}^{ \pm}, \overleftrightarrow{\Lambda}, \mathcal{R}_{1}^{ \pm}\right\}-\frac{1}{8} \mathcal{R}_{0}^{ \pm} \overleftrightarrow{\Lambda}^{2} \mathcal{R}_{0}^{ \pm} .
\end{aligned}
$$

Eq. (2.55) constitutes a stringent test of the correctness of the calculated semiclassical density matrix.

We have checked that the results we have found for the relativistic $\hbar^{2}$-order $\mathcal{R}_{\mathrm{w}}$ matrix satisfy (2.55). In the case of the Dirac hamiltonian, this is an extremely lengthy proof and we do not present it here (again the calculations were performed with REDUCE software). Nevertheless, we think it is worth showing some interesting ingredients of the procedure. We do so in Appendix B where we prove that the well-known WK expansion of the local density matrix to order $\hbar^{2}$ corresponding to a non-relativistic hamiltonian [Je78] fulfils (2.55).

\subsection{Semiclassical Relativistic Particle and Energy Densities}

Let us now apply the semiclassical relativistic density matrix we have obtained in the preceding section to calculate the $\hbar$ expansion of the particle and energy densities, in both the WK approach and the energy density (RETF) formalism.

For a given single-particle operator $\hat{O}$, we define its expectation value as

$$
\langle\hat{O}\rangle=\frac{1}{(2 \pi)^{3}} \int d \boldsymbol{r} \int d \boldsymbol{p} \operatorname{Tr}^{+}[\hat{O}(\boldsymbol{r}, \hat{\boldsymbol{p}}) \hat{\mathcal{R}}(\boldsymbol{r}, \hat{\boldsymbol{p}})]_{\mathrm{w}},
$$

where $\mathrm{Tr}^{+}$means that the trace is taken disregarding the negative energy terms. Thus, here and in the following we only consider the positive energy solutions, i.e., we restrict ourselves to the positive energy part of the spectrum and neglect any contribution from antiparticles, as it is currently done in most applications of relativistic mean field theory. In the case of the operators $\hat{O}$ we will be considering in this section, the semiclassical 
expectation value corresponding to Eq. (2.56) reduces to

$$
\langle\hat{O}\rangle_{\mathrm{sc}}=\frac{1}{(2 \pi)^{3}} \int d \boldsymbol{r} \int d \boldsymbol{p} \operatorname{Tr}^{+}\left[O_{\mathrm{w}}\left(\mathcal{R}_{0}+\hbar^{2} \mathcal{R}_{2}\right)\right]
$$

up to order $\hbar^{2}$, since the other terms which in principle could contribute turn out to be zero or vanish after angular average in momentum space. Indeed, this fact was expected for the contributions of first order in $\hbar$, as we are within a static approximation and the nuclear ground-state is even under time reversal.

\subsubsection{Wigner-Kirkwood expressions}

Some details relevant to the calculation of the integrals over $p$ in (2.57) are treated in Appendix C. Assuming that the integrands are invariant under rotations in momentum space, and introducing the local Fermi momentum

$$
k_{\mathrm{F}}=\left[(\lambda-V)^{2}-m^{* 2}\right]^{1 / 2}
$$

and the definitions

$$
\begin{aligned}
& \varepsilon_{\mathrm{F}}=\lambda-V=\left(k_{\mathrm{F}}^{2}+m^{* 2}\right)^{1 / 2} \\
& x_{\mathrm{F}}=\varepsilon_{\mathrm{F}} / k_{\mathrm{F}}
\end{aligned}
$$

the WK particle $\rho(\boldsymbol{r})$, scalar $\rho_{\mathrm{s}}(\boldsymbol{r})$, energy $\mathcal{E}(\boldsymbol{r})$, and kinetic energy $\tau(\boldsymbol{r})$ densities to order $\hbar^{2}$ for each kind of nucleons are found to be:

$$
\begin{aligned}
\rho(\boldsymbol{r})= & \frac{1}{(2 \pi)^{3}} \int d \boldsymbol{p} \operatorname{Tr}^{+}[\hat{\mathcal{R}}(\boldsymbol{r}, \hat{\boldsymbol{p}})]_{\mathrm{w}}=\rho_{0}+\rho_{2}, \\
\rho_{0}= & \frac{k_{\mathrm{F}}^{3}}{3 \pi^{2}}, \\
\rho_{2}= & \frac{1}{24 \pi^{2}}\left[\frac{1}{k_{\mathrm{F}}}\left(3-x_{\mathrm{F}}^{2}\right)(\nabla V)^{2}-\left(2 x_{\mathrm{F}}+4 \ln \frac{k_{\mathrm{F}}+\varepsilon_{\mathrm{F}}}{m^{*}}\right) \Delta V\right. \\
& +2 \frac{x_{\mathrm{F}}}{m^{*}}\left(3-x_{\mathrm{F}}^{2}\right)\left(\nabla V \cdot \nabla m^{*}\right)+\frac{1}{k_{\mathrm{F}}}\left(2-x_{\mathrm{F}}^{2}\right)\left(\nabla m^{*}\right)^{2} \\
& \left.+2 \frac{k_{\mathrm{F}}}{m^{*}}\left(1-x_{\mathrm{F}}^{2}\right) \Delta m^{*}\right]
\end{aligned}
$$




$$
\begin{aligned}
& \rho_{\mathrm{s}}(\boldsymbol{r})=\frac{1}{(2 \pi)^{3}} \int d \boldsymbol{p} \operatorname{Tr}^{+}[\beta \hat{\mathcal{R}}(\boldsymbol{r}, \hat{\boldsymbol{p}})]_{\mathrm{w}}=\rho_{\mathrm{s}, 0}+\rho_{\mathrm{s}, 2}, \\
& \rho_{\mathrm{s}, 0}=\frac{m^{*}}{2 \pi^{2}}\left[k_{\mathrm{F}} \varepsilon_{\mathrm{F}}-m^{* 2} \ln \frac{k_{\mathrm{F}}+\varepsilon_{\mathrm{F}}}{m^{*}}\right] \text {, } \\
& \rho_{s, 2}=-\frac{1}{24 \pi^{2}}\left[\frac{x_{F}}{m^{*}}\left(1+x_{\mathrm{F}}^{2}\right)(\nabla V)^{2}+2 \frac{m^{*}}{k_{\mathrm{F}}} \Delta V\right. \\
& +\frac{2}{k_{\mathrm{F}}}\left(2+x_{\mathrm{F}}^{2}\right)\left(\nabla V \cdot \nabla m^{*}\right)+\frac{x_{\mathrm{F}}}{m^{*}}\left(2+x_{\mathrm{F}}^{2}\right)\left(\nabla m^{*}\right)^{2} \\
& \left.+\left(2 x_{\mathrm{F}}-6 \ln \frac{k_{\mathrm{F}}+\varepsilon_{\mathrm{F}}}{m^{*}}\right) \Delta m^{*}\right] \\
& \mathcal{E}(\boldsymbol{r})=\frac{1}{(2 \pi)^{3}} \int d \boldsymbol{p} \operatorname{Tr}^{+}\left[\left(\boldsymbol{\alpha} \cdot \hat{\boldsymbol{p}}+\beta m^{*}(\boldsymbol{r})+I V(\boldsymbol{r})\right) \hat{\mathcal{R}}(\boldsymbol{r}, \hat{\boldsymbol{p}})\right]_{\mathrm{w}}=\mathcal{E}_{0}+\mathcal{E}_{2} \\
& \mathcal{E}_{0}=\frac{1}{8 \pi^{2}}\left[k_{\mathrm{F}} \varepsilon_{\mathrm{F}}^{3}+k_{\mathrm{F}}^{3} \varepsilon_{\mathrm{F}}-m^{* 4} \ln \frac{k_{\mathrm{F}}+\varepsilon_{\mathrm{F}}}{m^{*}}\right]+V \rho_{0}, \\
& \mathcal{E}_{2}=\frac{1}{24 \pi^{2}}\left[\left(x_{\mathrm{F}}\left(2-x_{\mathrm{F}}^{2}\right)-2 \ln \frac{k_{\mathrm{F}}+\varepsilon_{\mathrm{F}}}{m^{*}}\right)(\nabla V)^{2}\right. \\
& -2 k_{\mathrm{F}}\left(1+x_{\mathrm{F}}^{2}\right) \Delta V+2 \frac{m^{*}}{k_{\mathrm{F}}}\left(1-x_{\mathrm{F}}^{2}\right)\left(\nabla V \cdot \nabla m^{*}\right) \\
& +\left(x_{\mathrm{F}}\left(1-x_{\mathrm{F}}^{2}\right)-\ln \frac{k_{\mathrm{F}}+\varepsilon_{\mathrm{F}}}{m^{*}}\right)\left(\nabla m^{*}\right)^{2} \\
& \left.-2 m^{*}\left(x_{\mathrm{F}}-\ln \frac{k_{\mathrm{F}}+\varepsilon_{\mathrm{F}}}{m^{*}}\right) \Delta m^{*}\right]+V \rho_{2}
\end{aligned}
$$

and finally

$$
\begin{aligned}
\tau(\boldsymbol{r})= & \frac{1}{(2 \pi)^{3}} \int d \boldsymbol{p} \operatorname{Tr}^{+}[(\boldsymbol{\alpha} \cdot \hat{\boldsymbol{p}}+\beta m-I m) \hat{\mathcal{R}}(\boldsymbol{r}, \hat{\boldsymbol{p}})]_{\mathrm{w}}=\tau_{0}+\tau_{2}, \\
\tau_{0}= & \frac{1}{8 \pi^{2}}\left[2 k_{\mathrm{F}} \varepsilon_{\mathrm{F}}^{3}-m^{* 2}\left(5-4 \frac{m}{m^{*}}\right) k_{\mathrm{F}} \varepsilon_{\mathrm{F}}+m^{* 4}\left(3-4 \frac{m}{m^{*}}\right) \ln \frac{k_{\mathrm{F}}+\varepsilon_{\mathrm{F}}}{m^{*}}\right] \\
& -m \frac{k_{\mathrm{F}}^{3}}{3 \pi^{2}}
\end{aligned}
$$




$$
\begin{aligned}
\tau_{2}=\frac{1}{24 \pi^{2}}\{[ & \left.\left(3-\left(1+x_{\mathrm{F}}^{2}\right) \frac{m}{m^{*}}\right) x_{\mathrm{F}}-\left(3-x_{\mathrm{F}}^{2}\right) \frac{m}{k_{\mathrm{F}}}-2 \ln \frac{k_{\mathrm{F}}+\varepsilon_{\mathrm{F}}}{m^{*}}\right](\nabla V)^{2} \\
& +2 m\left[x_{\mathrm{F}}-\frac{k_{\mathrm{F}}}{m}\left(2-\left(1-x_{\mathrm{F}}^{2}\right) \frac{m}{m^{*}}\right)+2 \ln \frac{k_{\mathrm{F}}+\varepsilon_{\mathrm{F}}}{m^{*}}\right] \Delta V \\
& -\left[2 \frac{m}{k_{\mathrm{F}}}\left(2+x_{\mathrm{F}}^{2}\right)+2 \frac{m}{m^{*}} x_{\mathrm{F}}\left(3-x_{\mathrm{F}}^{2}\right)+6 \frac{k_{\mathrm{F}}}{m^{*}}\left(1-x_{\mathrm{F}}^{2}\right)\right]\left(\nabla V \cdot \nabla m^{*}\right) \\
& -\left[\frac{m}{k_{\mathrm{F}}}\left(2-x_{\mathrm{F}}^{2}\right)-\left(3-2 \frac{m}{m^{*}}\right) x_{\mathrm{F}}+\frac{m}{m^{*}} x_{\mathrm{F}}^{3}+\ln \frac{k_{\mathrm{F}}+\varepsilon_{\mathrm{F}}}{m^{*}}\right]\left(\nabla m^{*}\right)^{2} \\
& \left.-2 m\left[x_{\mathrm{F}}+\frac{k_{\mathrm{F}}}{m^{*}}\left(1-x_{\mathrm{F}}^{2}\right)-\left(3-2 \frac{m^{*}}{m}\right) \ln \frac{k_{\mathrm{F}}+\varepsilon_{\mathrm{F}}}{m^{*}}\right] \Delta m^{*}\right\} \cdot(2.71)
\end{aligned}
$$

Eqs. (2.61), (2.64), (2.67) and (2.70) correspond to the $\hbar^{0}$-order WK approach, whereas Eqs. (2.62), (2.65), (2.68) and (2.71) are the respective $\hbar^{2}$-order WK corrections.

Parenthetically it is worthwhile indicating that if we were dealing with applications where the self-energy operator is determined from a many-body calculation, a term

$$
\frac{1}{2}[\beta S(r)+I V(r)]
$$

should be subtracted from the hamiltonian to avoid double counting of the meson contributions to the energy. In that case the energy density would become

$$
\varepsilon-\frac{1}{2}\left(m^{*}-m\right) \rho_{\mathbf{s}}-\frac{1}{2} V \rho=\frac{1}{2}(\mathcal{E}+\tau+m \rho) .
$$

In mean field approximation, the expressions obtained by Speicher, Dreizler and Engel [Sp92] using the Green's function formalism and by Von-Eiff, Haddad and Weigel [Ei91b] from the Wigner transformed Dyson equation are identical to ours. Setting $m^{*}=m$ one recovers the expressions already known in the atomic case [En87]. We have also looked for the non-relativistic limit of the particle and kinetic energy densities. One has to expand the above equations in powers of $k_{F} / m^{*}$, retain the lowest orders and finally expand $\left(m^{*}\right)^{n}=m^{n}(1+S / m)^{n}$ in terms of $S / m$. This yields the standard non-relativistic formulae for $\rho$ and $\tau$ [Ri80] for a set of fermions moving in a potential well, which in this case is the sum $V+S$ of the meson potentials. In this limit the scalar and particle densities coincide, $\rho_{\mathbf{s}}=\rho$, as the small component of the wave function is neglected. 


\subsubsection{Density functionals}

To obtain the RETF density functionals we have to write $\nabla V$ and $\Delta V$ as a function of the particle density, the effective mass and their gradients, and insert them into the $\hbar^{2}$ terms of the WK expansions. That is, we have to eliminate $V$ in favour of $\rho$ and $m^{*}$ in Eqs. (2.65), (2.68) and (2.71). For that purpose, one has to invert the semiclassical expansion of the particle density. This is achieved first by putting in Eq. (2.61) the expression (2.58) of $k_{\mathrm{F}}$ in terms of $V$

$$
\rho_{0}=\frac{1}{3 \pi^{2}}\left[(\lambda-V)^{2}-m^{* 2}\right]^{3 / 2},
$$

and computing then $\nabla V$ and $\Delta V$ from this equation. This results in

$$
\begin{aligned}
\nabla V= & -\frac{\pi^{2}}{k_{\mathrm{F}} \varepsilon_{\mathrm{F}}} \nabla \rho_{0}-\frac{m^{*}}{\varepsilon_{\mathrm{F}}} \nabla m^{*}, \\
\Delta V= & \frac{\pi^{2}\left(k_{\mathrm{F}}^{2}+\varepsilon_{\mathrm{F}}^{2}\right)}{3 k_{\mathrm{F}} \varepsilon_{\mathrm{F}}^{3}} \frac{\left(\nabla \rho_{0}\right)^{2}}{\rho_{0}}-\frac{\pi^{2}}{k_{\mathrm{F}} \varepsilon_{\mathrm{F}}} \Delta \rho_{0}+\frac{2 \pi^{2} m^{*}}{k_{\mathrm{F}} \varepsilon_{\mathrm{F}}^{3}}\left(\nabla \rho_{0} \cdot \nabla m^{*}\right) \\
& -\frac{k_{\mathrm{F}}^{2}}{\varepsilon_{\mathrm{F}}^{3}}\left(\nabla m^{*}\right)^{2}-\frac{m^{*}}{\varepsilon_{\mathrm{F}}} \Delta m^{*} .
\end{aligned}
$$

In the next step, as one works with the density $\rho$ rather than with $\rho_{0}$, it is needed to expand $\rho$ around a new variable $k_{0}$ up to order $\hbar^{2}$ :

$$
\rho\left(k_{\mathrm{F}}\right)=\rho_{0}\left(k_{0}\right)+\left(\frac{\partial \rho_{0}\left(k_{0}\right)}{\partial k_{0}}\right) \delta k+\rho_{2}\left(k_{0}\right)+\mathcal{O}\left(\hbar^{4}\right),
$$

where $\delta k=k_{\mathrm{F}}-k_{0}$ and $\rho_{2}$ is defined in (2.62). We then demand that $\rho_{0}\left(k_{0}\right)$ reproduces the exact density:

$$
\rho_{0}\left(k_{0}\right)=\rho\left(k_{\mathrm{F}}\right),
$$

whence it follows that

$$
\begin{aligned}
k_{\mathrm{F}} & =k_{0}-\left[\frac{\partial \rho_{0}\left(k_{0}\right)}{\partial k_{0}}\right]^{-1} \rho_{2}\left(k_{0}\right) \\
& =k_{0}\left(1-\frac{\pi^{2}}{k_{0}^{3}} \rho_{2}\left(k_{0}\right)\right)
\end{aligned}
$$


to order $\hbar^{2}$. Then one substitutes in Eqs. (2.63)-(2.71) for $k_{\mathrm{F}}$ as given by (2.78), and expands these equations around the value $k_{0}$ consistently to order $\hbar^{2}$. Finally, one replaces $k_{0}$ by $k_{\mathrm{F}}$ and takes $k_{\mathrm{F}}=\left(3 \pi^{2} \rho\right)^{1 / 3}$. In this way, we get the scalar, energy and kinetic energy density functionals in the RETF formalism including $\hbar^{2}$-order corrections:

$$
\begin{aligned}
\rho_{\mathbf{s}}(\boldsymbol{r})=\frac{m^{*}}{2 \pi^{2}}\left[k_{\mathrm{F}}\right. & \left.\varepsilon_{\mathrm{F}}-m^{* 2} \ln \frac{k_{\mathrm{F}}+\varepsilon_{\mathrm{F}}}{m^{*}}\right] \\
-\frac{1}{72 \varepsilon_{\mathrm{F}}^{2}}[ & \left(\frac{\varepsilon_{\mathrm{F}}}{m^{*}}\left(2+3 \frac{m^{* 2}}{\varepsilon_{\mathrm{F}}^{2}}\right)-4 \frac{m^{*}}{k_{\mathrm{F}}}\left(2-\frac{m^{* 2}}{\varepsilon_{\mathrm{F}}^{2}}\right) \ln \frac{k_{\mathrm{F}}+\varepsilon_{\mathrm{F}}}{m^{*}}\right) \frac{(\nabla \rho)^{2}}{\rho} \\
& +12 \frac{m^{*}}{k_{\mathrm{F}}} \ln \frac{k_{\mathrm{F}}+\varepsilon_{\mathrm{F}}}{m^{*}} \Delta \rho \\
& -\frac{6}{k_{\mathrm{F}}}\left(3 \frac{k_{\mathrm{F}}}{\varepsilon_{\mathrm{F}}}+4 \frac{m^{* 2}}{\varepsilon_{\mathrm{F}}^{2}} \ln \frac{k_{\mathrm{F}}+\varepsilon_{\mathrm{F}}}{m^{*}}\right)\left(\nabla \rho \cdot \nabla m^{*}\right) \\
& +\frac{9}{m^{*} k_{\mathrm{F}}}\left(3 \frac{k_{\mathrm{F}}}{\varepsilon_{\mathrm{F}}}+4 \frac{m^{* 2}}{\varepsilon_{\mathrm{F}}^{2}} \ln \frac{k_{\mathrm{F}}+\varepsilon_{\mathrm{F}}}{m^{*}}\right) \rho\left(\nabla m^{*}\right)^{2} \\
& \left.+\frac{18}{k_{\mathrm{F}}}\left(\frac{\varepsilon_{\mathrm{F}}}{k_{\mathrm{F}}}-\left(2+\frac{\varepsilon_{\mathrm{F}}^{2}}{k_{\mathrm{F}}^{2}}\right) \ln \frac{k_{\mathrm{F}}+\varepsilon_{\mathrm{F}}}{m^{*}}\right) \rho \Delta m^{*}\right] \\
\mathcal{E}(\boldsymbol{r})=\frac{1}{8 \pi^{2}} & {\left[k_{\mathrm{F}} \varepsilon_{\mathrm{F}}^{3}+k_{\mathrm{F}}^{3} \varepsilon_{\mathrm{F}}-m^{* 4} \ln \frac{k_{\mathrm{F}}+\varepsilon_{\mathrm{F}}}{m^{*}}\right] } \\
+ & \frac{1}{72 \varepsilon_{\mathrm{F}}^{2}}\left[\left(\varepsilon_{\mathrm{F}}+2 k_{\mathrm{F}} \ln \frac{k_{\mathrm{F}}+\varepsilon_{\mathrm{F}}}{m^{*}}\right) \frac{(\nabla \rho)^{2}}{\rho}\right. \\
+ & +12 \frac{m^{*}}{k_{\mathrm{F}}} \ln \frac{k_{\mathrm{F}}+\varepsilon_{\mathrm{F}}}{m^{*}}\left(\nabla \rho \cdot \nabla m^{*}\right) \\
\left.+\frac{9}{k_{\mathrm{F}}}\left(\frac{\varepsilon_{\mathrm{F}}}{k_{\mathrm{F}}}-\left(2+\frac{\varepsilon_{\mathrm{F}}^{2}}{k_{\mathrm{F}}^{2}}\right) \ln \frac{k_{\mathrm{F}}+\varepsilon_{\mathrm{F}}}{m^{*}}\right) \rho\left(\nabla m^{*}\right)^{2}\right] & \\
&
\end{aligned}
$$


and

$$
\begin{aligned}
\tau(\boldsymbol{r})=\frac{1}{8 \pi^{2}}\left[2 k_{\mathrm{F}} \varepsilon_{\mathrm{F}}^{3}-m^{* 2}\left(5-4 \frac{m}{m^{*}}\right) k_{\mathrm{F}} \varepsilon_{\mathrm{F}}+m^{* 4}\left(3-4 \frac{m}{m^{*}}\right) \ln \frac{k_{\mathrm{F}}+\varepsilon_{\mathrm{F}}}{m^{*}}\right] & +\frac{1}{72 \varepsilon_{\mathrm{F}}^{2}}\left\{\left[\varepsilon_{\mathrm{F}}+\varepsilon_{\mathrm{F}}\left(1-\frac{m}{m^{*}}\right)\left(2-\frac{m^{* 2}}{\varepsilon_{\mathrm{F}}^{2}}\right)\right.\right. \\
& \left.+2 k_{\mathrm{F}}\left(1+2\left(1-\frac{m}{m^{*}}\right) \frac{m^{* 2}}{\varepsilon_{\mathrm{F}}^{2}}\right) \ln \frac{k_{\mathrm{F}}+\varepsilon_{\mathrm{F}}}{m^{*}}\right] \frac{(\nabla \rho)^{2}}{\rho} \\
+ & 12 \frac{m^{*}}{k_{\mathrm{F}}}\left(1-\frac{m}{m^{*}}\right)\left[\frac{k_{\mathrm{F}}}{\varepsilon_{\mathrm{F}}}-\left(1-2 \frac{m^{* 2}}{\varepsilon_{\mathrm{F}}^{2}}\right) \ln \frac{k_{\mathrm{F}}+\varepsilon_{\mathrm{F}}}{m^{*}}\right]\left(\nabla \rho \cdot \nabla m^{*}\right) \\
& -\frac{9}{k_{\mathrm{F}}}\left[\frac{\varepsilon_{\mathrm{F}}}{k_{\mathrm{F}}}+3\left(1-\frac{m}{m^{*}}\right) \frac{k_{\mathrm{F}}}{\varepsilon_{\mathrm{F}}}\right. \\
- & \left.\left.-\left(2+\frac{\varepsilon_{\mathrm{F}}^{2}}{k_{\mathrm{F}}^{2}}-4\left(1-\frac{m}{m^{*}}\right) \frac{m^{* 2}}{\varepsilon_{\mathrm{F}}^{2}}\right) \ln \frac{k_{\mathrm{F}}+\varepsilon_{\mathrm{F}}}{m^{*}}\right] \rho\left(\nabla m^{*}\right)^{2}\right\} \\
-m \rho . & (2.81)
\end{aligned}
$$

We notice that Eqs. (2.80) and (2.81) take this form after suitable partial integration of the terms with $\Delta \rho$ and $\Delta m^{*}$, which assumes that $\rho(\boldsymbol{r})$ vanishes on the surface of a large sphere.

If one takes $m^{*}=m$, Eq. (2.81) reduces to the result of atomic physics [En87]. In the non-relativistic limit $\left(k_{\mathrm{F}} \ll m, S \ll m\right)$ we recover from (2.81) the well-known result

$$
\tau=\tau_{0}+\tau_{2}=\frac{3}{10 m}\left(3 \pi^{2}\right)^{2 / 3} \rho^{5 / 3}+\frac{1}{72 m} \frac{(\nabla \rho)^{2}}{\rho} .
$$

In fact, taking the limit before the elimination of the laplacians in (2.81) by partial integration, the usual term $\Delta \rho / 6 m$ is also found.

Strictly speaking, the density functionals should have been written in terms of the densities $\rho$ and $\rho_{\mathrm{s}}$, the latter instead of $m^{*}$. Obviously, the complicated relation between $\rho_{\mathbf{s}}$ and $m^{*}$, even at order $\hbar^{0}$, does not allow expressions which are analytical. We want to point out that the inversion scheme we have used to derive the density functionals from the WK expansions is not the only possibility which can be considered. An alternative elimination arises if an additional inversion of the effective mass at order $\hbar^{0}$ is also performed [Sp92]. However, in the scheme we have adopted the effective mass is treated in a parallel way to the one which has been traditionally used in the 
non-relativistic case, where no supplementary inversion of $m^{*}$ is made (see e.g. [Gr79] and $[\mathrm{Ba} 85])$. We shall not dwell here any further on this point; we shall come back to it when we study the $\sigma-\omega$ model in Chapter 4. It will be shown there that the variational Euler-Lagrange equations obtained from both inversion schemes turn out to be equivalent.

In concluding this chapter, we note that the semiclassical expansions constructed here are asymptotical by nature and, a priori, are not expected to converge. Nevertheless, one trusts that such expansions truncated after a first few terms provide reliable and accurate enough numerical results, as it happens in the non-relativistic situation. The applications investigated in the next chapters will show that this is also the case in the relativistic context. 


\section{Chapter 3}

\section{Test Calculations with a Relativistic Harmonic Oscillator}

THE HARMONIC oscillator potential has been extensively studied and has found many applications in non-relativistic nuclear physics. We shall use a relativistic harmonic oscillator to investigate quantitatively the accuracy of the semiclassical approximations deduced in the preceding chapter. We specifically consider the situation where the scalar field and the zero component of the vector field correspond to a three-dimensional spherically symmetric harmonic oscillator and have the same strength:

$$
S(r)=V(r)=\frac{1}{2} K r^{2}
$$

In this case the Dirac equation can be solved analytically and simple expressions are obtained for the energy eigenvalues and the wave functions [Sm71, Te90] (other types of a Dirac equation with an oscillator potential which admit analytical solutions are found in the literature [Ku91b]). In this chapter we shall discuss semiclassical WK and RETF results for an ensemble of independent particles submitted to the potential (3.1), in comparison with the quantal results and also with the corresponding Strutinsky averaged calculation. To that end, we begin by solving the Dirac equation. 


\subsection{Solution of the Dirac Equation}

If we write down the Dirac spinor as

$$
\psi=\left(\begin{array}{c}
\varphi_{\mathrm{u}} \\
\varphi_{\mathrm{d}}
\end{array}\right) e^{-i E t},
$$

the Dirac equation associated with the static scalar and vector potentials (3.1) for the large $\varphi_{\mathrm{u}}$ and small $\varphi_{\mathrm{d}}$ components reads

$$
\begin{aligned}
(m+2 V) \varphi_{\mathrm{u}}+(\sigma \cdot \hat{p}) \varphi_{\mathrm{d}} & =E \varphi_{\mathrm{u}} \\
(\sigma \cdot \hat{p}) \varphi_{\mathrm{u}}-m \varphi_{\mathrm{d}} & =E \varphi_{\mathrm{d}} .
\end{aligned}
$$

Combining (3.3) and (3.4), the Dirac equation transforms into a Schrödinger-like equation for the large component $\varphi_{u}$ with an energy-dependent potential [Sm71, Te90]

$$
\left(\frac{\hat{p}^{2}}{2 m}+\frac{E+m}{m} V\right) \varphi_{\mathbf{u}}=\frac{E^{2}-m^{2}}{2 m} \varphi_{\mathbf{u}}
$$

and the small component $\varphi_{\mathrm{d}}$ is obtained from $\varphi_{\mathrm{u}}$ through (3.4),

$$
\varphi_{\mathrm{d}}=\frac{1}{E+m}(\sigma \cdot \hat{p}) \varphi_{\mathrm{u}} .
$$

Unlike Ref. [Te90], we solve this problem without neglecting the mass of the particle. The Dirac spinor (3.2) is rewritten as

$$
\psi_{n l j m}=\left(\begin{array}{c}
i g_{n l} / r \\
f_{n l} / r^{2}(\sigma \cdot r)
\end{array}\right) \varphi_{l j m} e^{-i E_{n l} t}
$$

where

$$
\varphi_{l j m}=\sum_{m_{l}, m_{\mathbf{l}}}\left\langle l \frac{1}{2} m_{l} m_{\mathbf{s}} \mid j m\right\rangle Y_{l, m_{l}}(\theta, \varphi) \chi_{1 / 2, m_{\mathbf{l}}}
$$

and the normalization is

$$
\int_{0}^{\infty} d r\left(g^{2}+f^{2}\right)=1
$$

We have taken advantage of the fact that in a central field the angular part of the twocomponent spinors $\varphi_{\mathrm{u}}$ and $\varphi_{\mathrm{d}}$ are eigenfunctions of the orbital angular momentum, of the spin operators and have definite parity. Note that, since the operator $\sigma \cdot \hat{p}$ changes 
parity, Eqs. (3.3) and (3.4) show that $\varphi_{\mathrm{u}}$ and $\varphi_{\mathrm{d}}$ must have opposite parity. This is why the pseudoscalar $\sigma \cdot r$ is introduced in the definition of $\varphi_{\mathrm{d}}$ in (3.7).

It is easy to show by standard techniques that the physical solution to Eq. (3.5) is

$$
g_{n l}(r)=N_{n l} r\left(\frac{r}{r_{0}}\right)^{l} \exp \left(-r^{2} / 2 r_{0}^{2}\right) L_{n-1}^{l+1 / 2}\left(\frac{r^{2}}{r_{0}^{2}}\right),
$$

where $L_{n}^{\alpha}(x)$ are the generalized Laguerre polynomials [Ab65]. With the assumption (3.7), Eq. (3.6) reads (e.g. [Bj64], ch. 4)

$$
f=\frac{1}{E+m}\left(\frac{d g}{d r}+\kappa \frac{g}{r}\right)
$$

with $\kappa=(2 j+1)(l-j)$. Evaluation of (3.11) from (3.10) yields

$$
f_{n l}(r)=-\frac{N_{n l}}{E_{n l}+m}\left(\frac{r}{r_{0}}\right)^{l+2} \exp \left(-r^{2} / 2 r_{0}^{2}\right)\left[L_{n-1}^{l+3 / 2}\left(\frac{r^{2}}{r_{0}^{2}}\right)+L_{n-2}^{l+3 / 2}\left(\frac{r^{2}}{r_{0}^{2}}\right)\right]
$$

for $j=l+\frac{1}{2}$, and

$$
\begin{aligned}
& f_{n l}(r)= \\
& \quad \frac{N_{n l}}{E_{n l}+m}\left(\frac{r}{r_{0}}\right)^{l} \exp \left(-r^{2} / 2 r_{0}^{2}\right)\left[\left(n+l-\frac{1}{2}\right) L_{n-1}^{l-1 / 2}\left(\frac{r^{2}}{r_{0}^{2}}\right)+n L_{n}^{l-1 / 2}\left(\frac{r^{2}}{r_{0}^{2}}\right)\right]
\end{aligned}
$$

for $j=l-\frac{1}{2}$. The normalization factor is

$$
N_{n l}=\frac{1}{r_{0}}\left[\frac{4(n-1) !\left(E_{n l}+m\right)}{r_{0} \Gamma\left(n+l+\frac{1}{2}\right)\left(3 E_{n l}+m\right)}\right]^{1 / 2} .
$$

The energy eigenvalue is obtained from

$$
\left(E_{n l}-m\right)\left(E_{n l}^{2}-m^{2}\right)=4 K\left(2 n+l-\frac{1}{2}\right)^{2}
$$

and the scale parameter $r_{0}$ is

$$
r_{0}=\frac{1}{\left[K\left(E_{n l}+m\right)\right]^{1 / 4}}
$$

with $n=1,2,3, \ldots, l=0,1,2, \ldots$. Note that $E$ is independent of $j$ (the spin-orbit couplings arising from the scalar field and from the vector field cancel each other) and that $r_{0}$ depends on the state $(n, l)$. A given oscillator shell can be characterized by a 
quantum number $N$ such that $N=2 n+l-2$. In the ultrarelativistic limit, $E_{N} \gg m$, we recover the result of [Te90]:

$$
E_{N}=\left[2 K^{1 / 2}\left(N+\frac{3}{2}\right)\right]^{2 / 3},
$$

and in the non-relativistic limit, $E_{N}-m \ll m$,

$$
E_{N}=\left(N+\frac{3}{2}\right)\left(\frac{2 K}{m}\right)^{1 / 2}+m .
$$

For $A$ non-interacting particles moving in an external harmonic oscillator (3.1), the quantal particle density $\rho(r)$ and total energy $E$ are obtained from Eqs. (3.10)-(3.13) and (3.15) summing over the occupied states. Assuming isospin degeneracy and closed shells,

$$
\begin{aligned}
\rho(r) & =2 \sum_{n, l, j}(2 j+1)\left|\psi_{n l}\right|^{2} \\
& =\frac{1}{\pi r^{2}} \sum_{n, l}\left[(2 l+1) g_{n l}^{2}+(l+1) f_{n l j=l+1 / 2}^{2}+l f_{n l j=l-1 / 2}^{2}\right]
\end{aligned}
$$

and

$$
\begin{aligned}
E+m A & =2 \sum_{n, l, j}(2 j+1) E_{n l}=4 \sum_{n, l}(2 l+1) E_{n l} \\
& =2 \sum_{N}(N+1)(N+2) E_{N} .
\end{aligned}
$$

\subsection{Wigner-Kirkwood Expansion of the Particle Number and the Energy}

In the WK representation, Eqs. (2.60)-(2.62) and (2.66)-(2.68), the semiclassical particle and energy densities become (taking into account that $\nabla m^{*}=\nabla V$ and $\Delta m^{*}=$ $\Delta V):$

$$
\begin{aligned}
\rho_{\mathrm{WK}}(r)=\frac{2 k_{\mathrm{F}}^{3}}{3 \pi^{2}}+\frac{1}{12 \pi^{2}} & {\left[\frac{1}{m^{*} k_{\mathrm{F}}^{3}}\left(m^{*}\left(3 \varepsilon_{\mathrm{F}}^{2}-5 m^{* 2}\right)+\varepsilon_{\mathrm{F}}\left(4 \varepsilon_{\mathrm{F}}^{2}-6 m^{* 2}\right)\right)(\nabla V)^{2}\right.} \\
& \left.-2\left(\frac{1}{k_{\mathrm{F}}}\left(\varepsilon_{\mathrm{F}}+m^{*}\right)+2 \ln \frac{k_{\mathrm{F}}+\varepsilon_{\mathrm{F}}}{m^{*}}\right) \Delta V\right]
\end{aligned}
$$




$$
\begin{aligned}
& \mathcal{E}_{\mathrm{WK}}(r)=\frac{1}{4 \pi^{2}}\left[k_{\mathrm{F}} \varepsilon_{\mathrm{F}}\left(2 \varepsilon_{\mathrm{F}}^{2}-m^{* 2}\right)-m^{* 4} \ln \frac{k_{\mathrm{F}}+\varepsilon_{\mathrm{F}}}{m^{*}}\right] \\
& +\frac{1}{12 \pi^{2}}\left[\left(\frac{1}{k_{\mathrm{F}}^{3}}\left(\varepsilon_{\mathrm{F}}-2 m^{*}\right)\left(\varepsilon_{\mathrm{F}}+m^{*}\right)^{2}-3 \ln \frac{k_{\mathrm{F}}+\varepsilon_{\mathrm{F}}}{m^{*}}\right)(\nabla V)^{2}\right. \\
& \left.-2\left(\frac{1}{k_{\mathrm{F}}}\left(\varepsilon_{\mathrm{F}}+m^{*}\right)\left(2 \varepsilon_{\mathrm{F}}-m^{*}\right)-m^{*} \ln \frac{k_{\mathrm{F}}+\varepsilon_{\mathrm{F}}}{m^{*}}\right) \Delta V\right] \\
& +(V-m) \rho_{\mathrm{WK}}
\end{aligned}
$$

where $k_{\mathrm{F}}$ is related to the chemical potential $\lambda$ by

$$
k_{\mathrm{F}}=(\lambda+m)^{1 / 2}\left(\lambda-m-\Pi r^{2}\right)^{1 / 2},
$$

and $\varepsilon_{F}=\lambda-K r^{2} / 2$.

Eqs. (3.21) and (3.22) can be integrated analytically from $r=0$ to the turning point $r=[(\lambda-m) / K]^{1 / 2}$, to get the semiclassical WK expansion of the particle number $A$ and total energy $E$ up to order $\hbar^{2}$. With the definition $x \equiv(\lambda-m) / 2 m$, we find:

$$
\begin{aligned}
A= & \frac{2}{3} m^{3}\left(\frac{2 m}{K}\right)^{3 / 2}(1+x)^{3 / 2} x^{3}-\frac{m}{2}\left(\frac{2 m}{K}\right)^{1 / 2}(1+x)^{1 / 2} x \\
E= & \frac{128}{3465} m^{4}\left(\frac{2 m}{K}\right)^{3 / 2}(1+x)^{1 / 2}\left[\frac{945}{32} x^{5}+\frac{2905}{64} x^{4}+\frac{1135}{64} x^{3}\right. \\
& \left.+\frac{3}{8} x^{2}-\frac{1}{2} x+1-(1+x)^{-1 / 2}\right] \\
& -\frac{4}{15} m^{2}\left(\frac{2 m}{K}\right)^{1 / 2}(1+x)^{1 / 2}\left[\frac{9}{4} x^{2}+\frac{11}{8} x+1-(1+x)^{-1 / 2}\right] \\
& -m A .
\end{aligned}
$$

Eq. (3.24) is used to determine the chemical potential $\lambda$ which then is inserted into (3.25) to compute the energy. The non-relativistic limit of (3.24) and (3.25) is easily found taking $x \ll 1$. It yields

$$
\begin{aligned}
& A=\frac{1}{12}\left(\frac{2 m}{K}\right)^{3 / 2}(\lambda-m)^{3}-\frac{1}{4}\left(\frac{2 m}{K}\right)^{1 / 2}(\lambda-m) \\
& E=\frac{1}{16}\left(\frac{2 m}{K}\right)^{3 / 2}(\lambda-m)^{4}-\frac{1}{8}\left(\frac{2 m}{K}\right)^{1 / 2}(\lambda-m)^{2}
\end{aligned}
$$




\subsection{Discussion of Results}

As mentioned in the introduction, the Strutinsky averaging method has been successfully employed in non-relativistic nuclear physics to separate the smooth part of the energy from the fluctuating shell corrections [St67, St68, Br73, Je73, Br81, Pr81]. The customary prescription for obtaining the smoothed energy uses single-particle occupation numbers, or the single-particle level density, smoothed by some averaging function. We have also performed Strutinsky averaged (SA) calculations for the relativistic harmonic oscillator of Eq. (3.1). We have used Eq. (3.15) for the single-particle energies and smoothed occupation numbers $\tilde{n}_{i}$ obtained from a Gaussian averaging to get the SA energy

$$
\tilde{E}_{\mathrm{SA}}=\sum_{i} E_{i} \tilde{n}_{i}
$$

The smoothing procedure is the following $\left[\mathrm{Br}_{r} 73, \operatorname{Pr} 81\right]$. We compute the $\tilde{n}_{i}$ as

$$
\tilde{n}_{i}=\int_{-\infty}^{\lambda_{i}} d x f(x)+\sum_{\mu=1}^{M} a_{2 \mu} f^{(2 \mu-1)}\left(\lambda_{i}\right),
$$

where $\lambda_{i}=\left(\lambda-E_{i}\right) / \gamma$, and $\gamma$ is an averaging parameter. For a Gaussian averaging function

$$
f(x)=\frac{1}{\sqrt{\pi}} e^{-x^{2}},
$$

the coefficients $a_{2 \mu}$ are given by

$$
a_{2 \mu}=\frac{(-1)^{\mu}}{2^{2 \mu} \mu !}
$$

and

$$
f^{\mu}(x)=\frac{(-1)^{\mu}}{\sqrt{\pi}} e^{-x^{2}} H_{\mu}(x),
$$

where $H_{\mu}$ are the Hermite polynomials [Ab65]. It has been numerically checked that the so-called plateau condition (stationarity of the shell correction with respect to the smoothing width $\gamma$ ) [Br73],

$$
\left.\frac{\partial \tilde{E}_{\mathrm{SA}}}{\partial \gamma}\right|_{\gamma 0}=0
$$

is guaranteed in each case. This is important, because if (3.33) is not exactly fulfilled, not the entire information about the Strutinsky averaging is contained in the occupation numbers $\tilde{n}_{\mathbf{i}}$ and the quantity $\tilde{E}_{\mathrm{SA}}$ does not give the correct SA energy. 
For the numerical applications we have taken $K=m \omega^{2} / 2$. The reason for this choice is that the non-relativistic limit of this problem is just a set of fermions moving in a potential well given by

$$
V_{\mathrm{NR}}(r)=V(r)+S(r)=\frac{1}{2} m \omega^{2} r^{2} .
$$

Table 3.1 collects the energies obtained from the SA and semiclassical approaches in comparison with the corresponding quantum mechanical (QM) result, for different values of the particle number (closed shells) and of the rest mass of the particles $\left(m=m_{\mathrm{N}}, m=m_{\mathrm{N}} / 100, m=m_{\mathrm{N}} / 1000\right.$, where $\left.m_{\mathrm{N}}=939 \mathrm{MeV}\right)$, with $\hbar \omega=41 A^{-1 / 3}$ $(\mathrm{MeV})$. The semiclassical energies have been calculated from the WK expansion of order $\hbar^{2}, \mathrm{WK} \hbar^{2}$ label, and solving the Euler-Lagrange equations associated with the RETF energy density functional of order $\hbar^{2}, \mathrm{TF} \hbar^{2}$ label (we will discuss this approach later on).

For a non-relativistic harmonic oscillator potential, it has been analytically proven that the SA method and the WK expansion up to order $\hbar^{4}$ are equivalent and yield the same results [Br73, Je73]. Due to the fact that the relativistic eigenvalues (3.15) have a more complicated form than the non-relativistic ones, we have not been able to obtain an analytical expression for the Strutinsky smooth energy. However, the numerical results shown in Table 3.1 seem to indicate that for our model relativistic oscillator the SA and WK methods are also equivalent. The agreement between the SA and WK $\hbar^{2}$ energies is almost perfect irrespective of the value of the rest mass of the particles. The small difference between the SA and WK $\hbar^{2}$ methods could be attributed to the $\hbar^{4}$ contribution that has not been included in the relativistic treatment.

For the $Q M$ results, we see that the relativistic effects on the energy are rather small if the rest mass is the nucleon mass $m_{N}$; the relativistic energy is shifted to smaller values by an amount around $1 \%$. The relativistic corrections become more important when the mass is reduced. With respect to the non-relativistic results, which are independent of $m$, the energy decreases by $\sim 35 \%$ if $m=m_{\mathrm{N}} / 100$ and by $\sim 64 \%$ if $m=m_{N} / 1000$. The SA and semiclassical energies show the same trends as the quantal ones, similar changes are found in their values if the relativistic and non-relativistic results are compared. The difference between the quantal and the SA energy is the socalled shell correction. Table 3.1 shows that it becomes less important when the mass of the particles is reduced. Thus, the shell correction is reduced by the relativistic effects, at least for this example. 
Table 3.1. Total energy for a set of $A$ fermions submitted to the relativistic harmonic oscillator (3.1), with $K=m \omega^{2} / 2, \hbar \omega=41 A^{-1 / 3}(\mathrm{MeV})$ and $m_{\mathrm{N}}=939 \mathrm{MeV}$. The results correspond to quantum mechanical (QM), Strutinsky averaged (SA), $\hbar^{2}$-order Wigner-Kirkwood $\left(W K \hbar^{2}\right)$ and Thomas-Fermi $\left(\mathrm{TF} \hbar^{2}\right)$ calculations, respectively. The rest mass contribution has been subtracted.

\begin{tabular}{crrrrr}
\hline & & \multicolumn{4}{c}{$E(\mathrm{MeV})$} \\
\cline { 3 - 6 } & \multicolumn{1}{c}{$A$} & $\mathrm{QM}$ & \multicolumn{1}{c}{$\mathrm{SA}$} & $\mathrm{WK} \hbar^{2}$ & $\mathrm{TF} \hbar^{2}$ \\
\hline Non-relativistic & 16 & 585.8 & 598.1 & 598.1 & 582.9 \\
& 40 & 1438.6 & 1454.7 & 1454.7 & 1436.1 \\
& 80 & 2854.6 & 2874.5 & 2874.5 & 2852.8 \\
& 140 & 4974.5 & 4998.2 & 4998.2 & 4973.6 \\
& 224 & 7939.2 & 7966.8 & 7966.8 & 7939.4 \\
Relativistic & 16 & 580.0 & 592.0 & 592.0 & 576.8 \\
$m=m_{\mathrm{N}}$ & 40 & 1424.5 & 1439.9 & 1440.2 & 1421.8 \\
& 80 & 2826.7 & 2845.7 & 2846.1 & 2824.7 \\
& 140 & 4925.9 & 4948.8 & 4949.1 & 4925.1 \\
Relativistic & 16 & 385.4 & 390.7 & 391.5 & 378.7 \\
$m=m_{\mathrm{N}} / 100$ & 40 & 948.5 & 955.8 & 956.4 & 941.5 \\
& 80 & 1883.7 & 1893.0 & 1893.4 & 1876.7 \\
& 140 & 3284.1 & 3295.4 & 3295.6 & 3277.2 \\
& 224 & 5242.9 & 5255.9 & 5256.2 & 5236.2 \\
& 16 & 207.1 & 209.7 & 210.1 & 202.3 \\
Relativistic & 4861.7 & 7888.4 & 7888.6 & 7862.2 \\
$m=m_{\mathrm{N}} / 1000$ & 40 & 510.4 & 513.9 & 514.2 & 505.3 \\
& 80 & 1014.3 & 1018.7 & 1018.9 & 1009.1 \\
& 140 & 1768.9 & 1774.2 & 1774.4 & 1763.6 \\
& 224 & 2824.4 & 2830.6 & 2830.8 & 2819.2 \\
\hline
\end{tabular}

In the non-relativistic situation, the scale parameter $r_{0}$ is independent of the energy of the shell but still depends on the mass of the particles, being scaled by a 


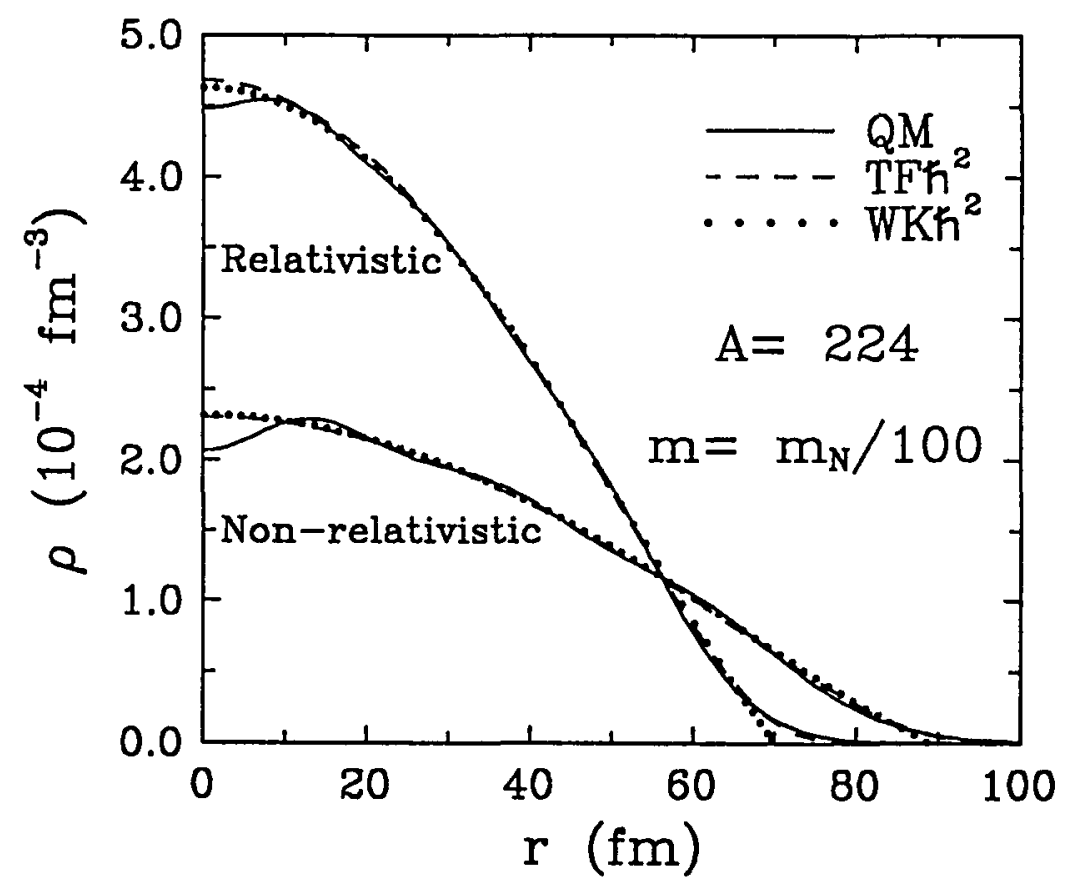

Figure 3.1. Particle densities for the non-relativistic and relativistic harmonic oscillators discussed in the text.

factor $\left(m_{N} / m\right)^{1 / 2}$. In the relativistic case, $r_{0}$ depends on the mass and on the energy of each shell, getting smaller as both quantities increase. This causes the relativistic densities to be pushed to the origin, increasing their central value with respect to the non-relativistic one. This effect is clearly observable in Fig. 3.1, where we plot the relativistic and non-relativistic densities for a harmonic oscillator of $A=224$ particles with $m=m_{\mathrm{N}} / 100$ in the QM, WK $\hbar^{2}$ and $\mathrm{TF} \hbar^{2}$ (see below) approaches. In this case the relativistic $r_{0}$ is reduced by $80 \%$, and the central density increases roughly by a factor 2. The smoothing of the oscillations in the quantal density when the relativistic effects are taken into account can also be noticed. This is in agreement with the trend we have found for the shell correction to the energy.

The semiclassical densities show no oscillations since they lack shell structure. However, they average the quantal densities in the non-relativistic as well as in the relativistic cases. This means that the relativistic effects are properly incorporated by the semiclassical calculation. As it happens in the non-relativistic situation, the WK $\hbar^{2}$ semiclassical densities diverge at the classical turning point and, consequently, have to be regarded more as distributions than as functions. In spite of this, the calculation 
Table 3.2. Same as Table 3.1 keeping the strength of the potential fixed to a constant value $K=K_{N} \equiv m_{N}\left(41 A^{-1 / 3}\right)^{2} / 2$.

\begin{tabular}{crrrrr}
\hline & & \multicolumn{4}{c}{$E(\mathrm{MeV})$} \\
\cline { 3 - 6 } & \multicolumn{1}{c}{$A$} & \multicolumn{1}{c}{$\mathrm{QM}$} & \multicolumn{1}{c}{$\mathrm{SA}$} & \multicolumn{1}{c}{$\mathrm{WK}^{2}$} & $\mathrm{TF}^{2}$ \\
\hline Relativistic & 16 & 2070.7 & 2096.9 & 2100.5 & 2022.9 \\
$m=m_{\mathrm{N}} / 100$ & 40 & 5103.8 & 5139.2 & 5141.9 & 5053.2 \\
& 80 & 10142.5 & 10186.9 & 10189.2 & 10090.5 \\
& 140 & 17688.7 & 17742.0 & 17743.9 & 17636.1 \\
& 224 & 28243.8 & 28306.0 & 28307.7 & 28191.6 \\
Relativistic & 16 & 2156.3 & 2182.2 & 2186.3 & 2101.9 \\
$m=m_{\mathrm{N}} / 1000$ & 40 & 5317.5 & 5353.0 & 5355.9 & 5259.8 \\
& 80 & 10569.7 & 10614.2 & 10616.6 & 10510.3 \\
& 140 & 18436.0 & 18489.4 & 18491.6 & 18375.9 \\
& 224 & 29439.3 & 29501.6 & 29503.6 & 29379.3 \\
\hline
\end{tabular}

of expectation values of operators is well defined as it happens, for instance, with the particle number (3.24) and the energy (3.25). Note the steep fall-off of the WK $\hbar^{2}$ densities in Fig. 3.1; we have put a cutoff at the turning point where they become negative.

The results reported in Table 3.1 and Fig. 3.1 have been obtained with a potential well whose strength $K$ depends on the rest mass $m$ of the particles. We have also made calculations changing $m$ but keeping the strength fixed to a constant value $K=K_{\mathrm{N}} \equiv m_{\mathrm{N}}\left(41 A^{-1 / 3}\right)^{2} / 2$. The corresponding results are displayed in Table 3.2. In this case one finds the same trends as discussed above comparing the semiclassical with the quantal results. It is remarkable the excellent agreement between $W K \hbar^{2}$ and SA.

Finally, we would like to pay some attention to the $T F \hbar^{2}$ results obtained applying the variational principle to the energy density functional (2.80). The corresponding Euler-Lagrange equation

$$
\frac{\delta \mathcal{E}}{\delta \rho}=\frac{\partial \mathcal{E}}{\partial \rho}-\nabla \frac{\partial \mathcal{E}}{\partial(\nabla \rho)}=\lambda
$$




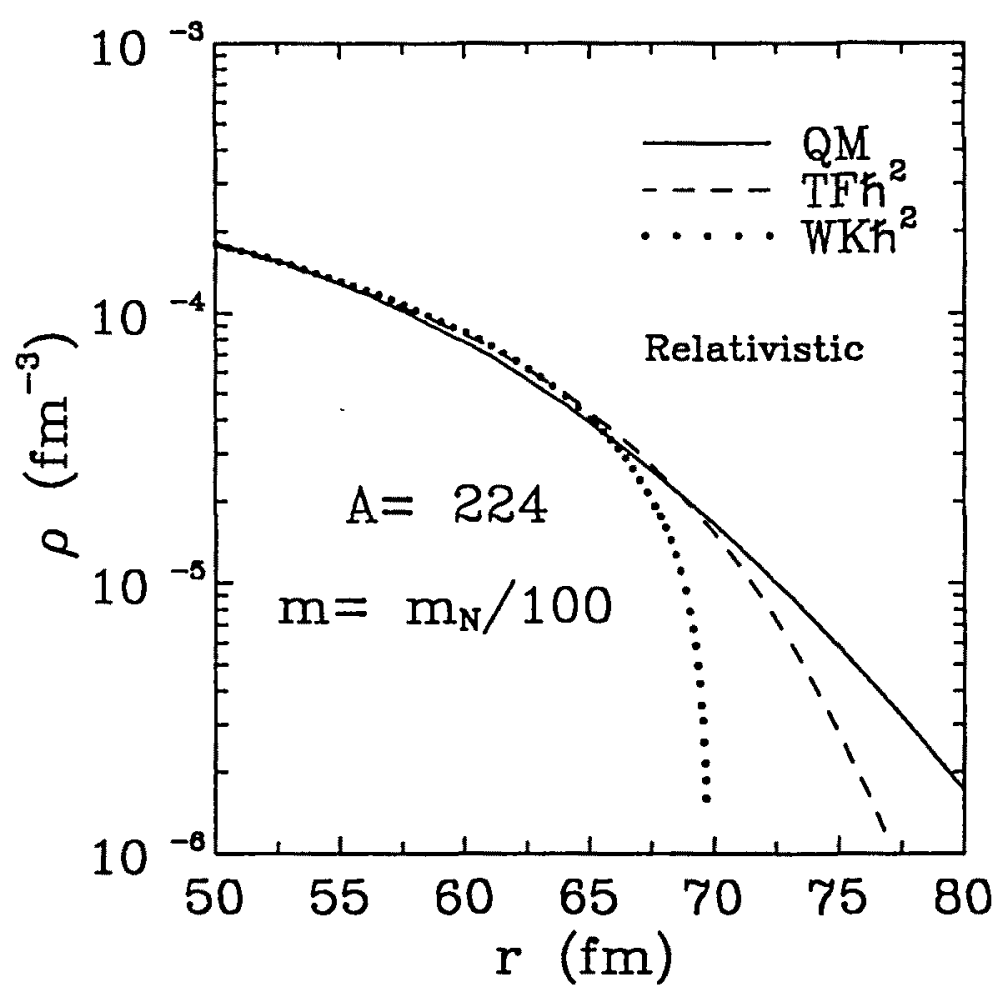

Figure 3.2. Semi-logarithmic plot of the density tails corresponding to the relativistic harmonic oscillator of Fig. 3.1.

is solved ${ }^{\dagger}$ using the imaginary time-step method (insight into its practical implementation can be gained from Subsection 4.2.3 in the following chapter).

As it happens in the non-relativistic case, the TF $\hbar^{2}$ method is not equivalent to the $\mathrm{WK} \hbar^{2}$ one (see the difference between the $W K \hbar^{2}$ and $\mathrm{TF} \hbar^{2}$ results in Table 3.1), because they originate from different rearrangements in the $\hbar$ expansion of the energy functional. Consequently, since we have numerically shown that the WK and SA methods are equivalent for the harmonic oscillator we are considering, the smooth part of the energy is not so properly estimated by the TF $\hbar^{2}$ method. Let us mention, however, that the divergence problems in the classically forbidden region are not present in the TF $\hbar^{2}$ functionals. This is an advantage of $\mathrm{TF} \hbar^{2}$ over the $\mathrm{WK} \hbar^{2}$ method. The divergent behaviour of $\mathrm{WK}^{2}$ at the turning point can be seen in Fig. 3.2, where we have plotted the density tails corresponding to Fig. 3.1 on a semi-logarithmic scale.

${ }^{\dagger}$ The author wishes to express again his acknowledgement to Martí $\mathrm{Pi}$, whose expertise made possible the development of the computer codes needed to solve the semiclassical variational equations. 
From Tables 3.1 and $3.2 \mathrm{it}$ is seen that the $\mathrm{WK} \hbar^{2}$ and SA approximations underbind the system with respect to the quantal result regardless of the mass of the particles. On the contrary, the TF $\hbar^{2}$ approximation generally yields some overbinding. 


\section{Chapter 4}

\section{Semiclassical Description of Nuclear Systems in Relativistic Mean Field Models}

IN CHAPTER 3 we have applied the semiclassical scheme to the discussion of a relativistic harmonic oscillator. From the study of this model external potential, we can conclude that the semiclassical expansion bears all the relativistic ingredients for positive energy states, the difference with quantal results being due to shell effects. This satisfactory background suggests using the method on a broader scale and in a more realistic context. In Chapters 4 and 5 we shall address the calculation of properties of nuclear systems with the $\hbar^{2}$-order RETF functionals.

This chapter is concerned with relativistic non-linear $\sigma-\omega$ models in mean field approximation. After a summary of some general aspects of the $\sigma-\omega$ model, we consider the calculation of finite nuclei. First, we introduce the Lagrangians and derive the corresponding mean field energy densities and variational equations in both the semiclassical and the quantal Hartree approach, paying special attention to the former. Then we sketch out the numerical method we have employed to tackle the semiclassical variational equations of order $\hbar^{2}$, and discuss at length the results for finite nuclei that we have obtained with different parametrizations of the $\sigma-\omega$ model. Finally, in the 
last section of the chapter we give an account of the surface properties (density profiles, and surface and curvature energies) obtained in semi-infinite nuclear matter from the RETF model.

\subsection{Overview}

A short sketch of the $\sigma-\omega$ model has been given in the introduction. We now mention that in the original mean field linear $\sigma-\omega$ model of Walecka the incompressibility of nuclear matter, $\kappa=545 \mathrm{MeV}$, is considerably too large as compared to any empirical values [B180, Sh88], giving rise to an equation of state too stiff. The inclusion by Boguta and Bodmer [Bo77b] of non-linear contributions through cubic and quartic terms in the scalar field, shifted the incompressibility to more reasonable values in comparison with the empirical data. Moreover, the Coulomb repulsion and an isovector $\rho$ meson have to be included in the Lagrangian to account for proton-neutron asymmetry effects in any realistic description of nuclei. The non-linear model has been widely used in the recent applications (see e.g. [Re86, Ga90a] and [Bo83a, Bo83b, Gl83, Bo84b, We89, Hi91]), inasmuch as it yields a successful description of the surface and ground-state properties of finite nuclei (e.g., binding energies, spin-orbit splittings, density distributions, r.m.s. radii or isotope shifts).

In the model with cubic and quartic scalar self-interactions, the effective mass at saturation is rather small $\left(m^{*} / m \sim 0.60\right)$ if compared to the value extracted from optical model analyses $\left(m^{*} / m \approx 0.83\right)$ [Jo87] or to the value of a realistic Skyrme force like $\mathrm{SkM}^{*}\left(m^{*} / m=0.79\right)$ [Ba82, Br85]. Recently, Zimanyi and Moszkowski [Zi90] have proposed an alternative type of non-linearity, generated by a derivative coupling between the scalar and the fermion fields. In contrast to the model of Boguta and Bodmer, this non-linearity does not introduce extra free parameters. The derivative scalar coupling yields an acceptable incompressibility and a larger effective mass, $m^{*} / m=0.85$, but it gives too small a value for the spin-orbit splitting. In fact, the spin-orbit splitting in light nuclei determines $m^{*} / m$ within quite narrow limits to be $\sim 0.60$, almost independently of the form of the scalar coupling [Bo89]. More general types of non-linear couplings of the scalar $\sigma$ meson have been investigated [Re88, Fe91, Ko91].

The relativistic mean field model has not only been used to describe ground-state properties of spherical nuclei but also to study axially symmetric deformed nuclear shapes [Le86, Pa87, Pr87, Ga90a, Zh91a], giant resonances [Ho81a, Pr85, Hu90, Bo91], 
magnetic moments [Bo84a, Ho88a], scattering processes of nucleons on nuclei at intermediate energies [Cl83, Ne83], or in dynamical calculations of nuclear collisions [Mü81, Cu85]. Calculations with mean field Lagrangians whose parameters are fitted to DiracBrueckner self-energies obtained in nuclear matter have also been performed [Ma89, El90, Gm91, Br92]. The role of vacuum polarization effects in nuclear matter and in finite spherical and deformed nuclei has been examined in several works (see e.g. [Ch77; Se78, Ho84, Ho87, Gl89, Zh91b]).

One has to admit, however, that the number of experimental nuclear properties investigated within the framework of the relativistic mean field theory is so far considerably smaller than those investigated and successfully reproduced by conventional density-dependent $\mathrm{HF}$ calculations.

The quality of the relativistic mean field results turns out to be very similar to that of the corresponding results of conventional HF calculations using Skyrme forces. In any of both theories, the experimental spectrum is generally more compressed as compared to that obtained in the calculations. The oscillations in the interior of the calculated charge density distributions are only somewhat milder with respect to those usually obtained in non-relativistic DDHF. Nevertheless, the relativistic mean field results are in slight better agreement with experiment and, therefore, are found to have an edge over their non-relativistic counterparts.

One may attribute the relative success of the simple relativistic mean field model to the intrinsic Lorentz covariance of the theory, which leads naturally to densitydependent interactions between nucleons. Furthermore, non-relativistic reduction reveals non-central and non-local features inherent in the mean field formalism. An obvious advantadge of a relativistic approach is the treatment of the spin-orbit force, which emerges just out from the interplay between the upper and the lower components. In relativistic mean field theory, the spin-orbit interaction is given in terms of the scalar and the vector fields, whose strength is adjusted from the nuclear matter calculations. This leaves no free fitting of the spin-orbit force. The situation should be contrasted with Skyrme interactions where the density dependence of the interaction is phenomenological at the outset, and the spin-orbit force has to be build in by hand and its strength adjusted. A discussion of these aspects and of some merits and shortcomings of the relativistic mean field model may be found, e.g., in [Ho81b, Re86, Se86, Ri88, Re89, Ga90a]. 


\subsection{Calculations of Finite Nuclei}

The question remains of the comparison between semiclassical and quantal results in the relativistic framework when the fields and the densities are self-consistently generated. It is the purpose of this section to investigate and discuss this point. In the non-relativistic case, such comparisons have underlined the reliability of semiclassical methods [Br85, Ce90a]. We shall present here binding energies, sizes and density distributions of finite nuclei calculated in the semiclassical approximations of order $\hbar^{0}$ and $\hbar^{2}$, as well as the corresponding quantal Hartree solutions.

In order to establish useful comparisons, we have considered two extensions of the Walecka Lagrangian reproducing sufficiently well the bulk nuclear matter data. The first one is the non-linear $\sigma-\omega$ model proposed by Boguta and Bodmer. Among several possibilities, we have chosen two recent sets of parameters [Sh91b], denoted SRK3M5 and SRK3M7, for their ability to fit accepted nuclear matter data. They differ through their effective mass and surface properties. To complement the study, we also perform calculations with other two sets of parameters. Following [Ra90], we call them HII [Bo82] and HIV [Gl87]. As an alternative, the derivative coupling model of Zimany and Moszkowski [Zi90] (see also [Ba91, Fe91, Ko91, Sh91a]) has been considered. For both models, the results of the semiclassical approach will be compared with those obtained in the Hartree approximation.

\subsubsection{Lagrangians and energy densities}

The notation closely follows that of Serot and Walecka [Se86], and will not be explicited here. The extended Walecka Lagrangian advocated by Boguta and Bodmer (BB) [Bo77b], which will be treated in mean field approximation, can be written as

$$
\mathcal{L}_{\mathrm{BB}}=\mathcal{L}-\frac{1}{3} b \phi^{3}-\frac{1}{4} c \phi^{4}
$$

where

$$
\begin{aligned}
\mathcal{L}= & \bar{\psi}\left[\gamma_{\mu}\left(i \partial^{\mu}-g_{v} V^{\mu}\right)-m^{*}\right] \psi+\frac{1}{2}\left(\partial_{\mu} \phi \partial^{\mu} \phi-m_{\mathrm{s}}{ }^{2} \phi^{2}\right) \\
& -\frac{1}{4} F_{\mu \nu} F^{\mu \nu}+\frac{1}{2} m_{\mathrm{v}}{ }^{2} V_{\mu} V^{\mu}
\end{aligned}
$$

with

$$
F_{\mu \nu}=\partial_{\mu} V_{\nu}-\partial_{\nu} V_{\mu}
$$


The effective mass $m^{*}$ is related to the scalar field $\phi$ by

$$
m^{*}=m-g_{\mathbf{s}} \phi
$$

In accordance with the one boson exchange (OBE) description of the NN interaction, this Lagrangian incorporates the nucleonic degrees of freedom through the baryonic field $\psi$ and the mesonic contributions by means of an isoscalar-vector $\omega$ boson (the field $V_{\mu}$ ) and an isoscalar-scalar $\sigma$ boson (the field $\phi$ ). The existence of such a $\sigma$ boson, with a mass in the area of $400-700 \mathrm{MeV}$, is not supported by any experimental evidence. However, this (fictious) neutral scalar meson is crucial to provide the intermediate-range attraction of the nuclear force in OBE potentials, and it is interpreted as simulating in part two-pion exchange contributions to the NN force [Ma89]. A $\pi$-meson field is absent in the Lagrangian (4.2) because it does not contribute in a mean field description of bulk properties of the ground state of nuclei [Ho81b, Re86, Se86].

The Zimanyi-Moszkowski (ZM) Lagrangian [Zi90] is simply $\mathcal{L}_{\mathrm{ZM}}=\mathcal{L}$, but in this case the non-linearity is contained in the connection between the effective mass and the scalar field, which reads

$$
m^{*}=\frac{m}{1+g_{s} \phi / m} .
$$

Thus it requires no extra terms, and consequently this Lagrangian is dealing with less free parameters. At low density, the two kinds of non-linearity are of the same nature, and they would be equivalent up to first order in $\phi$ [Zi90]. They are clearly different as $\phi$ gets large.

The two above Lagrangians are merely dealing with symmetric matter. In order to describe actual nuclei, apart from the neutral $\sigma$ and $\omega$ mesons, it is necessary to introduce proton-neutron asymmetry effects. This is done by adding the $\rho$-meson contribution and the electromagnetic field, namely

$$
\begin{aligned}
\mathcal{L}^{\prime}= & \mathcal{L}-\frac{1}{4} \mathbf{G}_{\mu \nu} \cdot \mathrm{G}^{\mu \nu}+\frac{1}{2} m_{\rho}{ }^{2} \mathbf{b}_{\mu} \cdot \mathbf{b}^{\mu}-\frac{1}{2} g_{\rho} \bar{\psi} \gamma_{\mu} \tau \cdot \mathbf{b}^{\mu} \psi \\
& -\frac{1}{4} H_{\mu \nu} H^{\mu \nu}-e \bar{\psi} \gamma_{\mu} \frac{1}{2}\left(1+\tau_{3}\right) A^{\mu} \psi,
\end{aligned}
$$

where

$$
\mathbf{G}_{\mu \nu}=\partial_{\mu} \mathbf{b}_{\nu}-\partial_{\nu} \mathbf{b}_{\mu}, \quad H_{\mu \nu}=\partial_{\mu} A_{\nu}-\partial_{\nu} A_{\mu}
$$


Note that $b_{\mu}$ is a four vector in Minkowski space and a three vector in isospin space. As mentioned, this is a phenomenological effective model. The $\sigma$-meson mass $m_{\mathbf{s}}$, the coupling constants $g_{s}, g_{\mathrm{v}}$ and $g_{\rho}, b$ and $c$ are the free parameters of the Lagrangian. They are usually adjusted to reproduce the properties of nuclear matter and finite nuclei. From this standpoint, some features and contributions not included in the Lagrangian (4.6), or suppressed later by the mean field approximation, are indirectly built into the normalized values of the parameters of the model. These effective parameters turn out to be essentially density-independent, at least over a moderate range of densities.

The energy density is derived in a standard fashion from the Lagrangian. The quantum structure is expressed as usual by expanding the nucleon field on a single particle basis $\varphi_{\alpha}$. In the static Hartree approximation it yields

$$
\mathcal{E}_{\mathrm{BB}}^{\mathrm{H}}=\mathcal{E}^{\mathrm{H}}+\frac{1}{3} b \phi_{\circ}^{3}+\frac{1}{4} c \phi_{\circ}^{4}
$$

for the BB Lagrangian. The first term in the r.h.s. of (4.8) is given by

$$
\begin{aligned}
\mathcal{E}^{\mathrm{H}}= & \sum_{\alpha} \varphi_{\alpha}^{\dagger}\left[-i \alpha \cdot \nabla+\beta m^{*}-m+g_{\mathrm{v}} V_{0}+\frac{1}{2} g_{\rho} \tau_{3} b_{0}+e \frac{1}{2}\left(1+\tau_{3}\right) A_{0}\right] \varphi_{\alpha} \\
& +\mathcal{E}_{\mathrm{f}}
\end{aligned}
$$

with $m^{*}=m-g_{s} \phi_{\circ}$ and

$$
\begin{aligned}
\mathcal{E}_{\mathrm{f}}= & \frac{1}{2}\left[\left(\nabla \phi_{\mathrm{o}}\right)^{2}+m_{\mathrm{s}}{ }^{2} \phi_{\mathrm{o}}^{2}\right]-\frac{1}{2}\left[\left(\nabla V_{0}\right)^{2}+{m_{\mathrm{v}}}^{2} V_{0}^{2}\right] \\
& -\frac{1}{2}\left[\left(\nabla b_{0}\right)^{2}+m_{\rho}{ }^{2} b_{0}^{2}\right]-\frac{1}{2}\left(\nabla A_{0}\right)^{2} .
\end{aligned}
$$

Similarly, we have

$$
\varepsilon_{\mathrm{ZM}}^{\mathrm{H}}=\mathcal{E}^{\mathrm{H}}
$$

for the ZM model, now with $m^{*}=m\left(1+g_{\mathrm{s}} \phi_{\mathrm{o}} / m\right)^{-1}$.

In the foregoing Hartree energy densities the meson fields have been approximated by their ground-state expectation values. As in the majority of current applications, we restrict the index $\alpha$ to run only over occupied shell model orbitals of the positive spectrum and neglect any contribution from antiparticles (no sea approximation). No spatial components of the vector meson and photon fields appear due to time reversal symmetry, as in this case there are no currents in the nucleus and only the time-like components survive. Charge conservation guarantees that only the third component 
(in isospin space) of the isovector current $b_{0}$ does not vanish. As pointed out in [Ho88a, Ga90a, Sp92], consideration of the full four-component structure of the vector meson fields may play an important role in all cases where currents are involved, for instance, in describing magnetic moments as well as in rotating nuclei.

The corresponding semiclassical energy densities have a very similar structure, except that the nucleon variables are now the proton and neutron densities. One evaluates the TF $\hbar^{2}$ energy density corresponding to (4.9) from Eq. (2.80):

$$
\mathcal{E}^{\mathrm{sc}}=\mathcal{E}_{0}+\mathcal{E}_{2}+g_{\mathrm{v}} V_{0} \rho+\frac{1}{2} g_{\rho} b_{0}\left(\rho_{p}-\rho_{n}\right)+e A_{0} \rho_{p}+\mathcal{E}_{\mathfrak{f}}
$$

with

$$
\mathcal{E}_{0}=\sum_{q} \frac{1}{8 \pi^{2}}\left[k_{\mathrm{F}} \varepsilon_{\mathrm{F}}^{3}+k_{\mathrm{F}}^{3} \varepsilon_{\mathrm{F}}-m^{* 4} \ln \frac{k_{\mathrm{F}}+\varepsilon_{\mathrm{F}}}{m^{*}}\right]_{q}-m \rho
$$

and

$$
\begin{aligned}
\mathcal{E}_{2}=\sum_{q}[ & B_{1 q}\left(k_{F_{q}}, m^{*}\right)\left(\nabla \rho_{q}\right)^{2}+B_{2 q}\left(k_{F q}, m^{*}\right)\left(\nabla \rho_{q} \cdot \nabla m^{*}\right) \\
& \left.+B_{3 q}\left(k_{F_{q}}, m^{*}\right)\left(\nabla m^{*}\right)^{2}\right]
\end{aligned}
$$

The functions $B_{i q}$ are, cf. Eq. $(2.80)$,

$$
\begin{aligned}
& B_{1 q}\left(k_{\mathrm{F} q}, m^{*}\right)=\left[\frac{\pi^{2}}{24 k_{\mathrm{F}}^{3} \varepsilon_{\mathrm{F}}^{2}}\left(\varepsilon_{\mathrm{F}}+2 k_{\mathrm{F}} \ln \frac{k_{\mathrm{F}}+\varepsilon_{\mathrm{F}}}{m^{*}}\right)\right]_{q} \\
& B_{2 q}\left(k_{\mathrm{F} q}, m^{*}\right)=\left[\frac{m^{*}}{6 k_{\mathrm{F}} \varepsilon_{\mathrm{F}}^{2}} \ln \frac{k_{\mathrm{F}}+\varepsilon_{\mathrm{F}}}{m^{*}}\right]_{q} \\
& B_{3 q}\left(k_{\mathrm{F} q}, m^{*}\right)=\left[\frac{k_{\mathrm{F}}^{2}}{24 \pi^{2} \varepsilon_{\mathrm{F}}^{2}}\left(\frac{\varepsilon_{\mathrm{F}}}{k_{\mathrm{F}}}-\left(2+\frac{\varepsilon_{\mathrm{F}}^{2}}{k_{\mathrm{F}}^{2}}\right) \ln \frac{k_{\mathrm{F}}+\varepsilon_{\mathrm{F}}}{m^{*}}\right)\right]_{q} .
\end{aligned}
$$

The subscript $q$ denotes the charge state of each nucleon, $\rho=\rho_{p}+\rho_{n}$ is the particle density, $k_{\mathrm{F}_{q}}=\left(3 \pi^{2} \rho_{q}\right)^{1 / 3}$ is the Fermi momentum and $\varepsilon_{\mathrm{F}_{q}}=\left(k_{\mathrm{F}_{q}}{ }^{2}+m^{* 2}\right)^{1 / 2}$.

The semiclassical energy density for the two considered models is given by

$$
\mathcal{E}_{\mathrm{BB}}^{\mathrm{sc}}=\mathcal{E}^{\mathrm{sc}}+\frac{1}{3} b \phi_{0}^{3}+\frac{1}{4} c \phi_{\mathrm{o}}^{4}
$$

and

$$
\mathcal{E}_{\mathrm{ZM}}^{\mathrm{sc}}=\mathcal{E}^{\mathrm{sc}}
$$




\subsubsection{Variational equations}

In the semiclassical approach, the ground-state densities and the meson and photon fields are obtained by solving the Euler-Lagrange equations ensuing from the variational principle applied to (4.18) or (4.19):

$$
\begin{gathered}
\varepsilon_{\mathrm{Fq}}-m+g_{\mathrm{v}} V_{0}+e A_{0}+\frac{1}{2} g_{\rho} b_{0}-2 B_{1 q} \Delta \rho_{q}-B_{2 q} \Delta m^{*}-\frac{\partial B_{1 q}}{\partial \rho_{q}}\left(\nabla \rho_{q}\right)^{2} \\
-2 \frac{\partial B_{1 q}}{\partial m^{*}}\left(\nabla \rho_{q} \cdot \nabla m^{*}\right)-\left(\frac{\partial B_{2 q}}{\partial m^{*}}-\frac{\partial B_{3 q}}{\partial \rho_{q}}\right)\left(\nabla m^{*}\right)^{2}-\lambda_{q}=0 \\
\Delta A_{0}=-e \rho_{\mathrm{p}} \\
\left(\Delta-m_{\rho}^{2}\right) b_{0}=-\frac{1}{2} g_{\rho}\left(\rho_{\mathrm{p}}-\rho_{\mathrm{n}}\right) \\
\left(\Delta-m_{\mathrm{v}}^{2}\right) V_{0}=-g_{\mathrm{v}} \rho \\
\left(\Delta-m_{\mathrm{s}}^{2}\right) \phi_{0}=-g_{\mathrm{s}} \rho_{\mathrm{s}} \xi+\delta
\end{gathered}
$$

where the semiclassical scalar density is

$$
\begin{gathered}
\rho_{\mathbf{s}}=\frac{\partial \varepsilon_{0}}{\partial m^{*}}-\sum_{q}\left[B_{2 q} \Delta \rho_{q}+2 B_{3 q} \Delta m^{*}+\left(\frac{\partial B_{2 q}}{\partial \rho_{q}}-\frac{\partial B_{1 q}}{\partial m^{*}}\right)\left(\nabla \rho_{q}\right)^{2}\right. \\
\left.+2 \frac{\partial B_{3 q}}{\partial \rho_{q}}\left(\nabla \rho_{q} \cdot \nabla m^{*}\right)+\frac{\partial B_{3 q}}{\partial m^{*}}\left(\nabla m^{*}\right)^{2}\right] .
\end{gathered}
$$

The quantities $\xi$ and $\delta$ are defined as $\xi=1, \delta=b \phi_{0}^{2}+c \phi_{0}^{3}$ for the BB Lagrangian, and $\xi=\left(m^{*} / m\right)^{2}, \delta=0$ for the ZM Lagrangian. The explicit evaluation of $\rho_{\mathbf{s}}$ from (4.25) leads to the expression already given in (2.79). As they have been obtained in two distinct ways, this gives further confidence on the correctness of the derived semiclassical equations.

The classical variational principle applied to the Lagrangian (4.6), in mean field static Hartree approximation, gives the equation of motion for the quantal fields, i.e., the Dirac equation for the nucleons

$$
\left[-i \alpha \cdot \nabla+\beta m^{*}-m+g_{v} V_{0}+\frac{1}{2} g_{\rho} \tau_{3} b_{0}+e \frac{1}{2}\left(1+\tau_{3}\right) A_{0}\right] \varphi_{\alpha}=\varepsilon_{\alpha} \varphi_{\alpha}
$$


and the Klein-Gordon equations for the mesons, which are the same as the set (4.21)-(4.24) with the source terms

$$
\begin{aligned}
\rho_{q} & =\sum_{\alpha} \varphi_{\alpha}^{\dagger} \frac{1}{2}\left(1 \pm \tau_{3}\right) \varphi_{\alpha} \\
\rho_{s} & =\sum_{\alpha} \varphi_{\alpha}^{\dagger} \gamma^{0} \varphi_{\alpha} .
\end{aligned}
$$

Let us now come back to a point which was put forward at the end of Subsection 2.4.2, when we were concerned with the choice of the inversion scheme to obtain the relativisic density functionals from the semiclassical Wigner-Kirkwood expansions. In contradistinction to the case of external potentials, the scalar field $\phi_{0}$ (and the effective mass $m^{*}$ ) is here self-consistently determined, receiving an explicit contribution of order $\hbar^{2}$ through $\rho_{s}$ in Eq. (4.24). Therefore, one might think that $m^{*}$ appearing in $\mathcal{E}_{0}$ should be replaced by its expansion in a pure zero order term $m_{0}^{*}$ and a correction of order $\hbar^{2}, \delta m^{*}=m^{*}-m_{0}^{*}+\mathcal{O}\left(\hbar^{4}\right)$, as discussed in [Sp92]. If one wants to follow this route, one should proceed in a similar way as the one used in Subsection 2.4 .2 to obtain the correction $\delta k$ to the local Fermi momentum. Expanding the scalar density around $k_{0}$ and $m_{0}^{*}$

$$
\begin{aligned}
\rho_{\mathbf{s}}\left(k_{\mathrm{F}}, m^{*}\right)= & \rho_{\mathbf{s}, 0}\left(k_{0}, m_{0}^{*}\right)+\left(\frac{\partial \rho_{\mathbf{s}, 0}\left(k_{0}, m_{0}^{*}\right)}{\partial k_{0}}\right) \delta k \\
& +\left(\frac{\partial \rho_{\mathbf{s}, 0}\left(k_{0}, m_{0}^{*}\right)}{\partial m_{0}^{*}}\right) \delta m^{*}+\rho_{\mathbf{s}, 2}\left(k_{0}, m_{0}^{*}\right)+\mathcal{O}\left(\hbar^{4}\right)
\end{aligned}
$$

and imposing

$$
\rho_{\mathrm{s}, 0}\left(k_{0}, m_{0}^{*}\right)=\rho_{\mathrm{s}}\left(k_{\mathrm{F}}, m^{*}\right)
$$

Eq. (4.28) leads to

$$
\delta m^{*}=-\left[\rho_{\mathrm{s}, 2}\left(k_{0}, m_{0}^{*}\right)-\frac{m_{0}^{*}}{\varepsilon_{0}} \rho_{2}\left(k_{0}, m_{0}^{*}\right)\right]\left(\frac{\partial \rho_{\mathrm{s}, 0}\left(k_{0}, m_{0}^{*}\right)}{\partial m_{0}^{*}}\right)^{-1}
$$

finally replacing $k_{0}$ by $k_{\mathrm{F}}$ (we drop the subscript $q$ to ease the notation). Then, the semiclassical energy density (4.12) would have an extra contribution:

$$
\mathcal{E}^{\mathrm{sc}}\left(k_{\mathrm{F}}, m^{*}\right)=\mathcal{E}^{\mathrm{sc}}\left(k_{\mathrm{F}}, m_{0}^{*}\right)+\left(\frac{\partial \mathcal{E}_{0}\left(k_{\mathrm{F}}, m_{0}^{*}\right)}{\partial m_{0}^{*}}\right) \delta m^{*}
$$


The variational equations obtained from this new functional are formally the same as the set (4.20)-(4.24) replacing $m^{*}$ by $m_{0}^{*}$ and $\rho_{s}$ by $\rho_{s, 0}\left(k_{F}, m_{0}^{*}\right)$, except for Eq. (4.20) which in his 1.h.s. has a new term

$$
\left(\frac{\partial \rho_{s}\left(k_{\mathrm{F}}, m_{0}^{*}\right)}{\partial k_{\mathrm{F}}}\right) \frac{\pi^{2}}{k_{\mathrm{F}}^{2}} \delta m^{*}
$$

Proceeding in this manner, we have the following additional relation which allows us to obtain $m_{0}^{*}$ from $\phi_{0}$ :

$$
m_{0}^{*}+\delta m^{*}=m^{*}= \begin{cases}m-g_{\mathrm{s}} \phi_{\mathrm{o}} & (\mathrm{BB}) . \\ m\left(1+g_{\mathrm{s}} \phi_{\mathrm{o}} / m\right)^{-1} & (\mathrm{ZM}) .\end{cases}
$$

However, it is easy to realize that this decomposition of $m^{*}$ is not compulsory to get the self-consistent densities, meson fields and energies up to order $\hbar^{2}$ : the same EulerLagrange equations are obtained expanding around $m_{0}^{*}$ in the former set (4.20)-(4.24) to order $\hbar^{2}$. Thus, the solutions of the original set of variational equations (4.20)-(4.24) implicitly contain the correction $\delta m^{*}$ and, for all practical purposes, are the same as those which would be obtained starting from the functional (4.31). In addition, the technical problem of solving the variational equations simplifies if one does not have to go through Eq. (4.33) to obtain $m_{0}^{*}$ at each iteration step.

\subsubsection{Numerical solution}

This subsection is devoted to questions concerning the numerical treatment of the semiclassical variational equations of second order ${ }^{\dagger}$. The field equations (4.21)-(4.24) together with the nucleon equation (4.20) constitute a coupled set of equations which ought to be solved simultaneously. The non-linear gradient terms in $\rho$ and $m^{*}$ make the numerical approach rather delicate. These difficulties notwithstanding, the task much resembles non-relativistic ETF calculations with Skyrme forces and similar iterative schemes can be employed.

To solve the nucleon equation (4.20) we rely on the so-called imaginary time-step method (ITS) [Da80, Le84, Da85a]. It is inspired from the way time-dependent HF equations are solved [Bo76b, Da81], and it is currently used in static non-relativistic

\footnotetext{
'We shall not present here further details about the numerical handling of the relativistic quantal Hartree equations, as this has been amply discussed in the literature (see e.g. [Ho81b, Se86, Re86, Re89, Ga90]).
} 
HF and ETF calculations. The time-dependent equation

$$
i \frac{\partial}{\partial t} \varphi=h \varphi
$$

is formally solved as

$$
\varphi(\Delta t)=\exp (-i \Delta t h) \varphi(0)
$$

If the time step $\Delta t$ is replaced by an imaginary quantity $-i \Delta \tau$, the repeated action of the exponential

$$
\varphi^{(n+1)}=\exp (-\Delta \tau h) \varphi^{(n)}
$$

followed by a normalization of $\varphi$, causes the wave function to converge to the lowest eigenstate of $h$ [Da80].

In the semiclassical problem we recast Eq. $(4.20), \delta \mathcal{E}^{\mathrm{sc}} / \delta \rho_{q}=\lambda_{q}$, into the form

$$
\left(-\frac{\xi}{2 m} \Delta+W_{q}\right) \Phi_{q}=\lambda_{q} \Phi_{q}
$$

by introducing $\Phi_{q}=\rho_{q}^{1 / 2}$ and

$$
W_{q}=\frac{\delta \mathcal{E}^{\mathrm{sc}}}{\delta \rho_{q}}+\frac{\xi}{2 m} \frac{\Delta \Phi_{q}}{\Phi_{q}}
$$

where $\xi$ is an arbitrary constant. This is an eigenvalue equation of Schrödinger type for the ETF single-particle hamiltonian $h_{\mathrm{ETF}}=-\xi \Delta / 2 m+W_{q}$ which may be solved by application of the ITS method [Le84, Da85a].

The differential operators, densities and fields are discretized on a finite mesh in coordinate space. In practice one works with a $[1,1]$ Padé approximation to the exponential. The algorithm then reads

$$
\left(1+\frac{\Delta \tau}{2} h_{\mathrm{ETF}}^{(n+1 / 2)}\right) \Phi_{q}^{(n+1)}=\left(1-\frac{\Delta \tau}{2} h_{\mathrm{ETF}}^{(n+1 / 2)}\right) \Phi_{q}^{(n)},
$$

where $h_{\mathrm{ETF}}^{(n+1 / 2)}$ is calculated in an intermediate step between $n$ and $n+1$ (see [Bo76b, Da80, Da81]). The Coulomb potential $A_{0}$ and the Yukawa potentials $b_{0}, V_{0}$ and $\phi_{0}$ required at each iteration are evaluated by solving, respectively, the discrete Poisson and Helmholtz equations using Gaussian elimination [Bo76b, Da81]. 
The whole procedure can thus be described as follows:

(i) Start from Fermi-shaped densities $\rho_{q}$ and $\rho_{\mathbf{s}}$.

(ii) Arrived at the $n$ th-iteration stage, determine the scalar meson potential $\phi_{0}^{(n+1)}$ by Gaussian elimination of the discretized radial equation, cf. (4.24),

$$
\left(\frac{d^{2}}{d r^{2}}-m_{\mathrm{s}}^{2}\right)\left(r \phi_{\mathrm{o}}^{\left(n^{\prime}+1\right)}\right)=r\left\{-g_{\mathrm{s}} \rho_{\mathrm{s}}\left[\rho_{q}^{(n)}, \phi_{0}^{\left(n^{\prime}\right)}\right] \xi^{\left(n^{\prime}\right)}+\delta^{\left(n^{\prime}\right)}\right\}
$$

The new index $n^{\prime}$ indicates that between the steps $n$ and $n+1$, Eq. (4.40) has to be iterated until consistency in $\phi_{0}^{(n+1)}$ is achieved. The presence of second order derivatives of $\phi_{\mathrm{o}}$ in $\rho_{\mathrm{s}}$ can make this condition difficult to fulfil (this point will be discussed below). Note that in (4.40) the scalar density $\rho_{\mathrm{s}}$ is evolved without changing $\rho_{q}^{(n)}$. The boundary conditions $r \phi_{0}=0$ at the origin and at the infinite mesh point are imposed. We have found that the results are quite alike if instead, vanishing slope of $\phi_{0}$ is chosen at the mesh boundaries.

The potentials $A_{0}^{(n+1)}, b_{0}^{(n+1)}$ and $V_{0}^{(n+1)}$ are generated by similar techniques. Now the process only requires one elimination in Eqs. (4.21)-(4.23) because the source terms do not contain the fields. Of course, the appropiate boundary condition for the Coulomb potential at the infinity is $4 \pi r A_{0}=Z e$, where $Z$ is the proton number.

(iii) With the new potentials compute $h_{\mathrm{ETF}}^{(n+1 / 2)}$ and obtain $\Phi_{q}^{(n+1)}$ according to (4.39), with $\Phi_{q}$ subject to the boundary condition that $d \Phi_{q} / d r$ vanishes at the origin and at the mesh edge. Normalize $\rho_{q}^{(n+1)}$ to the particle number. Check numerical convergence.

The steps (ii) and (iii) are then repeated until the desired accuracy is attained. The calculations have been performed on a mesh with a spacing $\Delta r=0.1 \mathrm{fm}$. The r-derivatives have been discretized using seven-point formulae although, in practice, simpler formulae suffice. The parameters $\xi$ and $\Delta \tau$ are not independent and influence critically the rate of convergence. Typical values are $\xi \sim 2.10^{5} \mathrm{MeV}^{2} \mathrm{fm}^{2}$ and $\Delta \tau \sim 2.510^{-23} \mathrm{~s}$. The code runs until the condition

$$
\sum_{i} \frac{\left|\Phi^{(n+1)}\left(r_{i}\right)-\Phi^{(n)}\left(r_{i}\right)\right|}{\Phi^{(n+1)}\left(r_{i}\right)} \leq 10^{-6}
$$

is satisfied (the total energy converges much faster than the densitites).

In the ITS method the chemical potential $\lambda_{q}$ is issued as a by-product and can be used as an additional test of the degree of convergence achieved. We have found that 
$\delta \mathcal{E}^{\mathrm{sc}} / \delta \rho_{q}$ is independent of the position to a few parts in $10^{7}$ when it is computed from the converged densities. Alternatively, the chemical potential can be obtained from the total energy of neighbouring nuclei as $\lambda_{q}=\Delta E / \Delta N_{q}$. We have checked that both methods yield chemical potentials that agree better than $0.1 \%$. Altogether, these controls leave little room for numerical error.

\subsubsection{Results and discussion}

The sets of coupling constants, masses and nuclear matter properties corresponding to the forrces SRK3M5, SRK3M7, ZM, HII and HIV are collected in Table 4.1. When the parametrization includes a $\rho$ meson (SRK3M5, SRK3M7 and ZM), we have adjusted the coupling constant $g_{\rho}$ to approximately reproduce the experimental binding energy of ${ }^{208} \mathrm{~Pb}$ in the Hartree approximation. To compare the energies calculated in the present work with the experimental values, one has to add to them the centre-of-mass correction $-E_{\text {kin }} / A$, where $E_{\text {kin }}$ is the corresponding total kinetic energy, as it is usually done in non-relativistic calculations [Be75, Fl75].

The different approximations will be labelled in the following way. Semiclassical results comprise two cases: $\hbar^{0}$-order Thomas-Fermi calculations (TF $\hbar^{0}$ ), and solutions which include $\hbar^{2}$ corrections $\left(\mathrm{TF} \hbar^{2}\right)$. The quantal results have been obtained from self-consistent Hartree calculations ${ }^{\dagger}$.

In the non-relativistic case, a simple way of incorporating shell effects into the semiclassical calculation consists in adding them perturbatively, i.e., the Hartree-Fock equations are iterated only once employing the self-consistent $\mathrm{TF} \hbar^{2}$ potentials and then use is made of the wave-functions so obtained to calculate the densities and energies. The results of this so-called expectation value method [Bo76a, Br85] are found to be in good agreement with the quantal ones for spherical closed shell nuclei. The same technique can be applied to the relativistic case: the Dirac-Hartree equations for the nucleons are solved only once with the self-consistent relativistic $\mathrm{TF} \hbar^{2}$ fields and the resulting quantal densities are used to evaluate the quantal fields by solving the corresponding Klein-Gordon equations. Finally, the energy is obtained by means of these densities and fields. We will denote this approach by $\mathrm{H}^{*}$.

As indicated in the preceding subsection, the semiclassical densities and fields are obtained from the set of coupled differential equations (4.20)-(4.24) using the imagi-

The author feels most gratified towards Saturnino Marcos for supplying us with the Hartree results which will be presented in this subsection. 
Table 4.1. Parameters and nuclear matter properties (energy per particle $E / A$, particle density $\rho_{0}$, incompressibility $\kappa$, volume symmetry energy $a_{\text {sym }}=k_{\mathrm{F}}^{2} /\left(6 \varepsilon_{\mathrm{F}}\right)+g_{\rho}^{2} \rho_{0} /\left(8 m_{\rho}^{2}\right)$ and effective mass $m^{*} / m$ at saturation) of the five forces considered in this subsection. We have introduced the quantities $C_{i}{ }^{2}=g_{i}{ }^{2}\left(m / m_{i}\right)^{2}$, with $i=\mathrm{s}, \mathrm{v}, \rho, \bar{b}=b /\left(m g_{\mathrm{s}}^{3}\right)$ and $\bar{c}=c / g_{\mathrm{s}}^{4}$. The masses are given in $\mathrm{MeV}$.

\begin{tabular}{lccccc}
\hline & SRK3M5 & SRK3M7 & ZM & HII & HIV \\
\hline$C_{\mathbf{s}}{ }^{2}$ & 380.792 & 233.239 & 169.200 & 246.068 & 218.231 \\
$C_{\mathrm{v}}{ }^{2}$ & 264.687 & 132.497 & 59.100 & 156.327 & 102.615 \\
$C_{\rho}{ }^{2}$ & 26.645 & 85.645 & 11.395 & $\cdot$ & \\
$\bar{b} \times 10^{3}$ & 1.618 & 3.292 & & 1.8 & 8.95 \\
$\bar{c} \times 10^{3}$ & -2.297 & 3.978 & & 0.287 & 3.689 \\
$m$ & 939 & 939 & 938 & 939 & 939 \\
$m_{\mathbf{s}}$ & 500 & 500 & 420 & 550 & 550 \\
$m_{\mathrm{v}}$ & 783 & 783 & 783 & 783 & 783 \\
$m_{\rho}$ & 763 & 763 & 763 & & \\
$E / A(\mathrm{MeV})$ & -16.0 & -16.0 & -16.0 & -15.85 & -15.97 \\
$\rho_{0}\left(\mathrm{fm}{ }^{-3}\right)$ & 0.150 & 0.150 & 0.160 & 0.170 & 0.145 \\
$\kappa(\mathrm{MeV})$ & 300 & 300 & 225 & 345 & 240 \\
$a_{\text {sym }}(\mathrm{MeV})$ & 23.5 & 28.7 & 15.7 & 17.4 & 13.6 \\
$m^{*} / m$ & 0.55 & 0.75 & 0.85 & 0.68 & 0.80 \\
\hline
\end{tabular}

nary time-step method. A word of caution has to be said about the convergence of the numerical solution of the inhomogeneous Klein-Gordon equation obeyed by the semiclassical scalar field, Eq. (4.24). When the $\hbar^{2}$-order corrections are included, we have not been able in general to find stable solutions for parametrizations which have an effective mass at saturation below $m^{*} / m \approx 0.60$. Rather than a failure of the numerical method we have employed, we consider it an intrinsic instability of the equation itself. We have checked that the term $B_{3 q}\left(k_{\mathrm{F} q}, m^{*}\right) \Delta m^{*}$ arising in the scalar density, Eq. (4.25), originates the numerical instability. This term comes from the variation with respect to $\phi_{0}$ of the correction $B_{3 q}\left(k_{F q}, m^{*}\right)\left(\nabla m^{*}\right)^{2}$ which appears in the energy 
density $\mathcal{E}_{2}$, Eq. (4.14).

To bypass this problem, which we encounter with the parametrization SRK3M5 $\left(m^{*} / m=0.55\right)$, we have splitted the term $B_{3 q}$ in (4.14) by retaining only a fraction $f$ of it in the self-consistent calculation, with $f<1$. The total energy is computed adding perturbatively the remaining $(1-f) B_{3 q}$ part which has been turned off in the minimization procedure. We have found that the Euler-Lagrange equations can be solved if one uses $f \sim 0.5$ and that the perturbative correction is only a few per cent of the total energy in the worst case $\left({ }^{40} \mathrm{Ca}\right.$, parameter set SRK3M5). In fact, based upon experience, we have come to the conclusion that any force with $m^{*} / m \leq 0.60$ whose numerical solution of order $\hbar^{2}$ is not convergent, can be made stable if the value of the scalar mass $m_{\mathrm{s}}$ is conveniently reduced (keeping the ratio $g_{\mathrm{s}}^{2} / m_{\mathrm{s}}^{2}$ fixed so as not to change the nuclear matter properties). The converse operation is also true: wellbehaved TF $\hbar^{2}$ solutions turn unstable if $m_{\mathbf{s}}$ is increased. This is no wonder since a change in $m_{s}$ means a change in $g_{s}$, and thus the strength of the source term in the Klein-Gordon equation for the scalar field is correspondingly decreased or increased.

The total energies, proton and neutron r.m.s. radii calculated in the $\mathrm{TF} \hbar^{0}, \mathrm{TF} \hbar^{2}$, Hartree and $\mathrm{H}^{*}$ approximations with the forces SRK3M5, SRK3M7 and ZM are displayed in Tables 4.2 and 4.3 for ${ }^{40} \mathrm{Ca}$ and ${ }^{208} \mathrm{~Pb}$, respectively. In Tables 4.4 and 4.5 results obtained with the forces HII and HIV are shown for some spherical nuclei. From these tables it is seen that the TF $\hbar^{0}$ solution is less bound than the Hartree one for small values of the effective mass, but becomes more bound than Hartree and TF $\hbar^{2}$ for large values of $m^{*}$. The $\mathrm{TF} \hbar^{2}$ approximation yields more binding than the Hartree

Table 4.2. Total energy (in $\mathrm{MeV}$ ), proton and neutron r.m.s. radii (in $\mathrm{fm}$ ) of ${ }^{40} \mathrm{Ca}$ calculated with the different approaches studied in this paper (the label $\mathrm{H}$ corresponds to the Hartree approximation). The listed energies have not been corrected for the centre-of-mass motion.

\begin{tabular}{|c|c|c|c|c|c|c|c|c|c|}
\hline & \multicolumn{3}{|c|}{ SRK3M5 } & \multicolumn{3}{|c|}{ SRK3M7 } & \multicolumn{3}{|c|}{$\mathrm{ZM}$} \\
\hline & $E$ & $r_{\mathrm{n}}$ & $r_{\mathrm{p}}$ & $E$ & $r_{\mathrm{n}}$ & $r_{\mathrm{p}}$ & $E$ & $r_{\mathbf{n}}$ & $r_{\mathrm{p}}$ \\
\hline $\mathrm{TF} \hbar^{0}$ & -292.3 & 3.40 & 3.46 & -355.2 & 3.19 & 3.22 & -345.0 & 3.17 & 3.23 \\
\hline $\mathrm{TF} \hbar^{2}$ & -318.8 & 3.28 & 3.33 & -348.1 & 3.17 & 3.21 & -321.8 & 3.20 & 3.26 \\
\hline $\mathrm{H}$ & -304.9 & 3.35 & 3.42 & -337.0 & 3.25 & 3.30 & -317.0 & 3.27 & 3.33 \\
\hline $\mathrm{H}^{*}$ & -304.8 & 3.33 & 3.40 & -336.6 & 3.24 & 3.26 & -316.9 & 3.25 & 3.33 \\
\hline
\end{tabular}


Table 4.3. Same as Table 4.2 for ${ }^{208} \mathrm{~Pb}$.

\begin{tabular}{|c|c|c|c|c|c|c|c|c|c|}
\hline & \multicolumn{3}{|c|}{ SRK3M5 } & \multicolumn{3}{|c|}{ SRK3M7 } & \multicolumn{3}{|c|}{$\mathrm{ZM}$} \\
\hline & $E$ & $r_{\mathrm{n}}$ & $r_{\mathrm{p}}$ & $E$ & $r_{\mathrm{n}}$ & $r_{\mathrm{p}}$ & $E$ & $r_{\mathrm{n}}$ & $r_{\mathrm{p}}$ \\
\hline$T F \hbar^{0}$ & -1563.8 & 5.76 & 5.60 & -1697.3 & 5.60 & 5.47 & -1739.0 & 5.48 & 5.46 \\
\hline $\mathrm{TF} \hbar^{2}$ & -1641.2 & 5.67 & 5.51 & -1679.8 & 5.59 & 5.45 & -1677.1 & 5.49 & 5.46 \\
\hline $\mathrm{H}$ & -1620.1 & 5.65 & 5.47 & -1620.1 & 5.63 & 5.44 & -1619.1 & 5.53 & 5.49 \\
\hline $\mathrm{H}^{*}$ & -1615.5 & 5.71 & 5.55 & -1617.2 & 5.64 & 5.47 & -1618.0 & 5.53 & 5.50 \\
\hline
\end{tabular}

approximation, i.e., when the relativistic $\hbar^{2}$ corrections are added the TF $\hbar^{2}$ energies always lie below the Hartree ones. In general, the semiclassical radii are smaller or larger than the corresponding Hartree values depending on whether the binding energies are, respectively, larger or smaller than the Hartree ones.

The discrepancy between $\mathrm{TF} \hbar^{0}$ and Hartree results varies almost linearly as a function of the effective mass, both energies would be roughly the same for $m^{*} / m \approx 0.65$. The situation is illustrated in Fig. 4.1, where the difference of the semiclassical energies to the Hartree ones $\left(E^{\mathrm{sc}}-E^{\mathrm{H}}\right)$ has been plotted as a function of $m^{*} / m$ for the $\hbar^{0}$ and $\hbar^{2}$ approximations. Results are presented for the forces SRK3M5, SRK3M7, ZM

Table 4.4. Total energies $E$ (in $\mathrm{MeV}$ ), neutron r.m.s. radii $r_{\mathfrak{n}}$ (in $\mathrm{fm}$ ) and proton r.m.s. radii $r_{\mathrm{p}}$ (in $\mathrm{fm}$ ) obtained using the parameter set HII. The listed energies have not been corrected for the centre-of-mass motion.

\begin{tabular}{|c|c|c|c|c|c|c|c|c|c|}
\hline & \multicolumn{3}{|c|}{ Hartree } & \multicolumn{3}{|c|}{$\mathrm{TF} \hbar^{2}$} & \multicolumn{3}{|c|}{$\mathrm{TF} \hbar^{0}$} \\
\hline & $E$ & $r_{\mathrm{n}}$ & $r_{\mathrm{p}}$ & $E$ & $r_{\mathrm{n}}$ & $r_{\mathrm{p}}$ & $E$ & $r_{\mathrm{n}}$ & $r_{\mathrm{p}}$ \\
\hline${ }^{40} \mathrm{Ca}$ & -325.9 & 3.10 & 3.17 & -342.6 & 3.00 & 3.05 & -332.0 & 3.06 & 3.11 \\
\hline${ }^{48} \mathrm{Ca}$ & -405.6 & 3.35 & 3.22 & -430.6 & 3.25 & 3.16 & -418.8 & 3.31 & 3.22 \\
\hline${ }^{90} \mathrm{Zr}$ & -752.4 & 4.03 & 4.00 & -793.4 & 3.96 & 3.94 & -774.1 & 4.01 & 4.00 \\
\hline${ }^{116} \mathrm{Sn}$ & -952.6 & 4.38 & 4.35 & -1008.2 & 4.31 & 4.29 & -985.5 & 4.36 & 4.34 \\
\hline${ }^{140} \mathrm{Ce}$ & -1160.3 & 4.66 & 4.61 & -1203.9 & 4.60 & 4.57 & -1178.7 & 4.65 & 4.61 \\
\hline${ }^{208} \mathrm{~Pb}$ & -1629.4 & 5.31 & 5.24 & -1696.5 & 5.27 & 5.23 & -1664.7 & 5.31 & 5.27 \\
\hline
\end{tabular}


Table 4.5. Same as Table 4.4 for the parametrization HIV.

\begin{tabular}{|c|c|c|c|c|c|c|c|c|c|}
\hline & \multicolumn{3}{|c|}{ Hartree } & \multicolumn{3}{|c|}{$\mathrm{TF} \hbar^{2}$} & \multicolumn{3}{|c|}{$\mathrm{TF} \hbar^{0}$} \\
\hline & $E$ & $r_{\mathrm{n}}$ & $r_{\mathrm{p}}$ & $E$ & $r_{\mathrm{n}}$ & $r_{\mathrm{p}}$ & $E$ & $r_{\mathrm{n}}$ & $r_{\mathrm{p}}$ \\
\hline${ }^{40} \mathrm{Ca}$ & -392.3 & 3.20 & 3.27 & -411.7 & 3.13 & 3.18 & -428.3 & 3.14 & 3.19 \\
\hline${ }^{48} \mathrm{Ca}$ & -476.5 & 3.48 & 3.36 & -511.0 & 3.38 & 3.32 & -529.4 & 3.38 & 3.34 \\
\hline${ }^{90} \mathrm{Zr}$ & -868.6 & 4.21 & 4.18 & -923.5 & 4.14 & 4.16 & -950.7 & 4.15 & 4.17 \\
\hline${ }^{116} \mathrm{Sn}$ & -1099.3 & 4.56 & 4.58 & -1169.3 & 4.52 & 4.53 & -1201.2 & 4.53 & 4.55 \\
\hline${ }^{140} \mathrm{Ce}$ & -1334.0 & 4.87 & 4.87 & -1394.4 & 4.83 & 4.84 & -1430.2 & 4.84 & 4.85 \\
\hline${ }^{208} \mathrm{~Pb}$ & -1869.9 & 5.56 & 5.55 & -1970.7 & 5.54 & 5.56 & -2016.9 & 5.55 & 5.58 \\
\hline
\end{tabular}

and HII. Carrying out calculations with parametrizations which have an incompressibility $\kappa=400 \mathrm{MeV}$ and $m^{*} / m=0.55$ or 0.75 [Sh91b], we have checked that this behaviour as a function of $m^{*} / m$ is not significantly affected by the value of the nuclear incompressibility.

If we compare the $\mathrm{TF} \hbar^{0}$ and $\mathrm{TF} \hbar^{2}$ results with the Hartree ones, we can see that in practically all cases the second order corrections reduce the differences between $\mathrm{TF} \hbar^{0}$ and Hartree energies in a sensitive way. For the radii, the improvement is not so obvious nor systematic. Had we considered a force with $m^{*} / m \approx 0.65$, the TF $\hbar^{0}$ results would have been in closer agreement with the Hartree values than the TF $\hbar^{2}$ ones. For instance, this happens with the parametrization HII (see Fig. 4.1) which has $m^{*} / m=0.68$. It does not mean the $\mathrm{TF} \hbar^{0}$ approximation to be better than the $\mathrm{TF} \hbar^{2}$ one. Indeed, semiclassical and quantal calculations must differ in the shell energy. This is a subtle quantity that comes from the difference of two large numbers, and it is not easy to evaluate it correctly. Actually, the shell correction is worse estimated in the $\mathrm{TF} \hbar^{0}$ approach than in the $\mathrm{TF} \hbar^{2}$ one because the semiclassical expansion in powers of $\hbar$ is less converged in the former than in the latter. We thus consider the agreement between $\mathrm{TF} \hbar^{0}$ and Hartree results around $m^{*} / m=0.65$ as fortuitous.

To some extent, the above comments also apply to the phenomenology found in nonrelativistic semiclassical calculations with Skyrme forces. That is, the non-relativistic $\mathrm{TF} \hbar^{2}$ energies lie below the HF ones, and the position of $\mathrm{TF} \hbar^{0}$ relative to $\mathrm{TF} \hbar^{2}$ and $\mathrm{HF}$ strongly depends on the effective mass of the force. We have performed calculations for several parametrizations of the Skyrme force. For SV, SIV and SII [Be75], which 


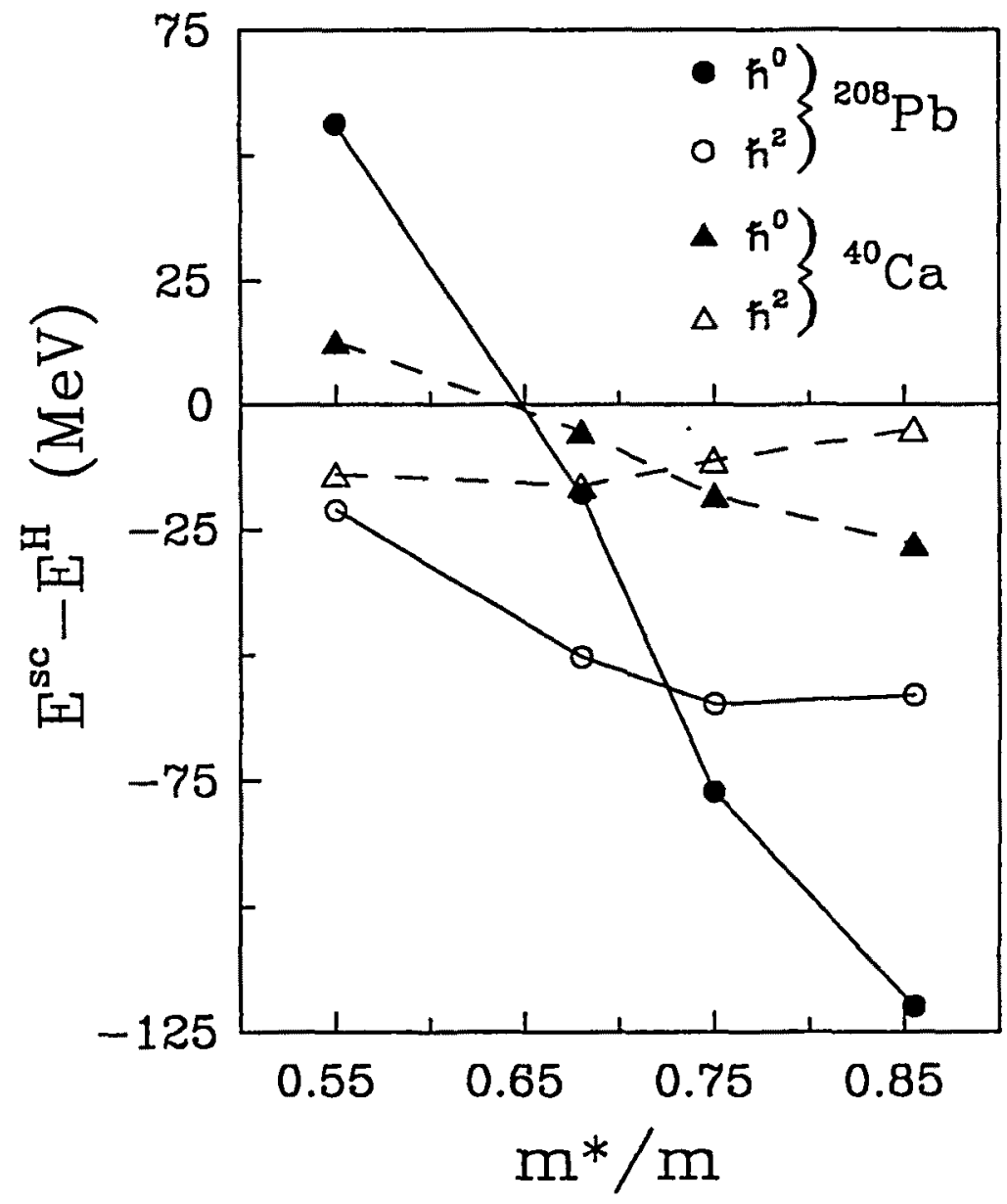

Figure 4.1. Difference between the semiclassical and Hartree energies $\left(E^{\mathrm{sc}}-E^{\mathrm{H}}\right)$ as a function of $m^{*} / m$, in the $\mathrm{TF} \hbar^{0}$ and $\mathrm{TF} \hbar^{2}$ approximations, for ${ }^{208} \mathrm{~Pb}$ (circles) and ${ }^{40} \mathrm{Ca}$ (triangles). The lines connecting the symbols are only to guide the eye.

have $m^{*} / m=0.38,0.47$ and 0.58 respectively, we have found that the TF $\hbar^{0}$ binding energies are smaller than the HF ones. As the effective mass increases, TF $\hbar^{0}$ yields more binding, with binding energies which lie between the HF and TF $\hbar^{2}$ ones for $\mathrm{SkM}^{*}$ $\left(m^{*} / m=0.79\right)[\mathrm{Ba} 82, \mathrm{Br} 85]$ and are larger than the TF $\hbar^{2}$ ones when the effective mass is close to unity (SVI [Be75] or T6 [Ra82]). Results of the energies and radii obtained with the parametrizations SII, SkM* and SVI for ${ }^{40} \mathrm{Ca}$ and ${ }^{208} \mathrm{~Pb}$ are listed, respectively, in Tables 4.6 and 4.7.

Tables 4.2 and 4.3 also show that the perturbative treatment of the shell effects $\left(\mathrm{H}^{*}\right)$ starting from the semiclassical TF $\hbar^{2}$ fields is a good approximation to the full 
Table 4.6. Total energy (in $\mathrm{MeV}$ ), proton and neutron r.m.s. radii (in $\mathrm{fm}$ ) of ${ }^{40} \mathrm{Ca}$ calculated with the non-relativistic Skyrme forces SII $\left(m^{*} / m=0.58\right), \mathrm{SkM}^{*}\left(m^{*} / m=0.79\right)$ and SVI $\left(m^{*} / m=0.95\right)$. The listed energies have not been corrected for the centre-of-mass motion.

\begin{tabular}{|c|c|c|c|c|c|c|c|c|c|}
\hline & \multicolumn{3}{|c|}{ SII } & \multicolumn{3}{|c|}{$\mathrm{SkM}^{*}$} & \multicolumn{3}{|c|}{ SVI } \\
\hline & $E$ & $r_{n}$ & $r_{\mathrm{p}}$ & $E$ & $r_{\mathrm{n}}$ & $r_{p}$ & $E$ & $r_{n}$ & $r_{\mathrm{p}}$ \\
\hline $\mathrm{TF} \hbar^{0}$ & -310.5 & 3.38 & 3.41 & -341.9 & 3.35 & 3.38 & -364.5 & 3.28 & 3.31 \\
\hline $\mathrm{TF} \hbar^{2}$ & -342.9 & 3.28 & 3.30 & -348.7 & 3.25 & 3.29 & -354.7 & 3.26 & 3.29 \\
\hline $\mathrm{H}$ & -326.4 & 3.37 & 3.41 & -325.1 & 3.40 & 3.45 & -324.7 & 3.38 & 3.43 \\
\hline
\end{tabular}

self-consistent quantal calculation, as it happens in the non-relativistic context [Bo76a]. The evaluation of $\mathrm{H}^{*}$ from the $\mathrm{TF} \hbar^{0}$ fields leads, in general, to an agreement in the energies only slightly poorer than when the input are the $\mathrm{TF} \hbar^{2}$ fields, the major discordances (1\%) have been found for SRK3M5. However, starting from the TF $\hbar^{0}$ fields, the results are considerably worse for the radii.

To ascertain the importance of the different terms of the semiclassical functional in the TF $\hbar^{2}$ case, it is useful to discuss in some detail the different contributions to the total energy. Tables 4.8 and 4.9 show, for ${ }^{40} \mathrm{Ca}$ and ${ }^{208} \mathrm{~Pb}$ respectively, the contributions $E_{0}$ and $E_{2}$ to the energy. These terms correspond to the integration over the space of the energy densities $\mathcal{E}_{0}$ and $\mathcal{E}_{2}$, Eqs. (4.13) and (4.14), which are obtained from the solutions of the $\hbar^{2}$-order Euler-Lagrange equations (4.20)-(4.24). The same tables collect the corresponding contributions $T_{0}$ and $T_{2}$ to the kinetic energy. It is seen that the importance of the gradient corrections to the kinetic energy $\left(T_{2}\right)$ and to some parts of $E_{2}$ decreases when $m^{*}$ increases, since the gradients of $m^{*}$ are more important

Table 4.7. Same as Table 4.6 for ${ }^{208} \mathrm{~Pb}$.

\begin{tabular}{|c|c|c|c|c|c|c|c|c|c|}
\hline & \multicolumn{3}{|c|}{ SII } & \multicolumn{3}{|c|}{$\mathrm{SkM}^{*}$} & \multicolumn{3}{|c|}{ SVI } \\
\hline & $E$ & $r_{\mathrm{n}}$ & $r_{\mathrm{p}}$ & $E$ & $r_{\mathrm{n}}$ & $r_{\mathrm{p}}$ & $E$ & $r_{\mathrm{n}}$ & $r_{\mathrm{P}}$ \\
\hline $\mathrm{TF} \hbar^{0}$ & -1534.2 & 5.72 & 5.55 & -1629.4 & 5.65 & 5.52 & -1701.6 & 5.60 & 5.55 \\
\hline $\mathrm{TF} \hbar^{2}$ & -1626.0 & 5.64 & 5.50 & -1652.1 & 5.58 & 5.45 & -1679.4 & 5.59 & 5.53 \\
\hline $\mathrm{H}$ & -1575.9 & 5.69 & 5.50 & -1618.0 & 5.63 & 5.46 & -1618.8 & 5.65 & 5.54 \\
\hline
\end{tabular}


Table 4.8. Contributions (in $\mathrm{MeV}$ ) $E_{0}$ and $E_{2}$ to the total energy of ${ }^{40} \mathrm{Ca}$, as well as their partial decomposition. Also displayed are the corresponding contributions to the kinetic energy $\left(T_{0}, T_{2}\right)$.

\begin{tabular}{lrrr}
\hline & SRK3M5 & SRK3M7 & \multicolumn{1}{c}{ ZM } \\
\hline$E_{0}$ & -10807.3 & -6557.6 & -3361.3 \\
$E_{2}$ & -36.5 & 2.2 & 19.2 \\
$E_{2}\left[(\nabla \rho)^{2}\right]$ & 32.6 & 38.5 & 32.9 \\
$E_{2}\left[\left(\nabla \rho \cdot \nabla m^{*}\right)\right]$ & -46.3 & -29.9 & -12.5 \\
$E_{2}\left[\left(\nabla m^{*}\right)^{2}\right]$ & -22.8 & -6.4 & -1.2 \\
$T_{0}$ & 430.4 & 655.1 & 672.9 \\
$T_{2}$ & 87.8 & 48.1 & 33.4 \\
\hline
\end{tabular}

for small effective masses. It is interesting to note that around $m^{*} / m=0.75$ the $E_{2}$ contribution vanishes. This is due to the fact that in $E_{2}$ the corrections involving $\nabla m^{*}$ have the opposite sign that the $(\nabla \rho)^{2}$ correction. Again, this behaviour is not sensitive to the value of the incompressibility. A similar situation is met in non-relativistic calculations with Skyrme forces: the $\hbar^{2}$ corrections, which include effective mass and spin-orbit contributions, are negative for small and medium $\mathrm{m}^{*}$, becoming positive for $m^{*} / m \geq 0.90$ (forces SVI and T6 for example).

Table 4.9. Same as Table 4.8 for ${ }^{208} \mathrm{~Pb}$.

\begin{tabular}{lrrr}
\hline & SRK3M5 & SRK3M7 & \multicolumn{1}{c}{ ZM } \\
\hline$E_{0}$ & -61956.7 & -35773.2 & -19284.9 \\
$E_{2}$ & -98.8 & 5.4 & 51.1 \\
$E_{2}\left[(\nabla \rho)^{2}\right]$ & 87.7 & 101.9 & 89.7 \\
$E_{2}\left[\left(\nabla \rho \cdot \nabla m^{*}\right)\right]$ & -126.2 & -79.6 & -35.2 \\
$E_{2}\left[\left(\nabla m^{*}\right)^{2}\right]$ & -60.3 & -16.9 & -3.4 \\
$T_{0}$ & 2386.1 & 3570.9 & 3813.9 \\
$T_{2}$ & 230.9 & 127.4 & 91.3 \\
\hline
\end{tabular}


Table 4.10. Kinetic, Coulomb and potential energies coming from the exchange of $\sigma, \omega$ and $\rho$ mesons for ${ }^{40} \mathrm{Ca}$ obtained with the different approaches discussed in the text (the label $\mathrm{H}$ corresponds to the Hartree approximation). The sum of the figures in each column gives the corresponding total energies listed in Table 4.2. All quantities are in MeV.

\begin{tabular}{|c|c|c|c|c|c|c|c|c|c|c|c|c|}
\hline & \multicolumn{4}{|c|}{ SRK3M5 } & \multicolumn{4}{|c|}{ SRK3M7 } & \multicolumn{4}{|c|}{$\mathrm{ZM}$} \\
\hline & $\mathrm{TF} \hbar^{0}$ & $\mathrm{TF} \hbar^{2}$ & $\mathrm{H}$ & $\mathrm{H}^{*}$ & $\mathrm{TF} \hbar^{0}$ & $\mathrm{TF} \hbar^{2}$ & $\mathrm{H}$ & $\mathrm{H}^{*}$ & $\mathrm{TF} \hbar^{0}$ & $\mathrm{TF} \hbar^{2}$ & $\mathrm{H}$ & $\mathrm{H}^{*}$ \\
\hline$E_{\text {kin }}$ & 465.8 & 518.2 & 460.0 & 471.0 & 652.8 & 703.2 & 652.9 & 665.3 & 688.1 & 706.3 & 673.7 & 679.8 \\
\hline$E_{\text {Coul }}$ & 77.9 & 81.1 & 79.7 & 80.1 & 83.4 & 84.0 & 82.4 & 82.9 & 83.3 & 82.8 & 81.5 & 81.8 \\
\hline$E_{\sigma} \times 10^{-2}$ & -51.3 & -57.6 & -55.3 & -56.0 & -37.4 & -38.5 & -36.5 & -37.1 & -23.0 & -22.7 & -22.0 & -22.2 \\
\hline$E_{\omega} \times 10^{-2}$ & 42.9 & 48.4 & 46.8 & 47.4 & 26.5 & 27.2 & 25.7 & 26.2 & 11.8 & 11.6 & 11.3 & 11.4 \\
\hline$\underline{E_{\rho} \times 10^{2}}$ & 5.1 & 4.2 & 4.7 & 4.4 & 8.4 & 7.8 & 9.5 & 9.0 & 3.4 & 3.2 & 3.7 & 3.4 \\
\hline
\end{tabular}

The way the energy is distributed among the various contributions is displayed in Tables 4.10 and 4.11 for ${ }^{40} \mathrm{Ca}$ and ${ }^{208} \mathrm{~Pb}$, respectively. The dominant contributions are those of the $\sigma$ and $\omega$ mesons, although there is a strong cancellation between them. The contribution of the $\rho$ meson plays only a minor role in ${ }^{40} \mathrm{Ca}$ but it is not negligible for ${ }^{208} \mathrm{~Pb}$, all its semiclassical estimates lying close to the quantal result.

It is worth noting that the good agreement in the total energy between the $\mathrm{H}^{*}$ and Hartree approximations is not achieved term by term; it results from compensatory effects and is better for parametrizations having larger $m^{*}$. On the other hand, the

Table 4.11. Same as Table 4.10 for ${ }^{208} \mathrm{~Pb}$. The sum of the figures in each column gives the corresponding total energies listed in Table 4.3.

\begin{tabular}{|c|c|c|c|c|c|c|c|c|c|c|c|c|}
\hline & \multicolumn{4}{|c|}{ SRK3M5 } & \multicolumn{4}{|c|}{ SRK3M7 } & \multicolumn{4}{|c|}{$Z M$} \\
\hline & $T F \hbar^{0}$ & $\mathrm{TF} \hbar^{2}$ & $\mathrm{H}$ & $\mathrm{H}^{*}$ & $T F \hbar^{0}$ & $\mathrm{TF} \hbar^{2}$ & $\mathrm{H}$ & $\mathrm{H}^{*}$ & $\mathrm{TF} \hbar^{0}$ & $T F \hbar^{2}$ & $\mathrm{H}$ & $\mathrm{H}^{*}$ \\
\hline$E_{\text {kin }}$ & 2491. & 2617. & 2479. & 2591. & 3567. & 3698. & 3617. & 3628. & 3855. & 3905 & 3855 . & 3859 \\
\hline$E_{\text {Coul }}$ & 805.8 & 818.8 & 826.4 & 813.2 & 824.3 & 827.7 & 829.4 & 824.8 & 825.7 & 826.1 & 823.8 & 821.6 \\
\hline$E_{\sigma} \times 10^{-3}$ & -30.9 & -32.7 & -33.0 & -32.0 & -20.7 & -21.0 & -20.4 & -20.4 & -13.1 & -13.0 & -12.8 & -12.8 \\
\hline$E_{\omega} \times 10^{-3}$ & 26.0 & 27.6 & 28.1 & 27.0 & 14.5 & 14.7 & 14.3 & 14.2 & 6.7 & 6.6 & 6.5 & 6.5 \\
\hline$\underline{E_{\rho}}$ & 24.5 & 25.8 & 25.5 & 25.4 & 89.0 & 88.4 & 82.4 & 84.7 & 13.7 & 13.4 & 13.1 & 13.2 \\
\hline
\end{tabular}




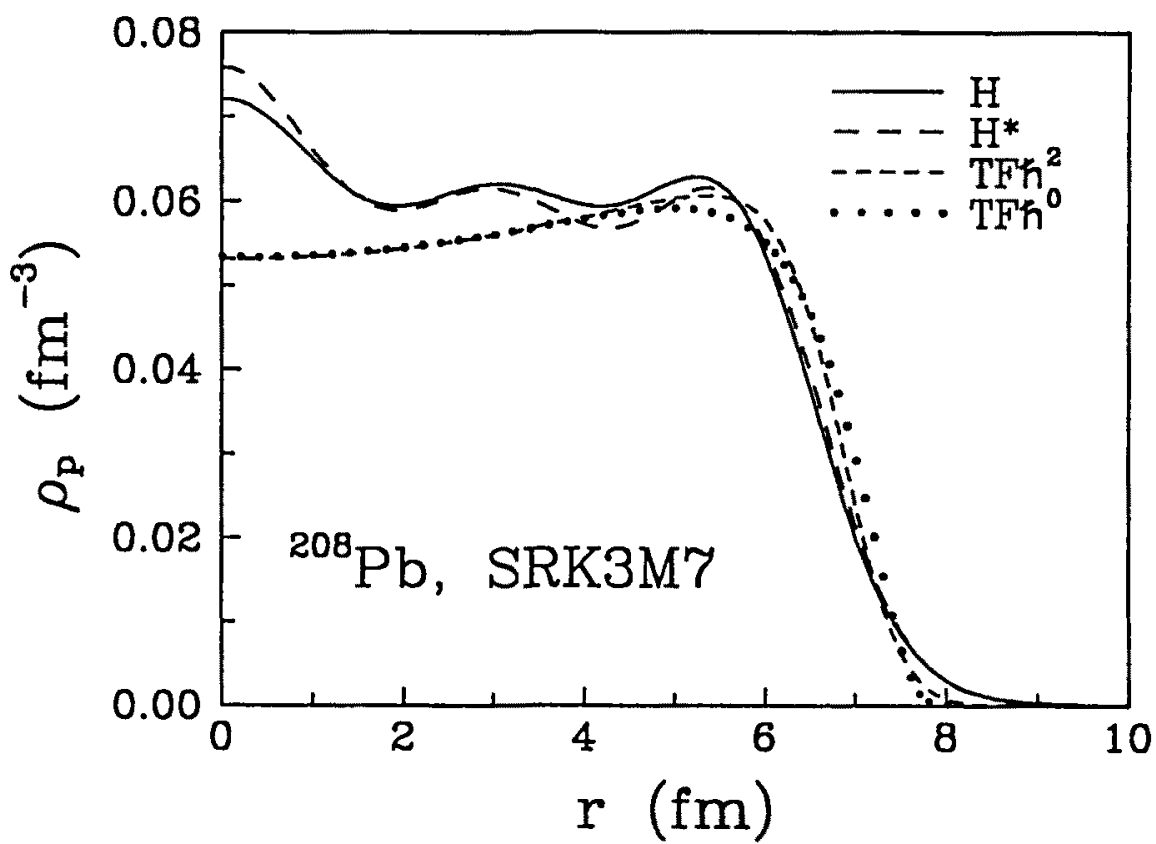

Figure 4.2. Proton density of ${ }^{208} \mathrm{~Pb}$ obtained with the parametrization SRK3M7 in the Hartree (solid line), $\mathrm{H}^{*}$ (long-dashed), $\mathrm{TF} \hbar^{2}$ (short-dashed) and $\mathrm{TF} \hbar^{0}$ (dotted) approximations.

differences between Hartree and the semiclassical methods $\left(\mathrm{TF} \hbar^{0}\right.$ and $\left.\mathrm{TF} \hbar^{2}\right)$ in the partial contributions to the energy are rather large, and also compensatory effects appear in the final result. The situation is similar in the non-relativistic case if we compare the different contributions to the total energy evaluated semiclassically with the corresponding HF contributions (cf. Table 10 of [Ce90a]). Indeed, because of the shell effects present in the Hartree or HF results, there is no reason for these partial quantities to be the same in the quantal and semiclassical approaches.

As a representative example, the proton density of ${ }^{208} \mathrm{~Pb}$ calculated with SRK3M7 in the four approaches considered has been plotted in Fig. 4.2. The results obtained for neutron and scalar densities exhibit similar trends. We notice the good agreement between the Hartree and the $\mathrm{H}^{*}$ densities. The shell effects are rather well incorporated by $\mathrm{H}^{*}$ in the internal region of the densities, which become almost identical in both approximations in the surface and tail.

To complement this picture, Figs. 4.3 and 4.4 show the radial distribution of the proton densities of ${ }^{40} \mathrm{Ca}$ and ${ }^{208} \mathrm{~Pb}$, respectively, calculated in the Hartree and $\mathrm{TF} \hbar^{2}$ 


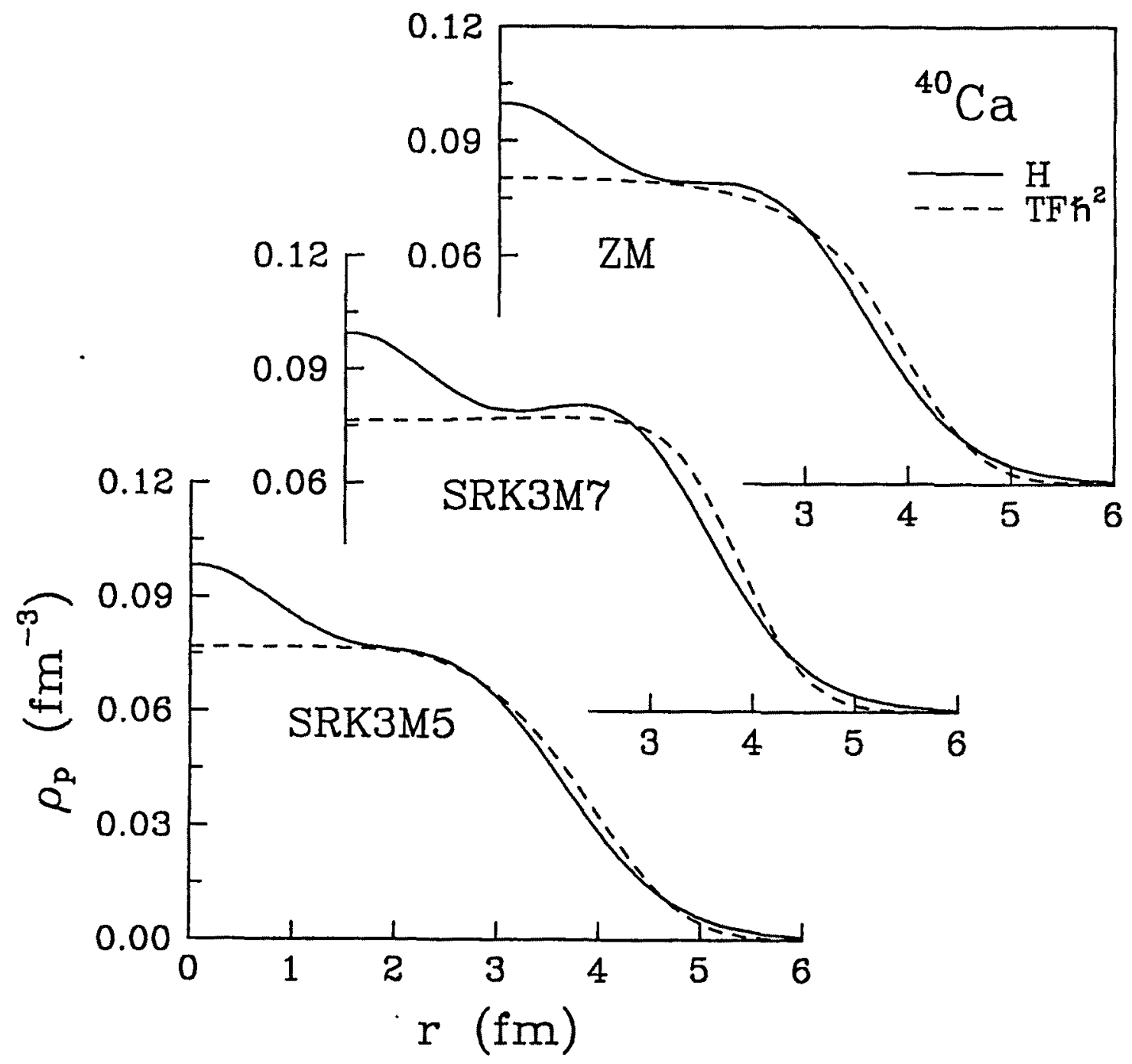

Figure 4.3. Proton density of ${ }^{40} \mathrm{Ca}$, calculated with the forces SRK3M5, SRK3M7 and $\mathrm{ZM}$, in the Hartree $(\mathrm{H})$ and $\mathrm{TF} \hbar^{2}$ approximations.

approximations with the forces SRK3M5, SRK3M7 and ZM. Furthermore, the proton, neutron and scalar densities of ${ }^{208} \mathrm{~Pb}$ obtained in the Hartree, TF $\hbar^{0}$ and $\mathrm{TF} \hbar^{2}$ approximations using the parametrization HII are displayed in Fig. 4.5. The semiclassical $T F \hbar^{0}$ and $T F \hbar^{2}$ density distributions do not present oscillations due to the absence 


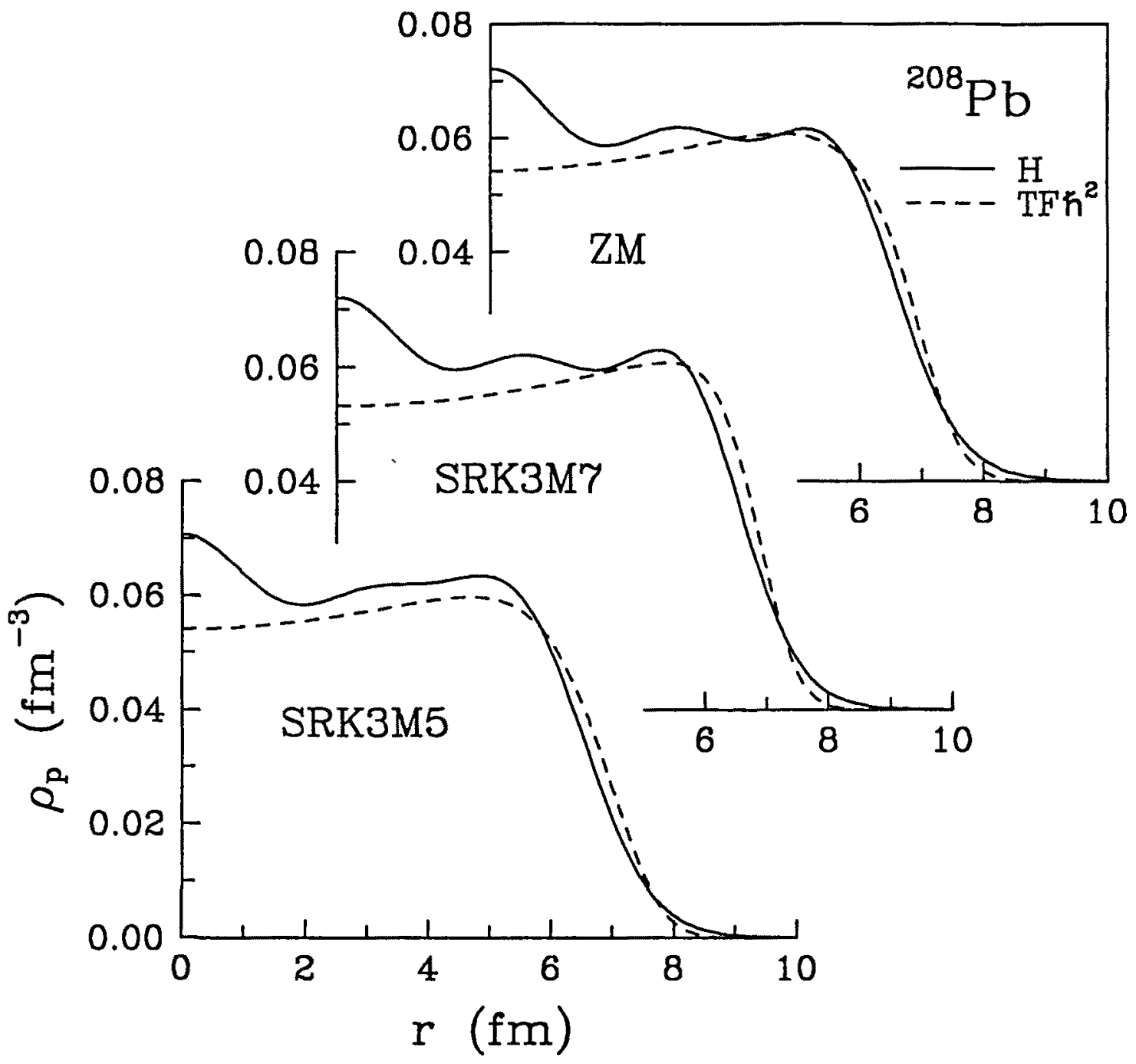

Figure 4.4. Same as Fig. 4.3 for ${ }^{208} \mathrm{~Pb}$.

of shell effects but they average the Hartree results. In the bulk the TF $\hbar^{0}$ and $T F \hbar^{2}$. densities are very similar. However, as expected, the gradient corrections incorporated by the $T F \hbar^{2}$ functionals improve the $\mathrm{TF} \hbar^{0}$ densities at the surface. In this region, the $\mathrm{TF} \hbar^{2}$ densities come closer to the Hartree ones and show a notably better decay than the TF $\hbar^{0}$ densities, despite that the fall-off is still too steep with respect to the Hartree solutions. This is illustrated in Fig. 4.6, which shows a semi-logarithmic plot of the 


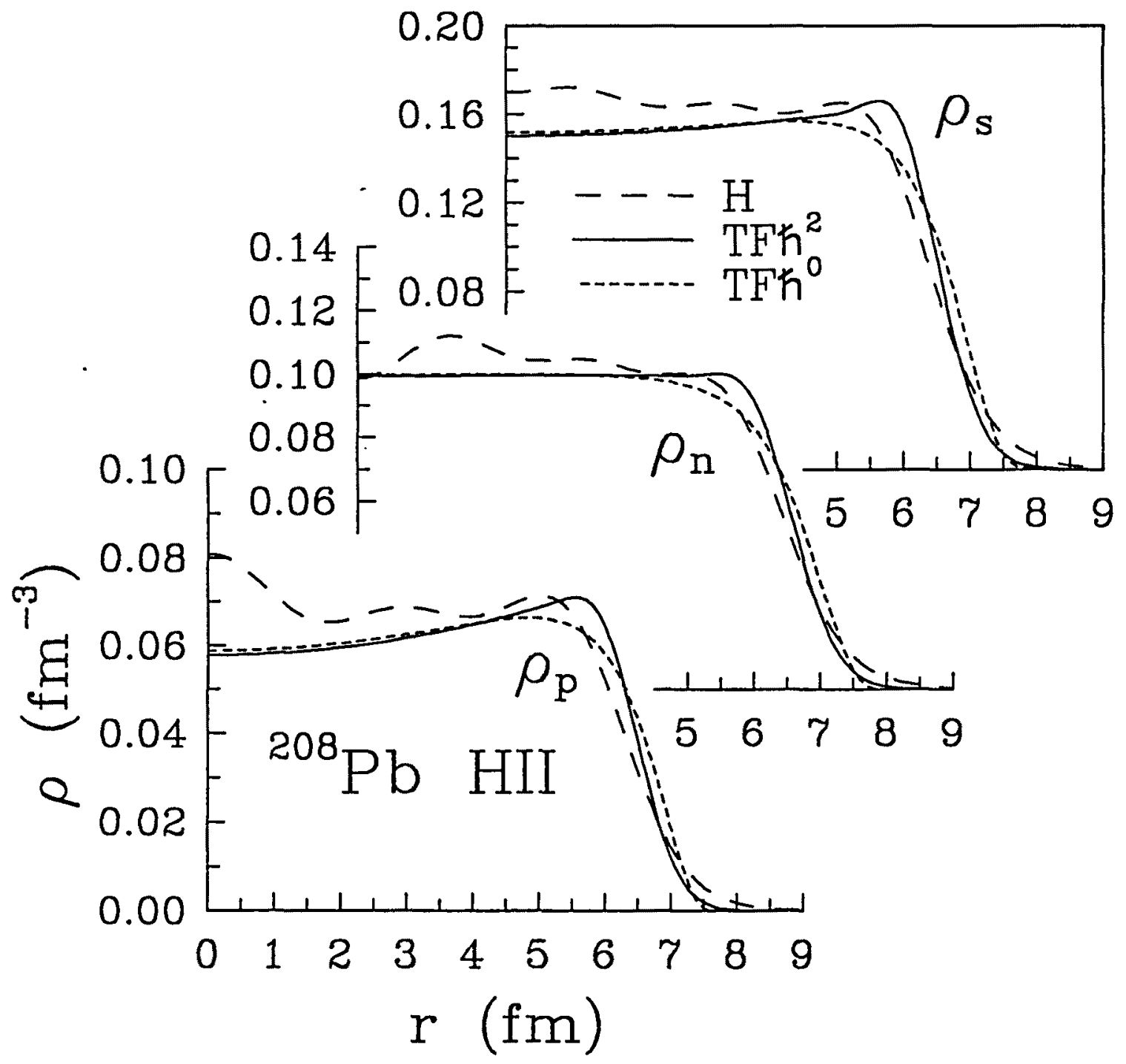

Figure 4.5. Proton, neutron and scalar densities of ${ }^{208} \mathrm{~Pb}$ obtained with the force HII in the Hartree (H), $T F \hbar^{2}$ and $T F \hbar^{0}$ approximations.

proton density of ${ }^{208} \mathrm{~Pb}$ in the outer surface region for SRK3M7.

The comparison between Hartree and $T F \hbar^{2}$ results shows that the situation is quite similar to the one found in the non-relativistic case, see for example Fig. 1 of [Ce90a]. Fig. 4.7 is the equivalent of Fig. 4.2 for the proton kinetic energy density $\tau_{p}$; similar comments to those made for the proton density in Fig. 4.2 apply here. 


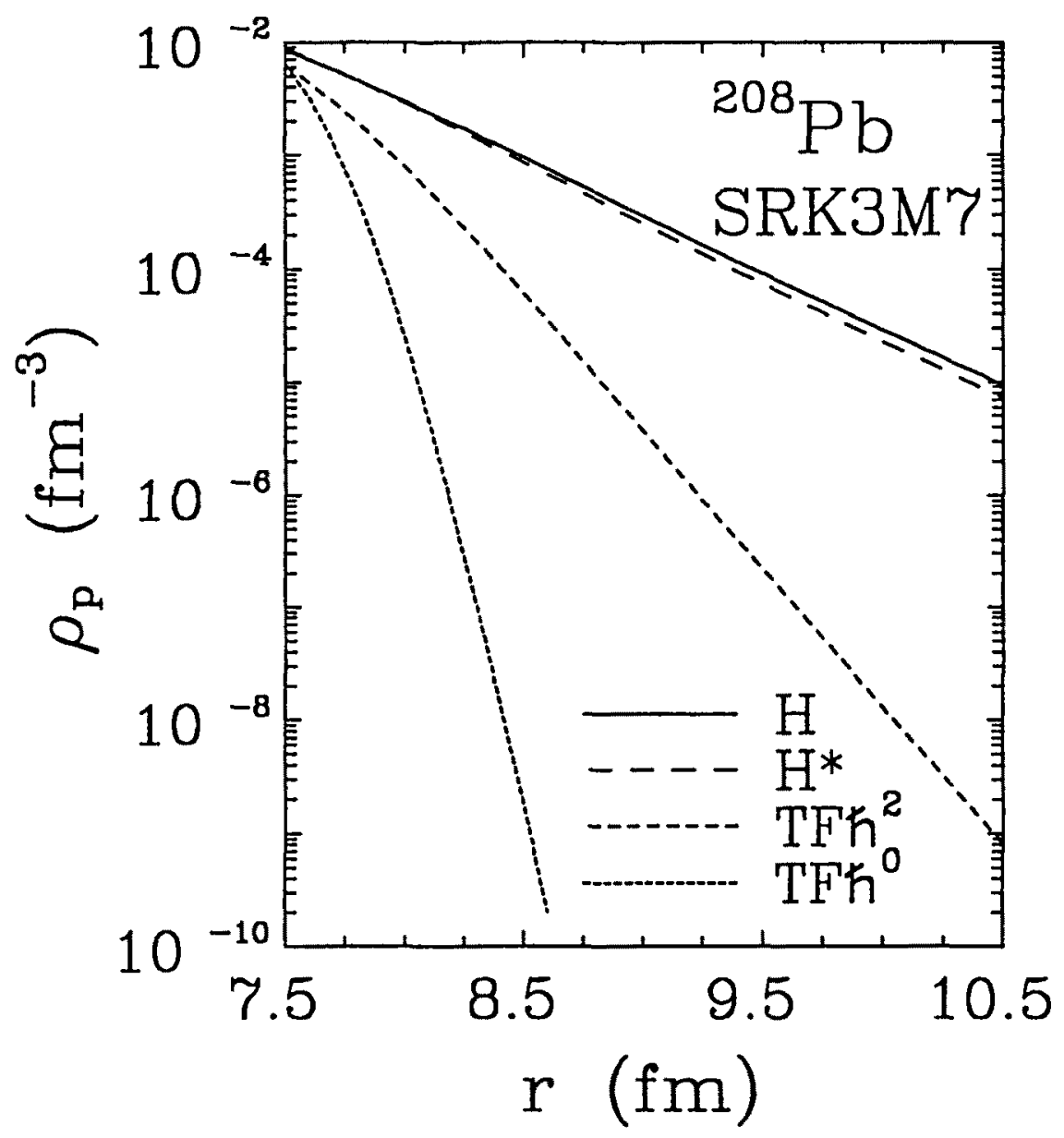

Figure 4.6. Proton density of ${ }^{208} \mathrm{~Pb}$, calculated with SRK3M7, in the outer surface region on a semi-logarithmic scale.

The scalar meson potential $V_{\sigma}=m-m^{*}$ and the vector meson potential $V_{\omega}=g_{\mathrm{v}} V_{0}$ calculated in ${ }^{208} \mathrm{~Pb}$ are displayed in Fig. 4.8 for the forces SRK3M5, SRK3M7 and ZM in the Hartree and TF $\hbar^{2}$ approaches. Notice the change of scale in the vertical axis for each parametrization. Inside the nucleus, the ratio between the vector potentials of any two of these parametrizations is, to a good approximation, equal to the ratio of the corresponding coupling constants $C_{v}^{2}$. For the scalar potential, this is not so because of the non-linear couplings in Eq. (4.24).

In Fig. 4.9 we show the results obtained in lead with HII for the scalar and vector meson potentials $V_{\sigma}$ and $V_{\omega}$ in the $\mathrm{TF} \hbar^{0}, \mathrm{TF} \hbar^{2}$ and Hartree approximations. The 


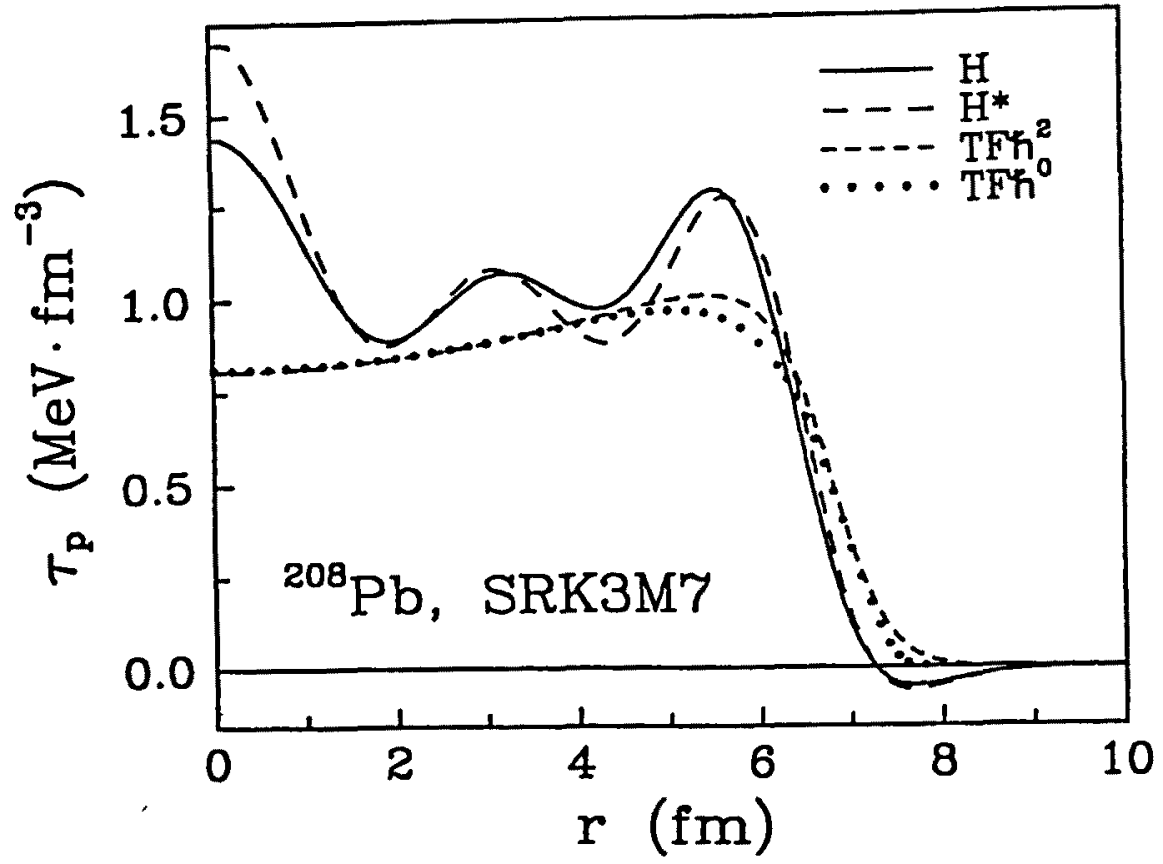

Figure 4.7. Same as Fig. 4.2 for the proton kinetic energy density.

radial dependence of the quantity

$$
\alpha_{\text {s.o. }}=-\frac{1}{2 m^{2} r} \frac{d}{d r}\left(V_{\sigma}+V_{\omega}\right)
$$

which is most sensitive to the nuclear surface, is also shown.

A Foldy-Wouthuysen reduction of the Dirac equation for nucleons moving in the field generated by $V_{\omega}$ and $V_{\sigma}$, transforms it into a Schrödinger equation whose central potential involves $V_{\omega}-V_{\sigma}$ and relates $\alpha_{\text {s.o. }}$ with the strength of the corresponding spin-orbit force [Se86] (i.e., the non-relativistic single-particle effective spin-orbit force depends on the sum $V_{\omega}+V_{\sigma}$, whereas the central term depends on the difference $V_{\omega}-V_{\sigma}$ ). The $\mathrm{TF} \hbar^{2}$ calculation clearly overestimates the maximum value of $\alpha_{\text {s.o. }}$ (Fig. 4.9), but the position of the peak is closer to the Hartree result than with TF $\hbar^{0}$.

Figs. 4.8 and 4.9 show that in the bulk, the central potential is around $-60 \mathrm{MeV}$, deeper for small $m^{*}$, shallower for large $m^{*}$ in accordance with the optical model phenomenology [Bo69]. The changes are far more drastic for the spin-orbit potential, which decreases by a factor of around 3 from SRK3M5 to ZM. This leads to a small splitting in the single particle levels for forces having an effective mass of the order of 0.80 (see also [Ba91, Ko91]), a value compatible with the one extracted from 


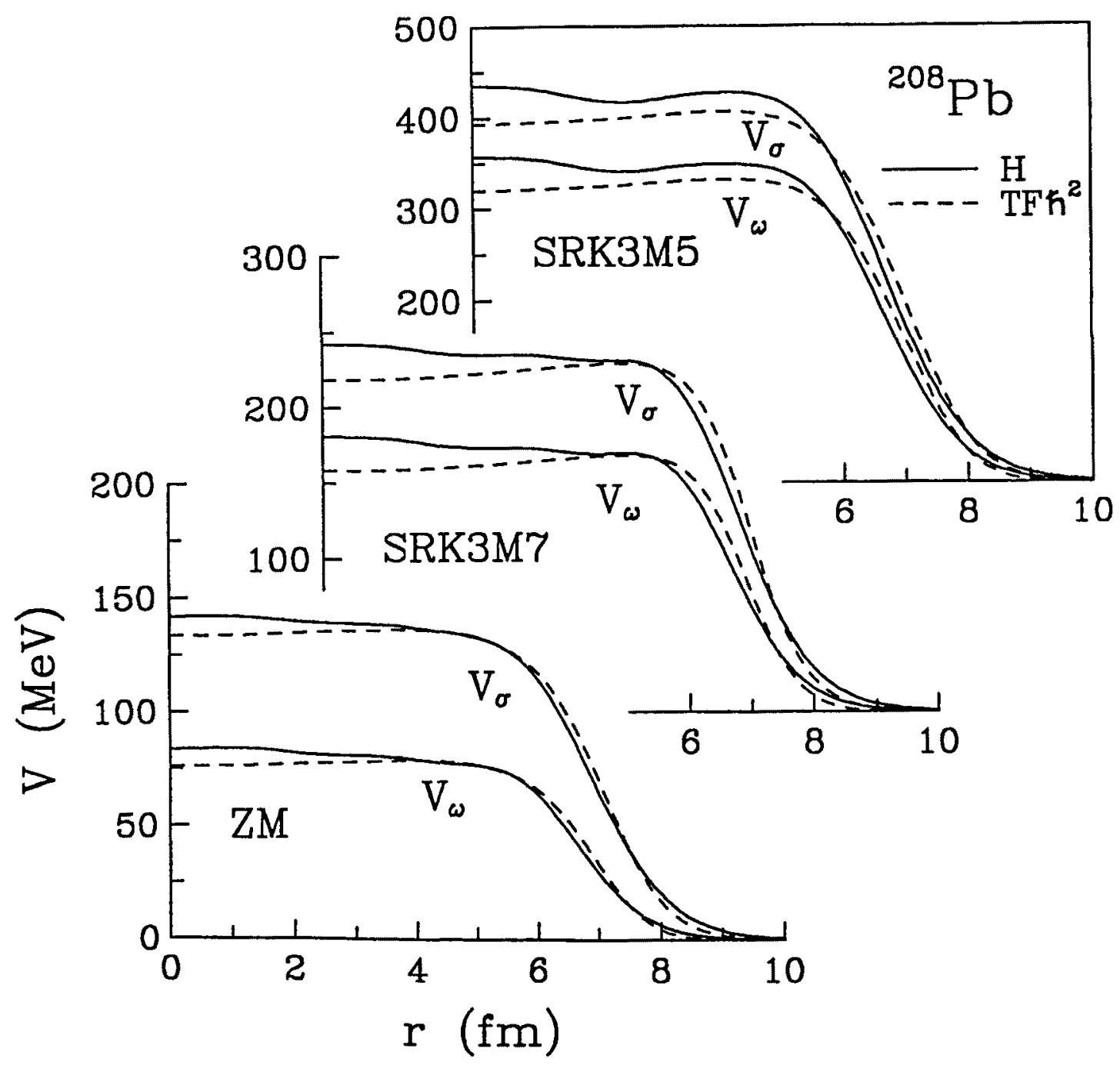

Figure 4.8. Scalar $\left(\mathrm{V}_{\sigma}=m-m^{*}\right)$ and vector $\left(\mathrm{V}_{\omega}=g_{v} V_{0}\right)$ potentials obtained for ${ }^{208} \mathrm{~Pb}$ with the parametrizations SRK3M5, SRK3M7 and ZM. Solid line, Hartree approximation; dashed line, TF $\hbar^{2}$ approximation.

optical model analyses [Jo87]. It seems to be quite a general feature of all the $\sigma-\omega$ Lagrangians that the experimental splitting can only be obtained with rather small effective masses, $m^{*} / m \leq 0.60$, as we stated at the beginning of this chapter.

Finally, let us summarize the two apparent merits which the TF $\hbar^{2}$ approximation 


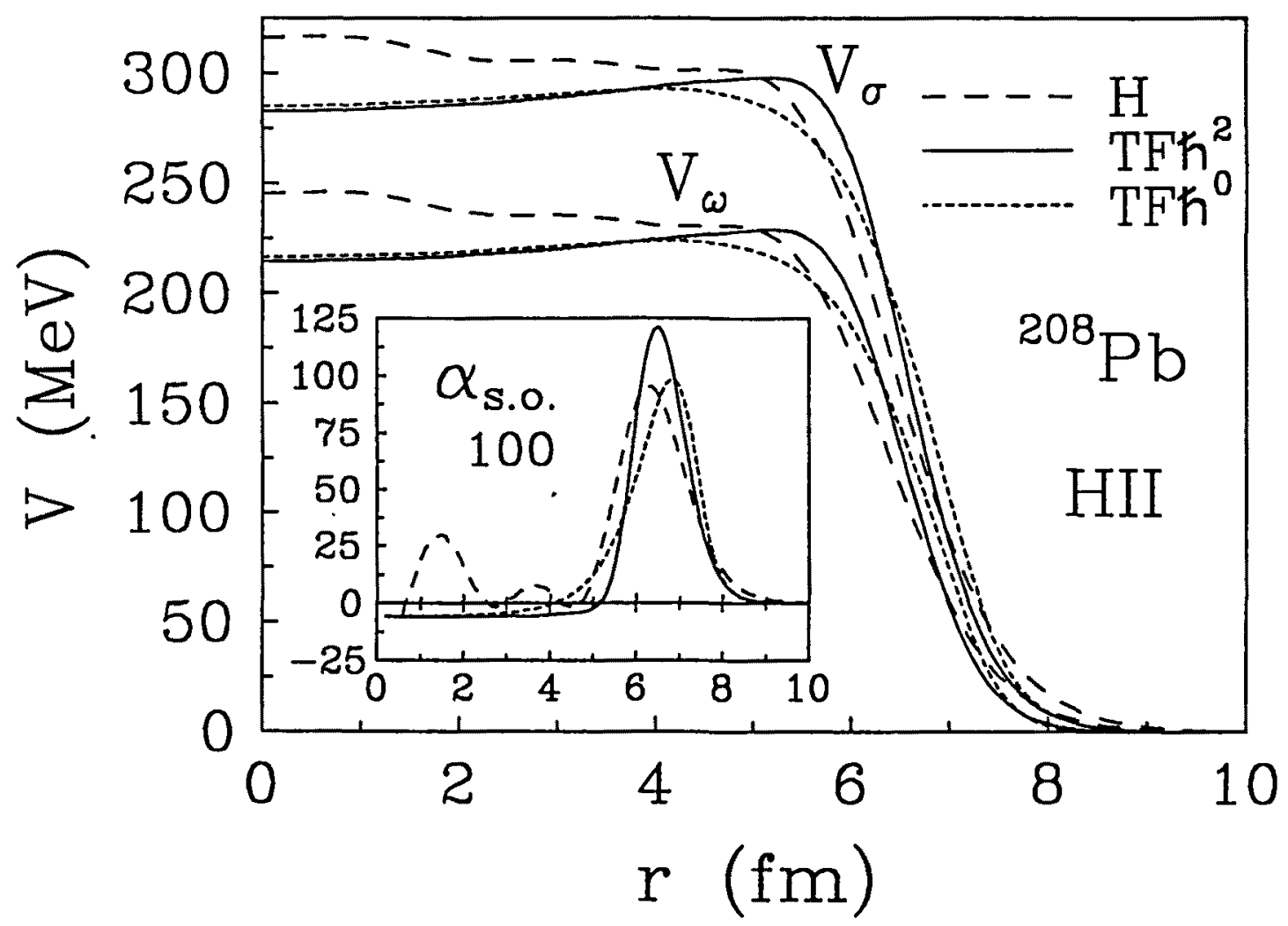

Figure 4.9. Radial dependence of the scalar and vector meson potentials $\left(V_{\sigma}=m-m^{*}\right.$, $\left.V_{\omega}=g_{\mathrm{v}} V_{0}\right)$ and of the quantity $\alpha_{\text {s.o. }}$, Eq. (4.42), calculated in ${ }^{208} \mathrm{pb}$ with HII.

has over the simple $\mathrm{TF} \hbar^{0}$ one. On the one hand, it allows to obtain fully variational densities that go exponentially to zero. On the other hand, it takes into account nonlocal spin-orbit and effective mass contributions up to order $\hbar^{2}$, yielding a more reliable average value. 


\subsection{Liquid Drop Model Coefficients}

The surface energy in relativistic $\sigma-\omega$ models has been estimated by fitting a mass formula to finite nuclei results [Bo77a, Se78, Se79] or calculated from semi-infinite nuclear matter in the Hartree [Bo77b, Ho89] and TF $\hbar^{0}$ [Bo77b, St91, Sh91a, Sh91b] approximations. To our knowledge, no attempt has been made to calculate the curvature coefficient using a relativistic model, and thus merits to be undertaken.

In this section, we shall calculate the semiclassical liquid drop model coefficients related to the surface and curvature energies for some parametrizations of the $\sigma-\omega$ model. We shall use the forces HII and HIV, and also the parameter sets given in Table 4.12: P1 (notation as in [Ho89]) which was fitted to reproduce ground-state properties of finite nuclei [Re86], and PW1 which corresponds to the original linear Walecka model [Se86]. Hartree results of surface properties are available for P1 and PW1 [Ho89].

In the liquid droplet model formulated by Myers and Swiatecki [My69, My74] the

Table 4.12. Parameters and nuclear matter properties of the forces $P 1$ and PW1. Notation as in Table 4.1 .

\begin{tabular}{lcc}
\hline & P1 & PW1 \\
\hline$C_{\mathrm{s}}{ }^{2}$ & 373.176 & 267.568 \\
$C_{\mathrm{v}}^{2}$ & 245.458 & 196.300 \\
$\bar{b} \times 10^{3}$ & 2.48 & \\
$\bar{c} \times 10^{3}$ & -3.50 & \\
$m$ & 938 & 939 \\
$m_{\mathrm{s}}$ & 492.25 & 450 \\
$m_{\mathrm{v}}$ & 795.36 & 783 \\
$E / A(\mathrm{MeV})$ & -16.41 & -15.75 \\
$\rho_{0}\left(\mathrm{fm}{ }^{-3}\right)$ & 0.154 & 0.193 \\
$\kappa(\mathrm{MeV})$ & 205 & 546 \\
$a_{\mathrm{sym}}(\mathrm{MeV})$ & 19.0 & 22.1 \\
$m^{*} / m$ & 0.57 & 0.56 \\
\hline
\end{tabular}


energy of a spherical nucleus is written as:

$$
E=a_{\mathrm{v}} A+4 \pi \int_{0}^{\infty} d r r^{2}\left[\mathcal{E}(r)-a_{\mathrm{v}} \rho(r)\right],
$$

where $a_{v}$ is the energy per particle in infinite nuclear matter and $\mathcal{E}(r)$ and $\rho(r)$ are the energy and particle densities, respectively. Starting from (4.43) and following the method outlined in [My69, My74, St85], one obtains for the surface and curvature coefficients in semi-infinite nuclear matter the following expressions:

$$
\begin{aligned}
a_{\mathrm{s}}= & \left.4 \pi r_{0}^{2} \int_{-\infty}^{\infty} d z\left[\mathcal{E}(z)-a_{\mathrm{v}} \rho(z)\right]\right|_{\kappa=0} \\
a_{\mathrm{c}}=8 \pi r_{0} & {\left[\left.\int_{-\infty}^{\infty} d z\left(z-z_{0}\right)\left[\mathcal{E}(z)-a_{\mathrm{v}} \rho(z)\right]\right|_{\kappa=0}\right.} \\
& \left.\quad+\left.\int_{-\infty}^{\infty} d z \frac{\partial\left[\mathcal{E}(z)-a_{\mathrm{v}} \rho(z)\right]}{\partial \kappa}\right|_{\kappa=0}\right],
\end{aligned}
$$

where $r_{0}=\left[3 /\left(4 \pi \rho_{0}\right)\right]^{1 / 3}$ is the nuclear matter radius, $z_{0}$ is the location of the equivalent sharp surface, and $\kappa$ is the curvature $(2 / R$ for a sphere of radius $R$ ).

The two contributions to the curvature energy in (4.45) are called geometrical and dynamical, respectively. The geometrical contribution only involves the variation of the surface energy density $\mathcal{E}(z)-a_{\mathrm{v}} \rho(z)$ across the surface parallel to the $z$-axis, while the dynamical part corresponds to the variation of the surface energy density when the plane surface is infinitesimally bent. The surface energy density depends on the curvature $\kappa$ in two different ways: one of them is the explicit dependence of $\mathcal{E}(z)$ on the $\Delta$ operator (think for example of the non-relativistic definition of the kinetic energy density [Lo91]) which in the limit of $R \rightarrow \infty$ reads $d^{2} / d z^{2}+\kappa d / d z$. The other one corresponds to the implicit curvature dependence of $\rho(z)$.

In the relativistic $\sigma-\omega$ model, the semiclassical energy density (4.12) is free of explicit $\Delta$ dependences, since they have been removed by partial integration. On the other hand, the implicit curvature dependence of the nuclear density $\rho$ and of the meson fields $V_{0}$ and $\phi_{0}$ does not contribute to the dynamical part of the curvature energy in a self-consistent calculation, because the surface tension of semi-infinite nuclear matter is stationary with respect to changes in the density and in the meson fields. This is a consequence of the so-called $\dot{\sigma}$-theorem [My69, My74, St85] (see also [St91]). As a result, the curvature energy is only given by its geometrical contribution.

Fig. 4.10 displays the $\mathrm{TF} \hbar^{0}$ and $\mathrm{TF} \hbar^{2}$ density profiles of semi-infinite nuclear matter obtained with the HII set, and Figs. 4.11 and 4.12 show the $\mathrm{TF} \hbar^{0}$ and $\mathrm{TF} \hbar^{2}$ surface 


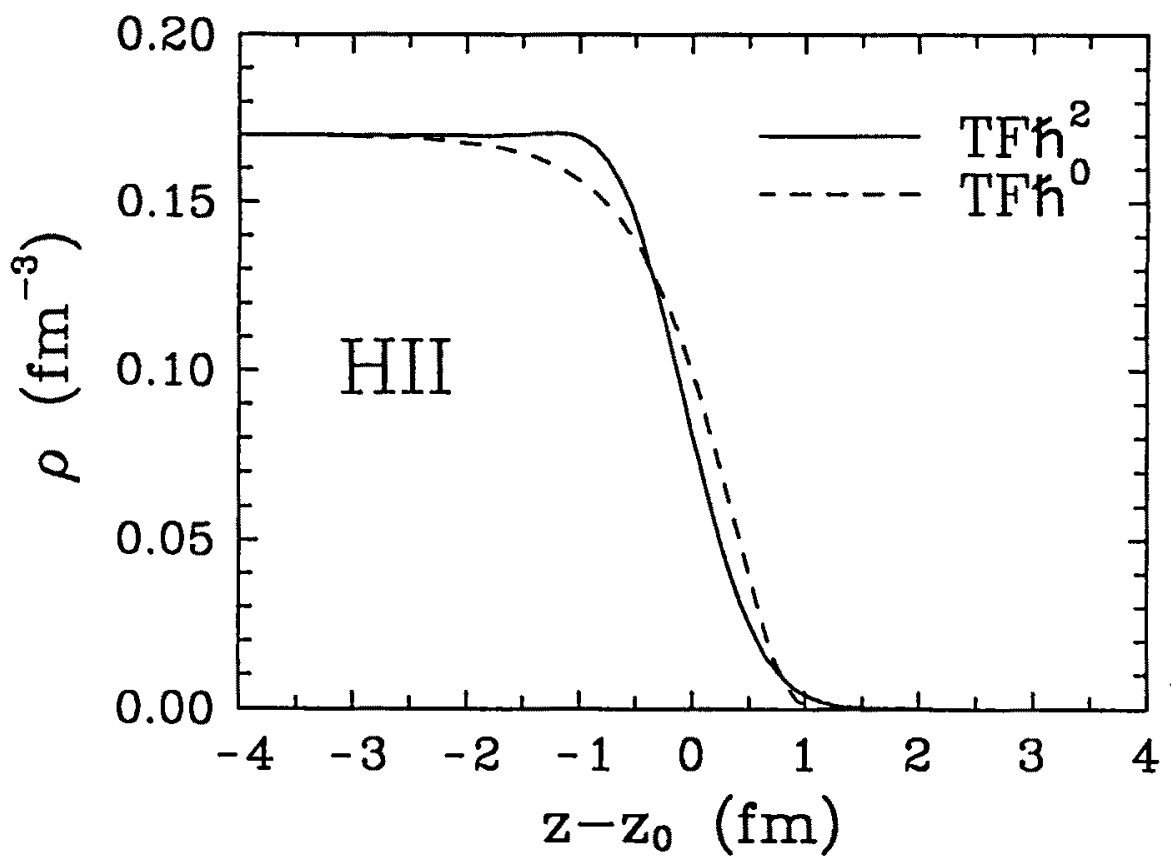

Figure 4.10. Density profile of symmetric semi-infinite nuclear matter obtained with the force $\mathrm{HII}$ in the $\mathrm{TF} \hbar^{2}$ and $\mathrm{TF} \hbar^{0}$ approximations.

and curvature energy densities for HII. Table 4.13 collects the surface and curvature coefficients as well as the surface thickness of the semi-infinite density profile (standard $90 \%$ to $10 \%$ distance) calculated with PW1, P1, HII and HIV. As may be seen from this table, the quantal and semiclassical calculations of the surface coefficient and thickness

Table 4.13. Surface $\left(a_{s}\right)$ and curvature $\left(a_{c}\right)$ energies, and surface thickness $(t)$ corresponding to different $\sigma-\omega$ parametrizations and Thomas-Fermi approximations. Also displayed are some Hartree (H) results (from [Ho89]).

\begin{tabular}{|c|c|c|c|c|c|c|c|c|}
\hline & \multicolumn{3}{|c|}{$a_{\mathrm{s}}(\mathrm{MeV})$} & \multicolumn{3}{|c|}{$t(\mathrm{fm})$} & \multicolumn{2}{|c|}{$a_{c}(\mathrm{MeV})$} \\
\hline & $\overline{\mathrm{H}}$ & $\mathrm{TF} \hbar^{2}$ & $\mathrm{TF} \hbar^{0}$ & $\overline{\mathrm{H}}$ & $\mathrm{TF} \hbar^{2}$ & $T F \hbar^{0}$ & $T F \hbar^{2}$ & $\mathrm{TF} \hbar^{0}$ \\
\hline PW1 & 33.8 & 32.8 & 35.9 & 2.38 & 2.27 & 2.71 & 18.8 & 20.1 \\
\hline P1 & 15.8 & 17.4 & 19.9 & 2.31 & 2.09 & 2.96 & 12.4 & 16.0 \\
\hline HII & & 16.0 & 17.3 & & 1.23 & 1.57 & 7.2 & 7.5 \\
\hline HIV & & 11.1 & 10.0 & & 1.07 & 1.00 & 4.9 & 3.7 \\
\hline
\end{tabular}




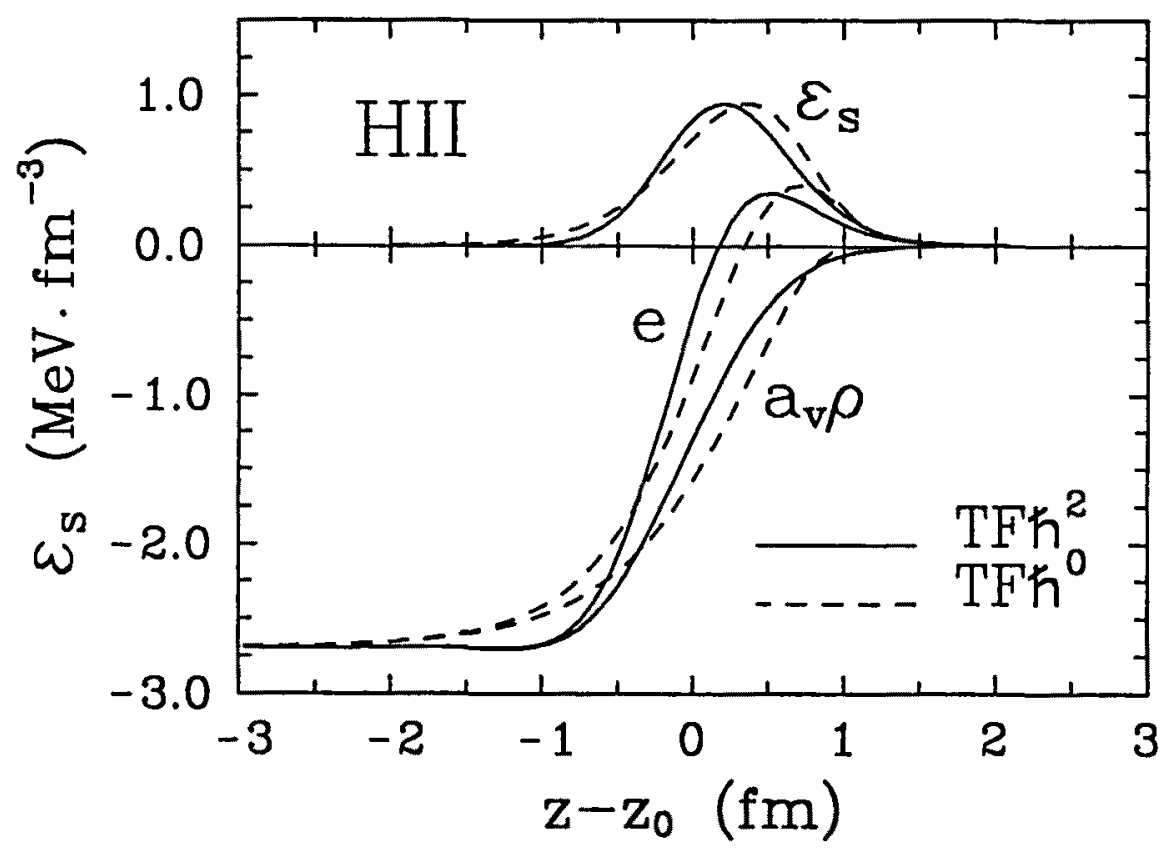

Figure 4.11. Surface energy density $\varepsilon_{s}=\mathcal{E}(z)-a_{\mathrm{v}} \rho(z)$ corresponding to the interaction HII. Also displayed is the separate contribution of the terms $\mathcal{E}(z)$ and $a_{\mathrm{v}} \rho(z)$.

are in reasonable agreement. The values we have found for $a_{c}$ are within the range of those obtained in non-relativistic semiclassical calculations using Skyrme forces [ $\mathrm{Br} 85$, Tr86, St88] (the empirical value is $a_{\mathrm{c}}^{\text {emp }} \approx 0 . \mathrm{MeV}$ [Mö88]). The evolution as a function of the effective mass of the $\mathrm{TF} \hbar^{0}$ results with respect to the $\mathrm{TF} \hbar^{2}$ ones shows the same trend as for finite nuclei.

As discussed by Hofer and Stocker [H089], few parametrizations of the $\sigma-\omega$ model are able to simultaneously yield acceptable values for the surface energy and thickness ( $a_{s}$ should be around 16.5-21 MeV and $t$ around 2.2-2.5 fm). Among the ones used here, only the parametrization P1 gives satisfactory results (Table 4.13). 


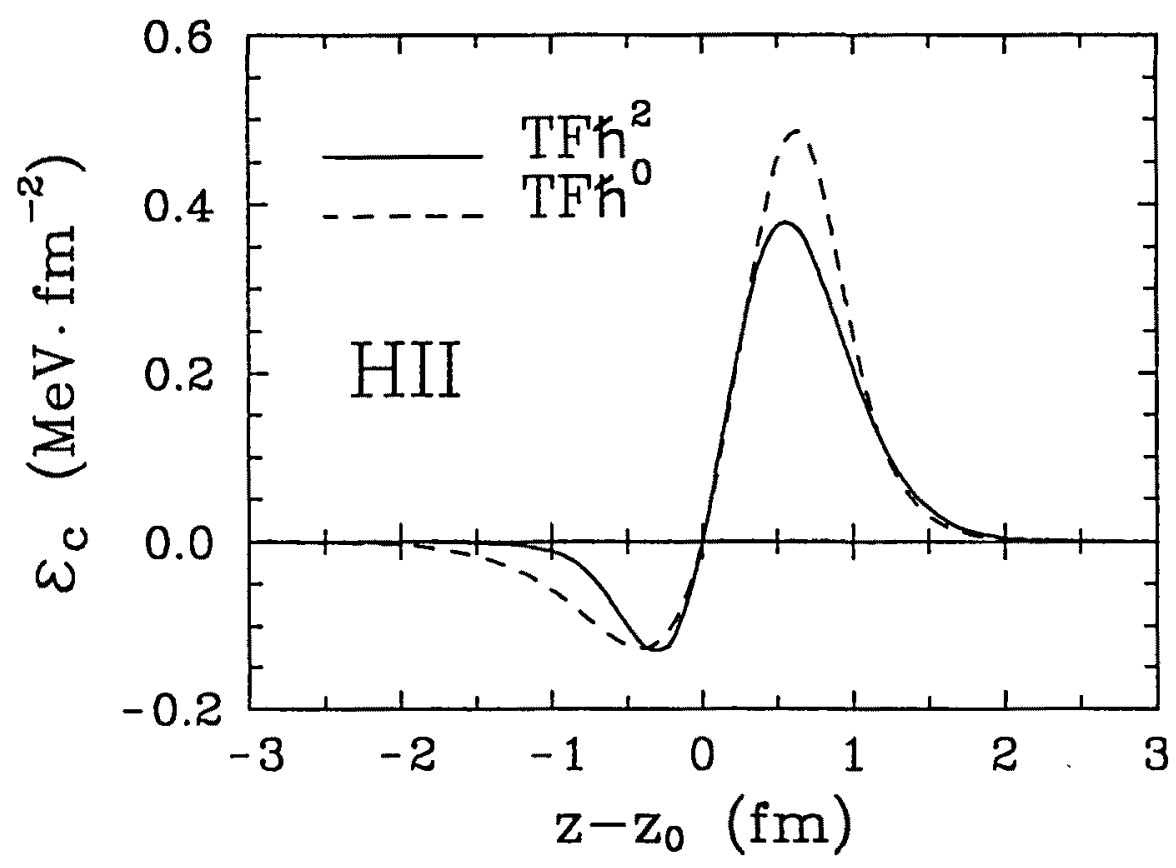

Figure 4.12. Curvature energy density $\varepsilon_{c}=\left(z-z_{0}\right)\left[\mathcal{E}(z)-a_{\mathrm{v}} \rho(z)\right]$ calculated with the interaction HII. 


\section{Chapter 5 .}

\section{Relativistic Extended}

\section{Thomas-Fermi Calculations of Finite Nuclei with Realistic Nucleon-Nucleon Interactions}

The UNDERSTANDING of the nuclear matter properties starting from a realistic NN interaction fitted to the NN scattering data has considerably been improved in recent years, as already mentioned, by the recognition of the importance of relativistic effects in treating nucleons inside a nucleus. The key point has been the observation that the dominant attractive and repulsive components of a realistic NN interaction exhibit different properties under a Lorentz transformation.

Within the OBE model, the strong attractive component of the NN interaction is described in terms of a scalar $\sigma$-meson exchange, while the repulsive component is dominantly due to the exchange of a vector $\omega$ meson. This structure of the NN interaction implies that the self-energy of the nucleons in nuclear matter, calculated from this interaction, contains a strong attractive scalar component and a strong repulsive component, which, in the rest frame of nuclear matter, transforms like the time-like component of a Lorentz-vector. Inserting this self-energy into the Dirac equation for 
the nucleons inside the nuclear medium, one obtains solutions for which the small component of the Dirac spinor is drastically enhanced as compared to the Dirac spinors for the free nucleons. This enhancement of the small component of the Dirac spinor for a nucleon at finite densities can be described in terms of an effective nucleon mass $m^{*}$ which is smaller than its bare mass. As a consequence of this change of the Dirac spinors for the nucleons as a function of the nuclear density, the matrix elements for the NN interaction and the kinetic energy are modified in the nuclear medium. These modifications are very important to describe the saturation properties of nuclear matter within a microscopic many-body calculation starting from a realistic NN interaction [An83, Br84, Ce86, Ma85, Ha87, Br90].

Such Dirac-Brueckner-Hartree-Fock (DBHF) calculations have also been carried out for the finite nucleus ${ }^{16} \mathrm{O}$ [Mü88, Mü90], using the Bonn potential [Br84, Ma85, $\mathrm{Br} 90]$ as the bare NN interaction. Also in this case the relativistic features just discussed lead to better results than the corresponding BHF ones [Sc91]. For finite nuclei, however, the relativistic effects considered in the DBHF approach are not sufficient to yield a complete agreement of the calculated binding energy and radii with the experimental data. Besides these attempts to solve the DBHF equations directly for finite nuclei, there exist also calculations on ground-state properties of finite nuclei assuming a LDA for the Dirac-Brueckner self-energies [Ma91].

Heavy ion (HI) scattering and large nuclear deformations are among the low and intermediate energy nuclear aspects less explored using RMF methods. We have seen in Chapter 4 that calculations of axially deformed nuclei have been carried out in relativistic mean field models, and the only calculations of $\mathrm{HI}$ optical potentials on the basis of the microscopic Dirac-Brueckner formalism have been carried out by the Tübingen group [Oh88].

In this chapter we want to employ our RETF method together with the DiracBrueckner results on nuclear matter of Ohtsuka et al. [Oh88] to evaluate the fission barriers of some selected nuclei and the complex optical potential for some HI systems at intermediate incident energies. For this purpose we shall use a LDA to build an energy density functional. The potential part comes from a microscopic calculation using a modern version of the realistic Bonn NN interaction, which incorporates the effects of Brueckner correlations and Dirac effects. The kinetic part is the RETF kinetic energy density constructed in Subsection 2.4.2. With respect to the results reported in [Oh88], the main improvement is that the use of the present energy density 
functional will allow us to obtain fully self-consistent nuclear densities, instead of using parameterized densities as in that reference. With respect to the previous work on fission barriers by Garcias et al. [Ga90b], the use here of a RMF method represents a sizable improvement, since in that reference we were using a non-relativistic NN interaction that was missing the nuclear matter saturation point, as all other nonrelativistic G-matrix calculations do [Sp72, Ma89, Sc91].

The chapter is organized as follows. In the first section we summarize the way the DBHF potential energy density has been obtained, construct the complete energy density functional and adjust its few free parameters, showing the results for finite nuclei. Next, we calculate the fission barriers for some selected nuclei, with the emphasis in the angular momentum dependence of these barriers. Finally, the HI potentials are obtained and some illustrative elastic HI scattering cross sections are calculated.

\subsection{The Energy Density Functional}

\subsubsection{The Dirac-Brueckner-Hartree-Fock potential energy density}

The nucleon Dirac spinor $\tilde{u}(k, \rho)$ for a nucleon of momentum $k$ in nuclear matter of density $\rho$ is determined by solving a Dirac equation

$$
\left[\gamma^{\mu} k_{\mu}-m-\Sigma(k, \rho)\right] \tilde{u}(k, \rho)=0
$$

where $m$ is the free nucleon mass, $\gamma^{\mu}$ are the Dirac matrices and $\Sigma(k, \rho)$ is the selfenergy operator of the nucleon. For symmetric nuclear matter this self-energy contains a large scalar component and a term, which, in the rest frame of nuclear matter, transforms like the time-like component of a Lorentz-vector:

$$
\Sigma(k, \rho)=A(k, \rho)+\gamma_{0} B(k, \rho) .
$$

In a simple relativistic mean field or Hartree approximation to the field theoretical model for the meson-nucleon many-body system, the coefficients $A(k, \rho)$ and $B(k, \rho)$ are independent of the momentum $k$ and are directly related to the attractive scalar and repulsive vector meson exchange contributions, respectively. Using a self-energy of the form given in Eq. (5.2), the solution of Eq. (5.1) for a positive energy nucleon is 
explicitly given by

$$
\tilde{u}(k, \rho)=\left(\frac{\varepsilon+m^{*}}{2 \varepsilon}\right)^{1 / 2}\left(\begin{array}{c}
1 \\
\frac{\sigma \cdot k}{\varepsilon+m^{*}}
\end{array}\right) \chi_{\sigma}
$$

where $m^{*}=m+A(k, \rho)$ is the nucleon effective mass and, again, $\varepsilon=\left(k^{2}+m^{* 2}\right)^{1 / 2}$. $\chi_{\sigma}$ is a Pauli spinor and the normalization is

$$
\tilde{u}^{\dagger}(k, \rho) \tilde{u}(k, \rho)=1
$$

In the Dirac-Brueckner approach, $\Sigma(k, \rho)$ is defined in terms of the $G$-matrix $\tilde{G}$ as

$$
\langle k|\Sigma| k\rangle=\sum_{\text {spin, isospin }} \int_{F} \frac{d k^{\prime}}{(2 \pi)^{3}}\left\langle k k^{\prime}\left|\tilde{G}\left(W=\epsilon_{k}+\epsilon_{k^{\prime}}\right)\right| k k^{\prime}\right\rangle .
$$

The integration is carried out over the Fermi sphere for nuclear matter of the density under consideration. The $G$-matrix $\tilde{G}$ is the solution of the relativistic Bethe-Goldstone equation for nuclear matter at rest. It is worth mentioning that, in addition to the selfconsistent treatment of the starting energy $W$ which is required in the non-relativistic BHF approach, the DBHF approach furthermore needs to treat the Dirac spinors selfconsistently. The spinors (5.3) resulting from Eq. (5.1) have to be used in evaluating the matrix elements of the NN potential in the nuclear medium. We have taken as the bare NN interaction the Bonn potential of [Br84, Ma85], which was already used by Ohtsuka et al. in [Oh88]. Employing Eq. (5.2) for $\Sigma(k, \rho)$, one finds that $A(k, \rho)$ and $B(k, \rho)$ are independent on the momentum $k$ to a very good approximation [El90]. The $k$-independent functions $A(\rho)$ and $B(\rho)$ are obtained by a fit to the numerical values for the self-energy of Eq. (5.5) as follows [Br84]:

$$
\langle k|\Sigma| k\rangle \approx A(\rho)+B(\rho)-\left(1-\frac{m}{m^{*}}\right) \frac{k^{2}}{2 m^{*}} .
$$

Consequently, the spinors of eq (5.3) can be parameterized in terms of a nucleon effective mass $m^{*}$ which depends on $\rho$ but not on $k$.

The potential energy density is readily obtained from the self-energy as

$$
\Pi(\rho)=\frac{4}{2} \int_{\mathrm{F}} \frac{d k}{(2 \pi)^{3}}\langle k|\Sigma(k, \rho)| k\rangle
$$


the factor 4 being due to spin-isospin degeneracy. In the relativistic treatment of nuclear matter, the kinetic energy density is given by

$$
\begin{aligned}
\tau(\rho) & =4 \int_{\mathrm{F}} \frac{d \boldsymbol{k}}{(2 \pi)^{3}} \tilde{u}^{\dagger}(k, \rho)(\alpha \cdot k+\beta m-m) \tilde{u}(k, \rho) \\
& =4 \int_{\mathrm{F}} \frac{d \boldsymbol{k}}{(2 \pi)^{3}}\left(\frac{k^{2}+m m^{*}}{\varepsilon}-m\right) .
\end{aligned}
$$

The relativistic approach makes the kinetic energy of nuclear matter less repulsive than in the non-relativistic approach, while on the other hand the potential energy (5.7) becomes much less attractive. As a sum, the total energy becomes less attractive at high density and the empirical values of binding energy and density of the nuclear matter saturation point are obtained.

\subsubsection{The complete energy density functional and parameter fit}

For any application to finite nuclei, it is desirable to use an energy density which incorporates some features not present in the simple functionals (5.7) and (5.8). We write the total energy of a nucleus as

$$
E=\int d \boldsymbol{r} \mathcal{E}\left[\rho_{q}(\boldsymbol{r}), \tau_{q}(\boldsymbol{r})\right],
$$

where the energy density functional $\mathcal{E}$ is given by

$$
\begin{aligned}
\mathcal{E}\left(\rho_{q}, \tau_{q}\right)= & \sum_{q} \tau_{q}+\alpha \Pi(\rho) \\
& +C_{\text {sym }}\left(\rho_{\mathrm{n}}-\rho_{\mathrm{p}}\right)^{2}+\eta(\nabla \rho)^{2}+\mathcal{E}_{\text {Coul }}\left(\rho_{\mathrm{p}}\right) .
\end{aligned}
$$

The term $\Pi$ has been introduced in the previous subsection. The RETF kinetic energy density

$$
\tau_{q}\left(\rho_{q}, m^{*}\right)=\tau_{0, q}\left(\rho_{q}, m^{*}\right)+\tau_{2, q}\left(\rho_{q}, m^{*}\right)
$$

is obtained from Eq. (2.81). In the nuclear matter limit, the $\hbar^{0}$ term $\tau_{0, q}$ would correspond to (5.8). We note that in our approach the nucleon effective mass $m^{*}$ depends only on the total density $\rho=\rho_{\mathrm{n}}+\rho_{\mathrm{p}}$.

The last three terms in (5.10) are purely phenomenological. The first one is a potential symmetry energy term, the gradient term accounts for a part of the surface 
energy, and the Coulomb energy $\mathcal{E}_{\text {Coul }}\left(\rho_{\mathrm{p}}\right)$ contains a direct term and a exchange term in the standard Slater approximation. The constant $\alpha$ in front of the potential energy density $\Pi(\rho)$ will be used to improve on the finite nuclei results. Altogether, our functional contains three free parameters $\alpha, C_{s y m}$ and $\eta$. Despite the phenomenological character that these parameters give to the energy density (5.10), connection with the microscopic calculation is kept in the model inasmuch as it is the density-dependent Dirac spinor, microscopically derived in the DBHF approach, which determines the behaviour of $\Pi$ and $\tau_{q}$ (through $m^{*}$ ) as a function of the density.

The nuclear symmetry energy has been obtained in the DBHF approximation [Mü87] so that, in principle, this could be used to fix the $C_{\text {sym }}$ parameter, and putting $\alpha=1$ we would have been left with only one free parameter $\eta$. However, as the NN interaction used in [Mü87] is different from the one used by Ohtsuka et al. [Oh88], for which $m^{*}$ and $\Pi(\rho)$ are available for many values of the density $\rho$ and for two nuclear matters in relative motion, we have preferred to take $\Pi(\rho)$ from the latter reference and have let $C_{\text {sym }}$ as a free parameter. The functional (5.10), made of the $\hbar^{2}$-order kinetic part and a potential part which contains the minimal phenomenology to make the whole functional realistic, has to be considered as a single package, which constitutes our model.

The Euler-Lagrange equations corresponding to the functional $\mathcal{E}\left(\rho_{q}, \tau_{q}\right)$ are solved self-consistently using the imaginary time-step method method, once a value for the set of parameters $\left(\alpha, C_{\text {sym }}, \eta\right)$ has been chosen. We shall present the results obtained with three different functionals. The first one will be called TUO and corresponds to the parameter-free case: $\eta=C_{\text {sym }}=0$ and $\alpha=1$. This functional, being the closest one to a microscopic calculation, in general is not expected to yield good results when compared to experiment because it lacks some surface and symmetry energy. It is interesting, however, to know how far one can go without including any kind of phenomenology in the model. TU1 is the second functional; to fix its parameters we have adjusted the binding energy of ${ }^{40} \mathrm{Ca}$ and ${ }^{208} \mathrm{~Pb}$, and the semiclassical fission barrier $\left(B_{\mathrm{f}}\right)$ of ${ }^{240} \mathrm{Pu}$, which is $\sim 3.8 \mathrm{MeV}[\mathrm{Br} 72, \mathrm{Br} 85]$ (details about the fission barrier calculation are given in the next section). The parameters of the third functional, called TU2, have been adjusted to reproduce the binding energy of ${ }^{40} \mathrm{Ca}$ and ${ }^{208} \mathrm{~Pb}$, and the value of the surface energy of semi-infinite symmetric nuclear matter corresponding to the $\mathrm{SkM}^{*}$ force, $a_{\mathrm{s}}=17.22 \mathrm{MeV}[\mathrm{Br} 85]$. 
It is very simple to obtain the surface tension $\sigma$ of semi-infinite symmetric nuclear matter for functionals like that of Eq. (5.10). Neglecting the Coulomb energy and taking $\rho_{\mathrm{n}}=\rho_{\mathrm{p}}=\rho / 2$, Eq. (5.10) can be written as

$$
\mathcal{E}(\rho)=C(\rho)+[D(\rho)+\eta](\nabla \rho)^{2},
$$

where

$$
C(\rho)=\sum_{q} \tau_{0, q}\left(\rho_{q}=\rho / 2\right)+\alpha \Pi(\rho),
$$

and $D(\dot{\rho})$ is such that

$$
D(\rho)(\nabla \rho)^{2}=\sum_{q} \tau_{2, q}\left(\rho_{q}=\rho / 2\right) .
$$

For a system with a planar surface perpendicular to the $z$-axis, $\rho=\rho(z)$. Following the method outlined in [Tr86], after some straightforward manipulations, one gets

$$
\begin{aligned}
\sigma & =\int_{-\infty}^{+\infty} d z\left[\mathcal{E}(\rho)-\frac{E}{A} \rho\right] \\
& =2 \int_{0}^{\rho_{0}} d \rho\left[C(\rho)-\frac{E}{A} \rho\right]^{1 / 2}[D(\rho)+\eta]^{1 / 2},
\end{aligned}
$$

where $E / A$ is the energy per particle at the saturation density $\rho_{0}$. The surface energy is

$$
a_{\mathrm{s}}=4 \pi r_{0}^{2} \sigma,
$$

where $r_{0}=\left[3 /\left(4 \pi \rho_{0}\right)\right]^{1 / 3}$ is the nuclear matter radius.

Table 5.1 collects the nuclear matter characteristics of the three functionals, which are well within the commonly accepted values except for a rather small symmetry energy $a_{\text {sym }}$ in the TUO case and, less markedly, in the TU1 case. The symmetry energy of TU0 is so small because for this functional only the kinetic energy contributes to it (the potential energy is calculated for $\rho_{\mathrm{p}}=\rho_{\mathrm{n}}$ ). The (bulk) symmetry energy is obtained by expanding the energy per particle of asymmetric nuclear matter for small values of the relative neutron excess $\left(\rho_{\mathbf{n}}-\rho_{\mathrm{p}}\right) / \rho$. The result for the functional (5.10) is

$$
a_{\mathrm{sym}}=\frac{k_{\mathrm{F}}^{2}}{6 \varepsilon_{\mathrm{F}}}\left[1-\frac{m m^{*}}{\varepsilon_{\mathrm{F}}^{2}}\left(1-\frac{m^{*}}{m}\right)\right]+C_{\mathrm{sym}} \rho,
$$

to be evaluated at the saturation density $\rho=\rho_{0}$. The first term in the r.h.s. of Eq. (5.17) comes from $\tau(\rho)$, and the second term from the phenomenological potential symmetry energy. 
Table 5.1. Parameters $\left(\alpha, C_{\text {sym }}, \eta\right)$ of the TU0, TU1 and TU2 functionals, their nuclear matter properties (energy per particle $E / A$, particle density $\rho_{0}$, Fermi momentum $k_{\mathrm{F}}$, incompressibility $\kappa$, effective mass $m^{*} / m$ ), volume symmetry energy $a_{\text {sym }}$ at saturation, surface energy $a_{\mathrm{s}}$ of semi-infinite symmetric nuclear matter, and fission barrier $B_{\mathrm{f}}$ of ${ }^{240} \mathrm{Pu}$.

\begin{tabular}{lccc}
\hline & TU0 & TU1 & TU2 \\
\hline$\alpha$ & 1.0 & 1.024 & 1.036 \\
$C_{\text {sym }}\left(\mathrm{MeV} \mathrm{fm}^{3}\right)$ & 0.0 & 84.0 & 118.0 \\
$\eta(\mathrm{MeV} \mathrm{fm})^{5}$ & 0.0 & 8.20 & 15.25 \\
$E / A(\mathrm{MeV})$ & -15.17 & -15.90 & -16.27 \\
$\rho_{0}\left(\mathrm{fm}^{-3}\right)$ & 0.1780 & 0.1778 & 0.1777 \\
$k_{\mathrm{F}}\left(\mathrm{fm}^{-1}\right)$ & 1.381 & 1.381 & 1.380 \\
$\kappa(\mathrm{MeV})$ & 239.2 & 250.2 & 255.7 \\
$m^{*} / m$ & 0.616 & 0.616 & 0.616 \\
$a_{\text {sym }}(\mathrm{MeV})$ & 9.48 & 24.41 & 30.45 \\
$a_{\mathrm{s}}(\mathrm{MeV})$ & 13.97 & 15.83 & 17.22 \\
$B_{\mathrm{f}}(\mathrm{MeV})$ & 2.2 & 3.7 & 5.6 \\
\hline
\end{tabular}

Table 5.2 shows the binding energies and charge radii we have obtained for some nuclei with the functionals TU0, TU1 and TU2. The calculated total energies have been corrected for spurious effects of centre-of-mass motion by subtracting the kinetic energy per particle, as it is usually done in non-relativistic calculations. The charge radii have been obtained from the proton radii as $r_{c}=\left(r_{p}^{2}+0.8^{2}\right)^{1 / 2}(\mathrm{fm})$ using a form factor for the proton charge. One may see from this table that the agreement between our calculations and the experimental data is globally good, especially for the binding energies. Even the parameter-free functional TU0 yields acceptable values and it is worthwhile noting the very small change ( $\alpha \approx 1$, Table 5.1) we have introduced in the potential part of both the TU1 and TU2 functionals. We want to point out again that as the spin-orbit is a fully relativistic effect, our expression for the kinetic energy density functional $\tau_{q}$ is automatically incorporating the $\hbar^{2}$-order corrections of spinorbit origin to that energy. Also, effective mass corrections are taken into account in $\tau_{q}$ up to the same order. This is conceptually important in the relativistic formalism, 
Table 5.2. Binding energies $B$ (in $\mathrm{MeV}$ ) and charge radii $r_{c}$ (in fm) obtained with the TU0, TU1 and TU2 functionals in comparison with experimental data.

\begin{tabular}{|c|c|c|c|c|c|c|c|c|}
\hline & \multicolumn{2}{|c|}{ TU0 } & \multicolumn{2}{|c|}{ TU1 } & \multicolumn{2}{|c|}{ TU2 } & \multicolumn{2}{|c|}{$\operatorname{Exp}$} \\
\hline & $B$ & $r_{c}$ & $B$ & $r_{\mathrm{c}}$ & $B$ & $r_{\mathrm{c}}$ & $B$ & $r_{\mathrm{c}}$ \\
\hline${ }^{12} \mathrm{C}$ & 94.2 & 2.49 & 94.3 & 2.53 & 92.4 & 2.57 & 92.2 & 2.47 \\
\hline${ }^{16} \mathrm{O}$ & 128.3 & 2.66 & 129.4 & 2.69 & 127.5 & 2.73 & 127.6 & 2.73 \\
\hline${ }^{40} \mathrm{Ca}$ & 333.1 & 3.36 & 342.3 & 3.37 & 342.2 & 3.40 & 342.1 & 3.49 \\
\hline${ }^{48} \mathrm{Ca}$ & 420.5 & 3.48 & 422.5 & 3.46 & 419.9 & 3.47 & 416.0 & 3.48 \\
\hline${ }^{56} \mathrm{Ni}$ & 461.1 & 3.70 & 476.9 & 3.70 & 479.0 & 3.72 & 484.0 & 3.75 \\
\hline${ }^{90} \mathrm{Zr}$ & 759.4 & 4.22 & 779.7 & 4.18 & 783.8 & 4.19 & 783.9 & 4.27 \\
\hline${ }^{114} \mathrm{Sn}$ & 942.1 & 4.53 & 967.2 & 4.49 & 973.7 & 4.50 & 971.6 & 4.61 \\
\hline${ }^{118} \mathrm{Sn}$ & 988.3 & 4.57 & 1006.0 & 4.52 & 1010.0 & 4.53 & 1005.0 & 4.64 \\
\hline${ }^{140} \mathrm{Ce}$ & 1157.5 & 4.81 & 1172.1 & 4.76 & 1176.0 & 4.77 & 1172.7 & 4.88 \\
\hline${ }^{208} \mathrm{~Pb}$ & 1641.6 & 5.46 & 1636.6 & 5.39 & 1636.5 & 5.39 & 1636.5 & 5.50 \\
\hline
\end{tabular}

and may be of some practical importance because of the large variations of $m^{*} / m$, as this ratio goes from $\sim 0.6$ at saturation (Table 5.1) to $\sim 1.0$ at low densities.

The TUO functional gives good binding energies for the smaller systems ${ }^{12} \mathrm{C}$ and ${ }^{16} \mathrm{O}$, without being necessary to add surface energy nor, obviously, symmetry energy. Remember that, compared with relativistic Hartree results, relativistic semiclassical calculations up to order $\hbar^{2}$ yield some overbinding (Subsection 4.2.4). This is the reason why the binding energy of ${ }^{16} \mathrm{O}$ obtained with TUO in this RETF approximation is in better agreement with the experimental value than the fully microscopic DBHF result [Mü88, Mü90]. With increasing neutron excess, the agreement of TU0 with experiment is spoiled. As TU0 has a small surface tension, it underestimates the semiclassical fission barrier of ${ }^{240} \mathrm{Pu}$ (Table 5.1) and that of any other nucleus, consequently. The TU2 force, which has the higher surface energy, yields slightly better results than TU0 and TU1, at the price of overestimating the fission barrier of ${ }^{240} \mathrm{Pu}$. Thus, the only force which is able to fulfil at the same time the requirements of yielding a proper description of ground-state properties and acceptable fission barriers is TU1. 
The TU0 parametrization gives better charge radii than TU1 and TU2. This is understood in the following terms. The lack of symmetry energy of TU0 is unable to counterbalance the Coulomb repulsion among protons. As a consequence, the proton density spreads out at the price of yielding unrealistically compact neutron densities. For example, the neutron r.m.s. radius of ${ }^{208} \mathrm{~Pb}$ is $5.29 \mathrm{fm}$ for TU0 and $5.44 \mathrm{fm}$ for TU1 (a HF calculation with the $\mathrm{SkM}^{*}$ force would give $5.63 \mathrm{fm}$ [Ce90a]). In any case, the calculated charge radii are systematically smaller than the experimental ones. This might be due to the fact that also the saturation density of nuclear matter calculated in the DBHF approximation is slightly above the empirical value (see Table 5.1). Note, however, that the radii we have found with the present RETF method are in much better agreement with the experimental ones than when one uses a non-relativistic $G$-matrix calculation as the input to obtain $\Pi(\rho)[\mathrm{Ga} 90 \mathrm{~b}]$.

\subsection{Fission Barriers}

In this section we apply the TU1 functional to the semiclassical description of symmetric nuclear fission. We do not present here results obtained with TU0 and TU2 because these functionals are unable to give a correct quantitative description of fission barriers, as discussed above in the case of ${ }^{240} \mathrm{Pu}$ (Table 5.1). The basic ingredients of the method we use have been described in [Ga90b] and references therein (see also [Ga89b]). It is based on a two dimensional (2D), axially symmetric Thomas-Fermi model that incorporates rotational effects.

To describe the symmetric fission of a nucleus, we have parameterized the fission path by a single collective coordinate, namely, the quadrupole moment $Q$. To obtain the equilibrium density at given values $Q=Q_{0}$ of the deformation and $L$ of the angular momentum, we have minimized the total energy with the usual constraints on $Q$ and $L_{x}$ :

$$
E+\frac{1}{2} \lambda\left(\langle Q\rangle-Q_{0}\right)^{2}-\omega\left\langle L_{x}\right\rangle
$$

where $E$ is given by Eqs. (5.9) and (5.10), and the Lagrange multiplier $\omega$ is to be identified with the angular velocity around the rotation axis $x$. We have imposed axial symmetry around the $z$-axis, and have solved the Euler-Lagrange equations corresponding to (5.18) in cylindrical coordinates $(r, z)$ employing the ITS method [Da85a, $\mathrm{Da} 85 \mathrm{~b}, \mathrm{Ga} 89 \mathrm{~b}]$. We have checked that the agreement we obtain between $1 \mathrm{D}$ and 2D calculations of spherical nuclei is better than 1-2 MeV in the total energy for the nuclei 
we have studied.

Rotation has been considered in the rigid body approximation [Si86]. At a given deformation, the moment of inertia $I_{x}$, needed to compute the rigid angular velocity

$$
\omega=\frac{\left\langle L_{x}\right\rangle}{I_{x}}
$$

and the rotational energy

$$
E_{\text {rot }}=\frac{\left\langle L_{x}\right\rangle^{2}}{2 I_{x}}
$$

is obtained from the self-consistent variational density $\rho(r, z)$ :

$$
I_{x}=m \iint d r d z 2 \pi r\left[z^{2}+\frac{r^{2}}{2}\right] \rho(r, z)
$$

as well as the quadrupole moment:

$$
\langle Q\rangle=\iint d r d z 2 \pi r\left[2 z^{2}-r^{2}\right] \rho(r, z) .
$$

We have collected in Table 5.3 the saddle point characteristics (barrier height and quadrupole moment) and the $L_{\mathrm{c}}$-values. We compare the RETF results with the ones we have obtained using the $\mathrm{SkM}^{*}$ force $[\mathrm{Br} 85]$ in a $\hbar^{2}$-order non-relativistic ETF calculation, and with the corresponding Liquid Droplet values [Co74, My77]. It can be seen that all three methods yield comparable results.

Fig. 5.1 is a three-dimensional plot of the closest-to-saddle configurations that we have calculated with TU1 for six nuclei, ranging from the light ${ }^{52} \mathrm{Fe}$ to the heavy ${ }^{240} \mathrm{Pu}$, without rotation. Some equidensity lines in the $(x, z)$ plane, corresponding to these configurations, are shown in Fig. 5.2. These figures show the known fact that the saddle configuration of a heavy nucleus is rather compact, whereas it is quite elongated for a light nucleus. The equidensity lines are parallel to a good approximation except those corresponding to high densities in the neck region. Similar contour plots can be found in [My91].

The evolution of the fission barrier as a function of the angular momentum is displayed in Fig. 5.3 for the case of ${ }^{118} \mathrm{Sn}$, which has in our model a critical angular momentum $L_{c}=102 \hbar$. It is seen that the configuration of minimum energy is no longer spherical for high enough values of $L$. Finally, a more detailed information about the $L$-dependence of the fission barrier height is given in Fig. 5.4, which represents the stability diagram of the nuclei we have studied. 
Table 5.3. Fission barrier $B_{\mathrm{f}}$ (in $\mathrm{MeV}$ ), quadrupole moment of the saddle point $Q_{\mathrm{s}}$ (in barn) and critical angular momentum $L_{c}$ (in units of $\hbar$ ), obtained with the TU1 functional in the RETF approach, with the SkM* force [Br85] in a $\hbar^{2}$-order non-relativistic ETF calculation, and with the Liquid Droplet model [Co74, My77].

\begin{tabular}{ccccc}
\hline & & TU1 & $\mathrm{SkM}^{*}$ & LD \\
\hline${ }^{52} \mathrm{Fe}$ & $B_{\mathrm{f}}$ & 47.7 & 49.0 & 48.0 \\
& $Q_{\mathrm{s}}$ & 28 & 29 & \\
& $L_{\mathrm{c}}$ & 48 & 55 & 50 \\
${ }^{118} \mathrm{Sn}$ & $B_{\mathrm{f}}$ & 56.5 & 55.6 & 52.6 \\
& $Q_{\mathrm{s}}$ & 119 & 140 & \\
& $L_{\mathrm{c}}$ & 102 & 101 & 95 \\
${ }^{152} \mathrm{Dy}$ & $B_{\mathrm{f}}$ & 36.4 & 34.1 & 36.7 \\
& $Q_{\mathrm{s}}$ & 180 & 200 & \\
& $L_{\mathrm{c}}$ & 87 & 91 & 95 \\
${ }^{186} \mathrm{Os}$ & $B_{\mathrm{f}}$ & 25.2 & 20.8 & 22.5 \\
& $Q_{\mathrm{s}}$ & 235 & 270 & \\
& $L_{\mathrm{c}}$ & 81 & 75 & 90 \\
${ }^{207} \mathrm{Bi}$ & $B_{\mathrm{f}}$ & 13.8 & 9.2 & 12.2 \\
& $Q_{\mathrm{s}}$ & 183 & 210 & \\
& $L_{\mathrm{c}}$ & 74 & 73 & 85 \\
${ }^{240} \mathrm{Pu}$ & $B_{\mathrm{f}}$ & 3.7 & 3.9 & 3.0 \\
& $Q_{\mathrm{s}}$ & 91 & 120 & \\
& $L_{\mathrm{c}}$ & 58 & 50 & 75 \\
\hline
\end{tabular}




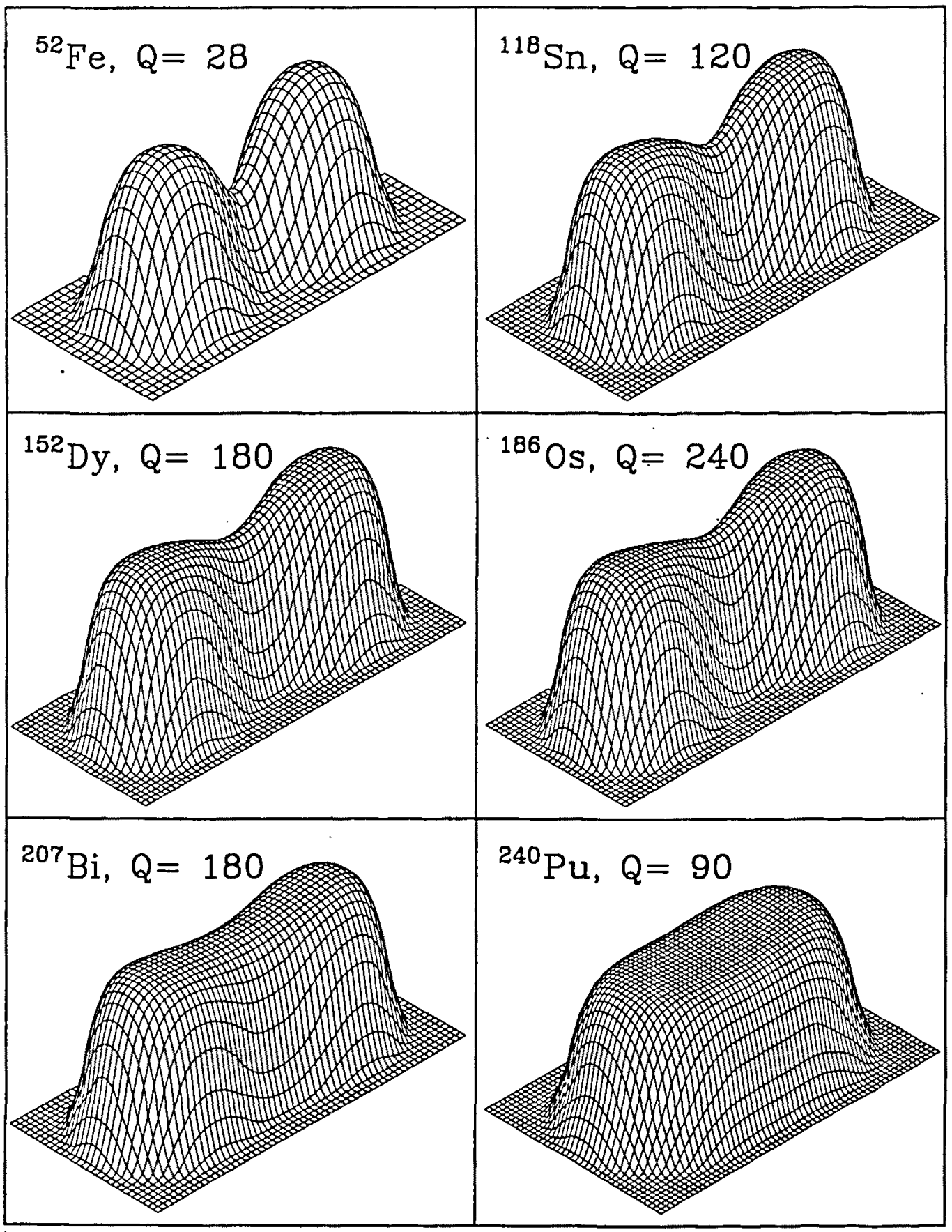

Figure 5.1. Three-dimensional isometric view of the density $\rho$, plotted versus the $(x, z)$ plane, for the closest-to-saddle configurations that we have calculated without rotation using the TU1 functional. The corresponding quadrupole moment $Q$ (in barns) is indicated for each nucleus. 


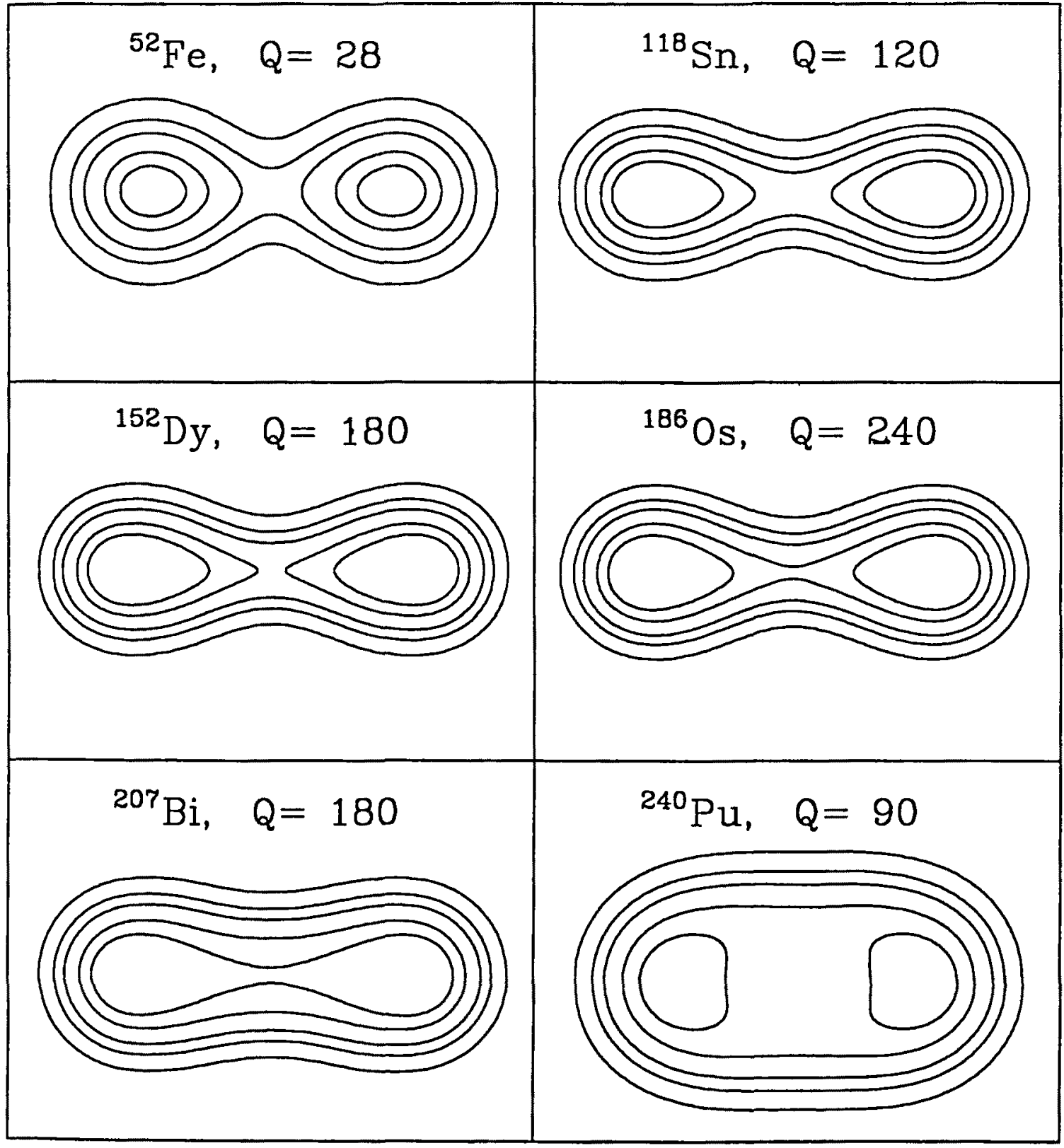

Figure 5.2. Equidensity lines in the $(x, z)$ plane corresponding to the configurations plotted in Fig. 5.1. From outside to inside, the lines represent contours of constant density $\rho=0.01$, $0.06,0.11,0.15$ and $0.165 \mathrm{fm}^{-3}$. 


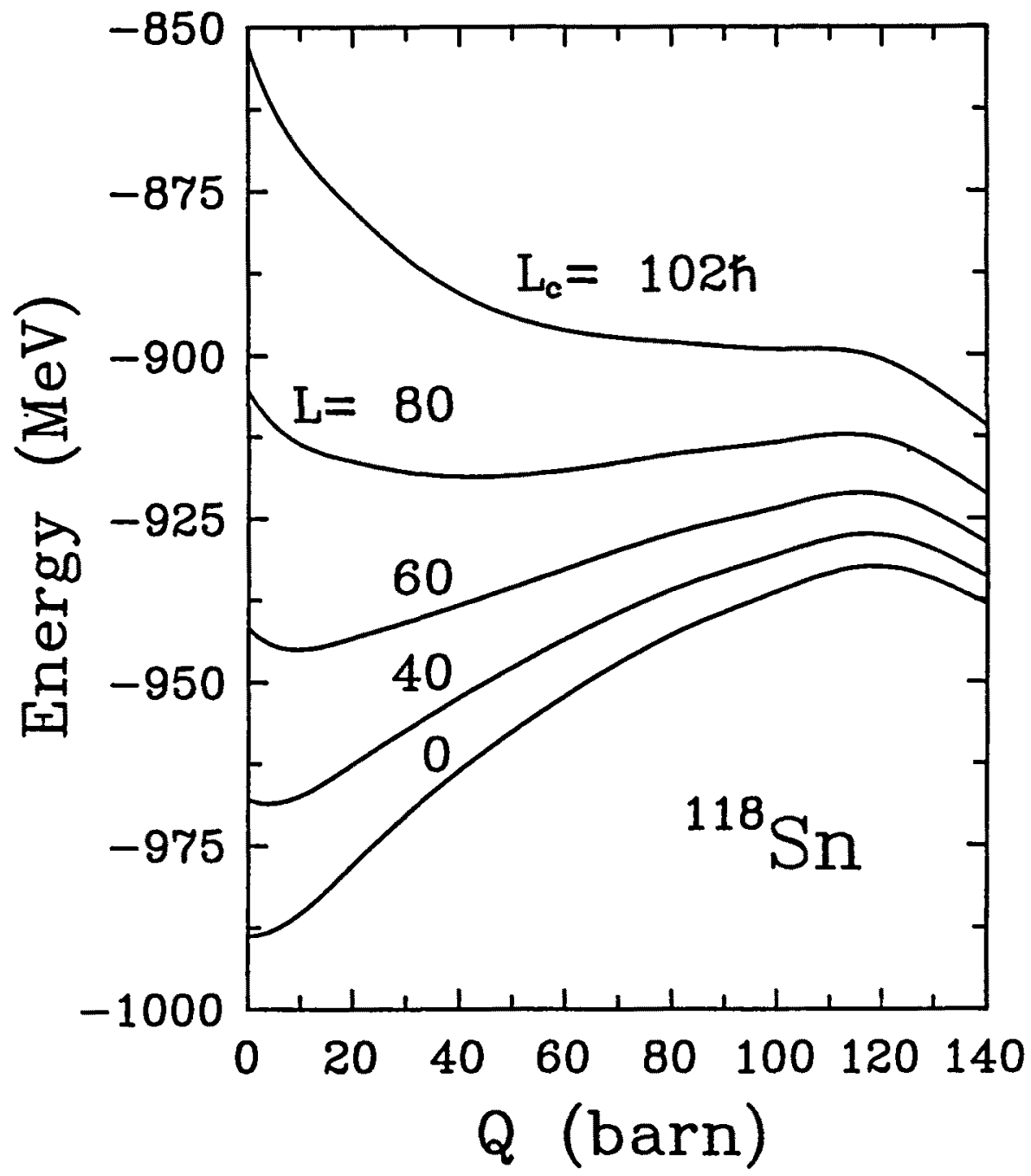

Figure 5.3. Angular momentum dependence of the fission barrier of ${ }^{118} \mathrm{Sn}$ as a function of the quadrupole moment $Q$ (with TU1). 


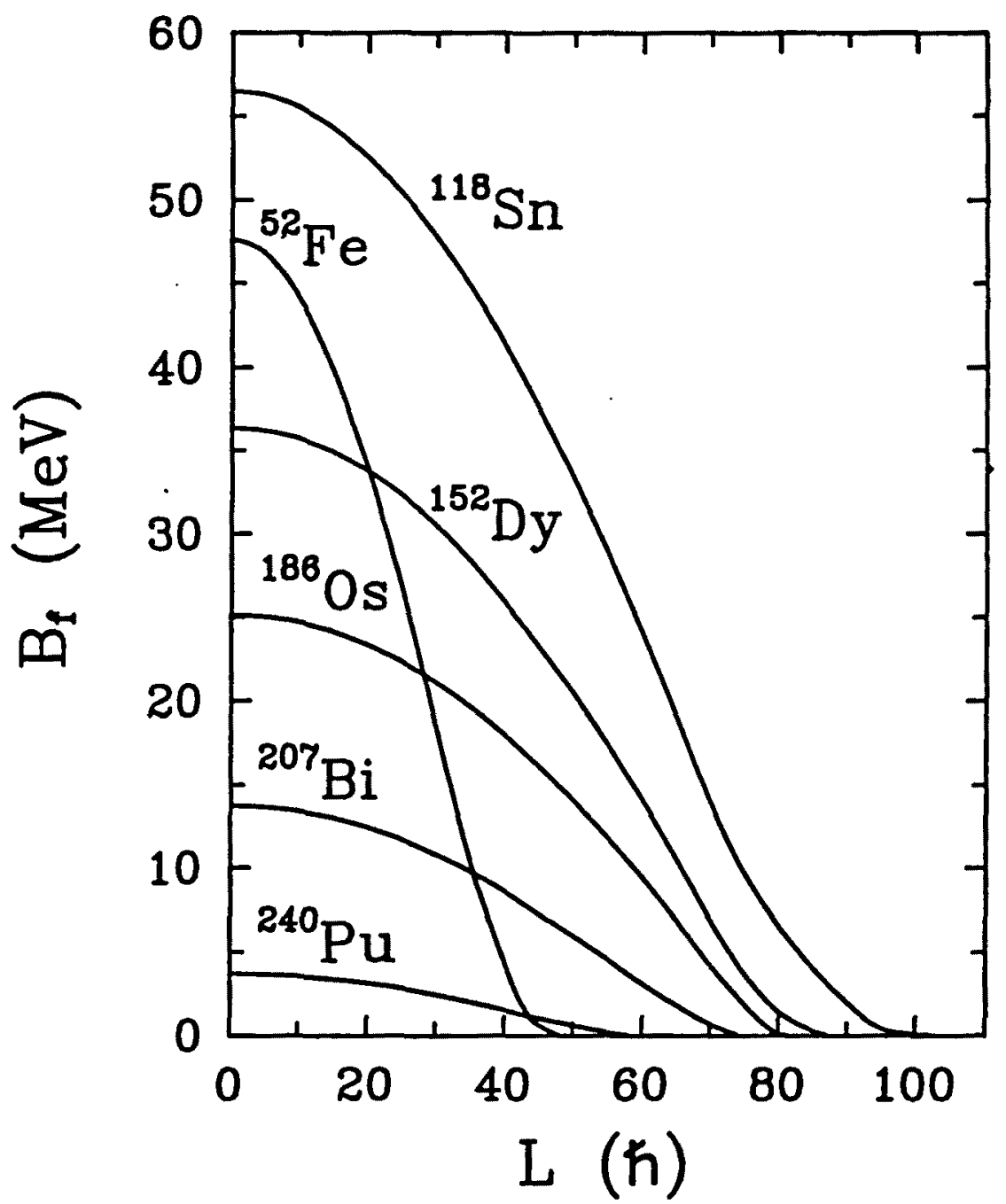

Figure 5.4. Stability diagram showing the evolution of the fission barrier height as a function of the angular momentum $L$ (with TU1). 


\subsection{Heavy Ion Optical Potentials at Intermediate Energies}

Using nuclear matter theory and the energy density formalism, the real and imaginary parts of the optical potential between two nuclei have been derived from realistic $\mathrm{NN}$ interactions by the Tübingen group in non-relativistic [Oh87] and relativistic approaches [Oh88]. These complex optical potentials have been successfully applied to describe the HI scattering data at intermediate energies. Therefore, it is of interest to see if the present formalism, which has been shown to give a satisfactory description of finite nuclei and fission barriers, can be used to improve on the relativistic calculation of the HI optical potential.

The optical potential between two nuclei at separation distance $R$ has been calculated as

$$
U\left(K_{\mathrm{r}}, R\right)=E\left(K_{\mathrm{r}}, R\right)-E\left(K_{\mathrm{r}}, \infty\right),
$$

using the sudden approximation for the density distribution of the composite system. In Eq. (5.23), $E\left(K_{\mathrm{r}}, R\right)$ is the total energy of the system formed by two nuclei whose centers are at distance $R$, approaching each other at such relative energy that the centers of the two Fermi spheres which represent the local densities of the target and the projectile are at a distance $K_{\mathrm{r}}$ (the relative momentum).

To obtain the potential energy density, it is assumed that the collision can be locally represented by a system of two nuclear matters colliding with relative momentum $K_{\mathrm{r}}$, and the total energy of the composite system is calculated using the nuclear densities obtained in Section 5.1 following the method indicated in [Oh88]. That is, instead of solving the relativistic Bethe-Goldstone equation for two colliding nuclear matters, the following steps are taken. First, the relativistic Bethe-Goldstone equation is solved for a single nuclear matter of density $\rho$ at rest, and the effective mass $m^{*}(\rho)$ and the matrix elements of the bare NN interaction between two nucleons in nuclear matter are determined in the same way as it has been done in [ $\mathrm{Br} 84, \mathrm{Ma} 85]$, and which has been already used to obtain the potential part $\Pi(\rho)$ employed in the previous sections. Then, these relativistic matrix elements are used to solve a non-relativistic Bethe-Goldstone equation for two nuclear matters with the total density of the combined system. By this procedure, proposed in [Oh88], the relativistic effects arising from the change of the Dirac spinors in the nuclear medium are included in this non-relativistic $G$-matrix since the matrix elements of the bare NN interaction are evaluated with the modified 
spinors, Eq. (5.3).

Especial attention has to be paid to the kinetic energy density in the case of two Fermi spheres in relative motion, and to the way to fix $K_{\mathrm{r}}$ for a given HI colliding system and incident energy. These matters are discussed in detail in [Oh88], and references therein.

One of the nicest features of the microscopic $G$-matrix approach to HI collisions is that in the case of two nuclear matters in relative motion, the potential energy becomes complex since two nucleons can be scattered into unoccupied states with conservation of their total momentum and energy. Thus one gets both the real and the imaginary part of the optical potential from the same interaction, and basically only the contributions of the mutual excitation of target and projectile are left out. As this contribution can be accounted for by standard coupled-channel techniques, the calculation of $\mathrm{HI}$ scattering cross sections becomes parameter-free.

Fig. 5.5 displays the real and imaginary parts of the optical potential for the ${ }^{12} \mathrm{C}+{ }^{12} \mathrm{C}$ system at 1016,1449 and $2400 \mathrm{MeV}$ in the laboratory frame, obtained with the TU1 functional (the ones yielded by TU0 and TU2 are very similar). A detailed comparison of the results of [Oh87] and [Oh88] reveals that the relativistic treatment affects the real part of the HI potentials obtained from two nuclear matters flowing through each other in a similar way it does for a single nuclear matter at rest. The change of the Dirac spinors reduces the attraction of the potential energy which is again counterbalanced by the reduction of the kinetic energy. The most striking feature of the relativistic approach to HI optical potentials is that the imaginary part is considerably enhanced at high densities. The change of the Dirac spinors reduces the attractive contributions of the $\sigma$-meson exchange but does not influence the repulsive $\omega$-meson exchange. Therefore, two nucleons at high densities feel a larger short-range repulsion, which leads to more scattering into unoccupied states, i.e., to a larger imaginary part. Note that this relativistic effect that comes from the potential energy is not compensated by any effect from the kinetic energy, as it happens for the real part.

We have used the calculated optical potentials to analyze some elastic HI scattering data. The elastic scattering cross sections have been obtained employing the PTOLEMY code [Ma78] in the optical model (OM) or coupled-channel (CC) analysis. As we have indicated above, our calculated HI potentials include the effect of $2 \mathrm{p}-2 \mathrm{~h}$ inelastic excitations, but not the excitation of $1 \mathrm{p}-1 \mathrm{~h}$ collective states which are absent in nuclear matter due to momentum conservation. They can be taken into account either 

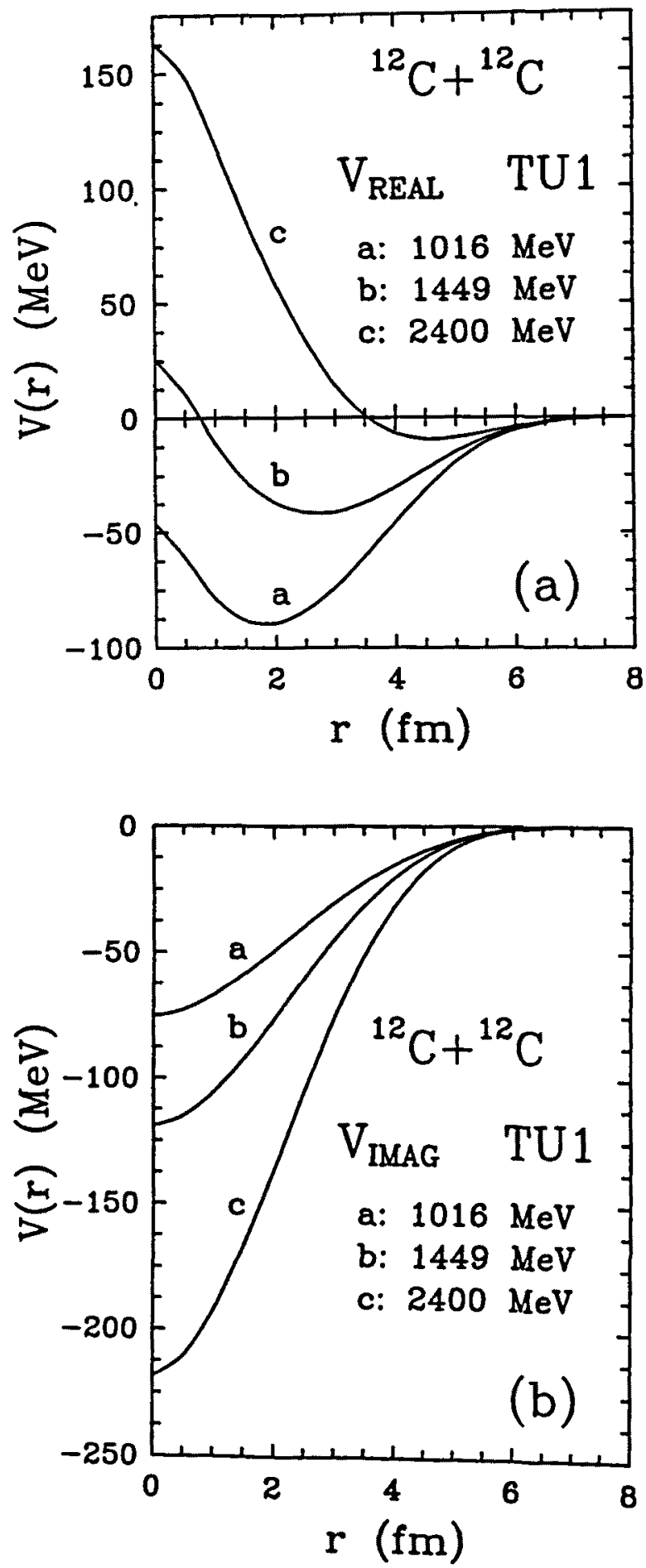

Figure 5.5. Real (a) and imaginary (b) part of the optical potential for ${ }^{12} \mathrm{C}+{ }^{12} \mathrm{C}$ at $E_{\mathrm{lab}}=1016,1449$ and $2400 \mathrm{MeV}$, obtained with the TU1 functional. 
as an additional effective potential [Kh81], or explicitly within the CC formalism. We have followed the latter approach, including the excitation of such collective states in both the target and the projectile.

We have studied the systems ${ }^{12} \mathrm{C}+{ }^{12} \mathrm{C},{ }^{16} \mathrm{O}+{ }^{12} \mathrm{C}$ and ${ }^{16} \mathrm{O}+{ }^{28} \mathrm{Si}$ at several incident energies. The collective states we have included into the $\mathrm{CC}$ analysis in the case of ${ }^{16} \mathrm{O}+{ }^{28} \mathrm{Si}$, are the lowest $2^{+}(6.92 \mathrm{MeV})$ state in ${ }^{16} \mathrm{O}$ and the $2^{+}(1.78 \mathrm{MeV})$ and $4^{+}(4.62 \mathrm{MeV})$ states in ${ }^{28} \mathrm{Si}$. For the ${ }^{12} \mathrm{C}+{ }^{12} \mathrm{C}$ system, we have included the first $2^{+}(4.44 \mathrm{MeV})$ and $3^{-}(9.64 \mathrm{MeV})$ states in ${ }^{12} \mathrm{C}$. For ${ }^{16} \mathrm{O}+{ }^{12} \mathrm{C}$ we have considered the first $2^{+}$and $3^{-}$in both nuclei, as in ${ }^{12} \mathrm{C}+{ }^{12} \mathrm{C}$. The coupling interaction is obtained adopting the vibrational or rotational model for the nuclear excited states. To get the nuclear transition potential $U_{l}$ for a given excited state, one needs to estimate the so-called nuclear deformation length $\beta_{\mathrm{N}} R_{\mathrm{N}}$. We have followed Blair's rule

$$
\beta_{\mathrm{N}} R_{\mathrm{N}}=\beta_{\mathrm{C}} R_{\mathrm{C}}
$$

to determine it. The deformation parameter $\beta_{\mathrm{C}}$ for the charge distribution is obtained from the electric transition probability $B(E l)$ using

$$
B(E l)=\left[\frac{3}{4 \pi} Z e R_{\mathrm{C}}^{l} \beta_{\mathrm{C}}(l)\right]^{2},
$$

with $R_{\mathrm{c}}=1.2 A^{1 / 3}(\mathrm{fm})$. Then, the transition potential follows from

$$
U_{l}(r)=\beta_{\mathrm{N}} R_{\mathrm{N}} \frac{d U_{0}(r)}{d r},
$$

where $U_{0}(r)$ is the complex optical potential previously determined. Thus, the CC calculations are essentially free of any adjustable parameter and constitute a good test of the reliability of the method.

Figs. 5.6 to 5.9 show the elastic scattering cross sections for the systems ${ }^{12} \mathrm{C}+{ }^{12} \mathrm{C}$ at $E_{\mathrm{lab}}=1016,1449$ and $2400 \mathrm{MeV},{ }^{16} \mathrm{O}+{ }^{12} \mathrm{C}$ at $E_{\mathrm{lab}}=1503 \mathrm{MeV}$ and ${ }^{16} \mathrm{O}+{ }^{28} \mathrm{Si}$ at $E_{\mathrm{lab}}=1503 \mathrm{MeV}$, respectively. The experimental data have been taken from [Bu84, Ho88b, Ro85, Ro87]. For the systems ${ }^{12} \mathrm{C}+{ }^{12} \mathrm{C}$ at $E_{\text {lab }}=1016$ and $1449 \mathrm{MeV}$ and ${ }^{16} \mathrm{O}+{ }^{28} \mathrm{Si}$ at $E_{\mathrm{lab}}=1503 \mathrm{MeV}$ we display both the $\mathrm{OM}$ and the $\mathrm{CC}$ results, calculated with the TU1 functional. In the case of the systems ${ }^{12} \mathrm{C}+{ }^{12} \mathrm{C}$ at $E_{\text {lab }}=2400 \mathrm{MeV}$ and ${ }^{16} \mathrm{O}+{ }^{12} \mathrm{C}$ at $E_{\text {lab }}=1503 \mathrm{MeV}$, we instead present only the $\mathrm{CC}$ analysis and compare the TU0, TU1 and TU2 results. The latter figures evidence that the three functionals yield comparable results for the cross sections of the systems under consideration. For 


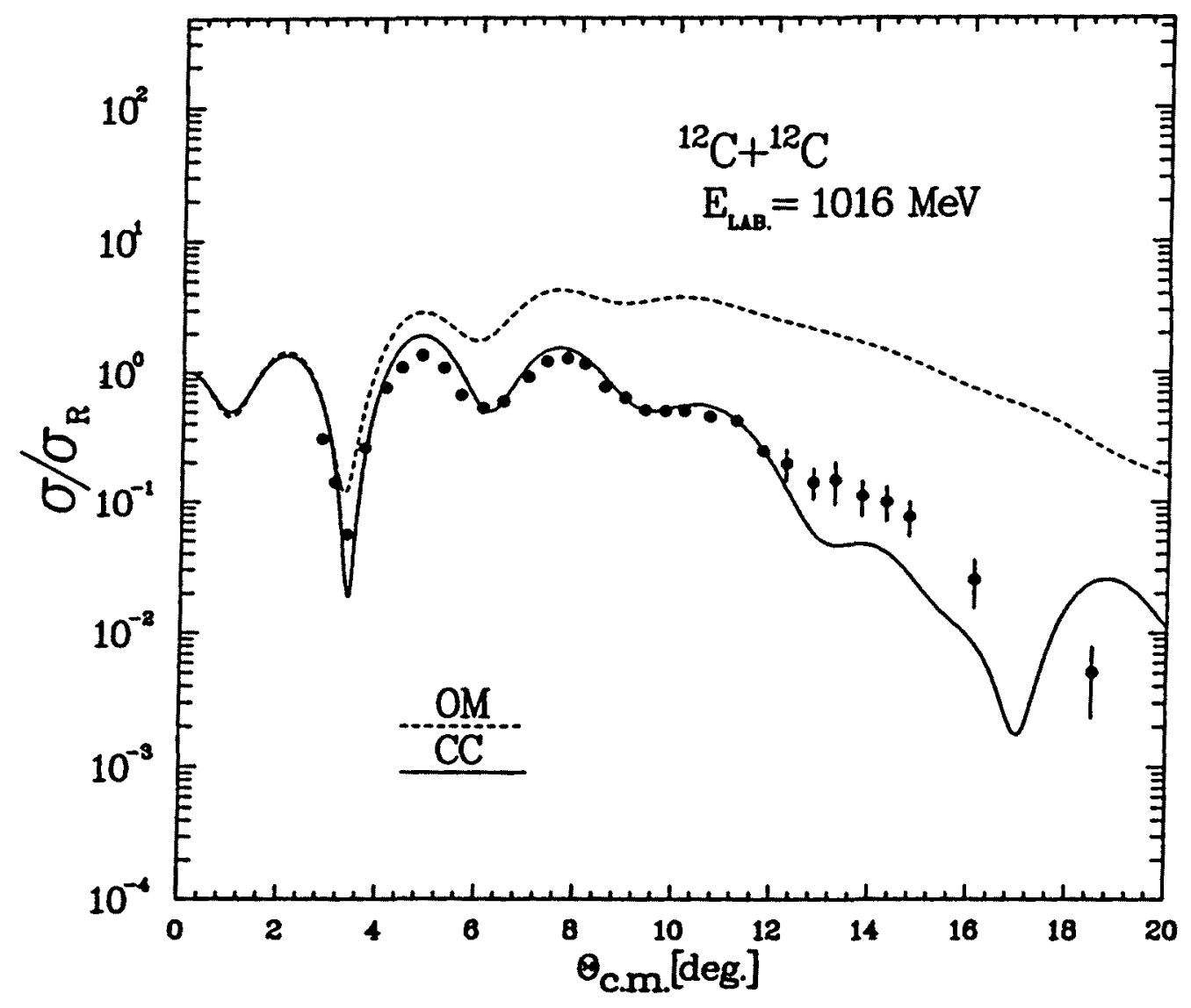

Figure 5.6. Elastic scattering cross section for ${ }^{12} \mathrm{C}+{ }^{12} \mathrm{C}$ at $E_{\mathrm{lab}}=1016 \mathrm{MeV}$ calculated with the TU1 functional. Dashed line, optical model (OM) analysis. Solid line, coupled channel (CC) calculation. The experimental data are from [Bu84].

this comparison one should be aware that the surface tension contents in TU1 and TU2 gives an attractive contribution to the real part of HI potential at the surface. This is counterbalanced by the global enhancement factor $\alpha$ in such a way that the real parts of TU0, TU1 and TU2 potentials are very similar at the surface region which is most sensitive to HI scattering data. Consequently, the calculated TU0, TU1 and TU2 cross sections for elastic HI scattering are also very similar.

The figures show that our potentials are reproducing the data quite reasonably in the CC calculations. The role of the coupling to the lowest collective excitations in the colliding nuclei is substantial at the energies considered here. In other OM analysis using phenomenological optical potentials this effect is hidden by the adjustable 


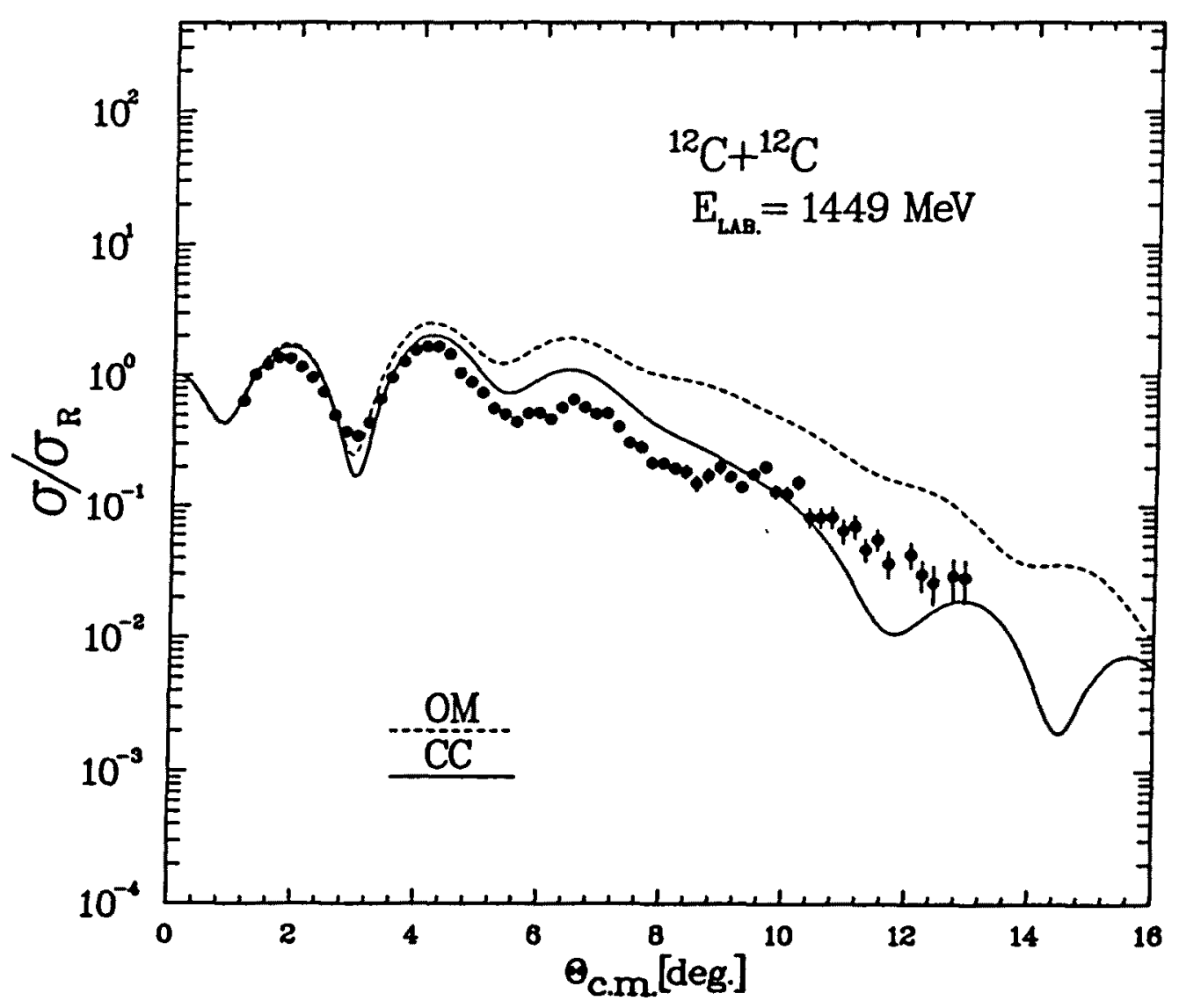

Figure 5.7. Same as Fig. 5.6 for $E_{\mathrm{lab}}=1449 \mathrm{MeV}$. The experimental data are from [Ho88b].

parameters of the potentials and, consequently, little information about their explicit contribution to the cross section can be extracted. Comparing the $\mathrm{OM}$ and $\mathrm{CC}$ cross sections of the ${ }^{12} \mathrm{C}+{ }^{12} \mathrm{C}$ reactions, we find that the effect of the $1 \mathrm{p}-1 \mathrm{~h}$ contributions to the optical potential decreases with increasing energy. This shows the diminishing contribution of the nuclear surface excitations to the elastic scattering as the energy increases. The remaining discrepancy with the data, basically the lack of absorption, is expected to be due to other inelastic processes which still are not taken into account by the CC calculation, like nucleon transfer between target and projectile, and especially alpha particle transfer [Di89]. 


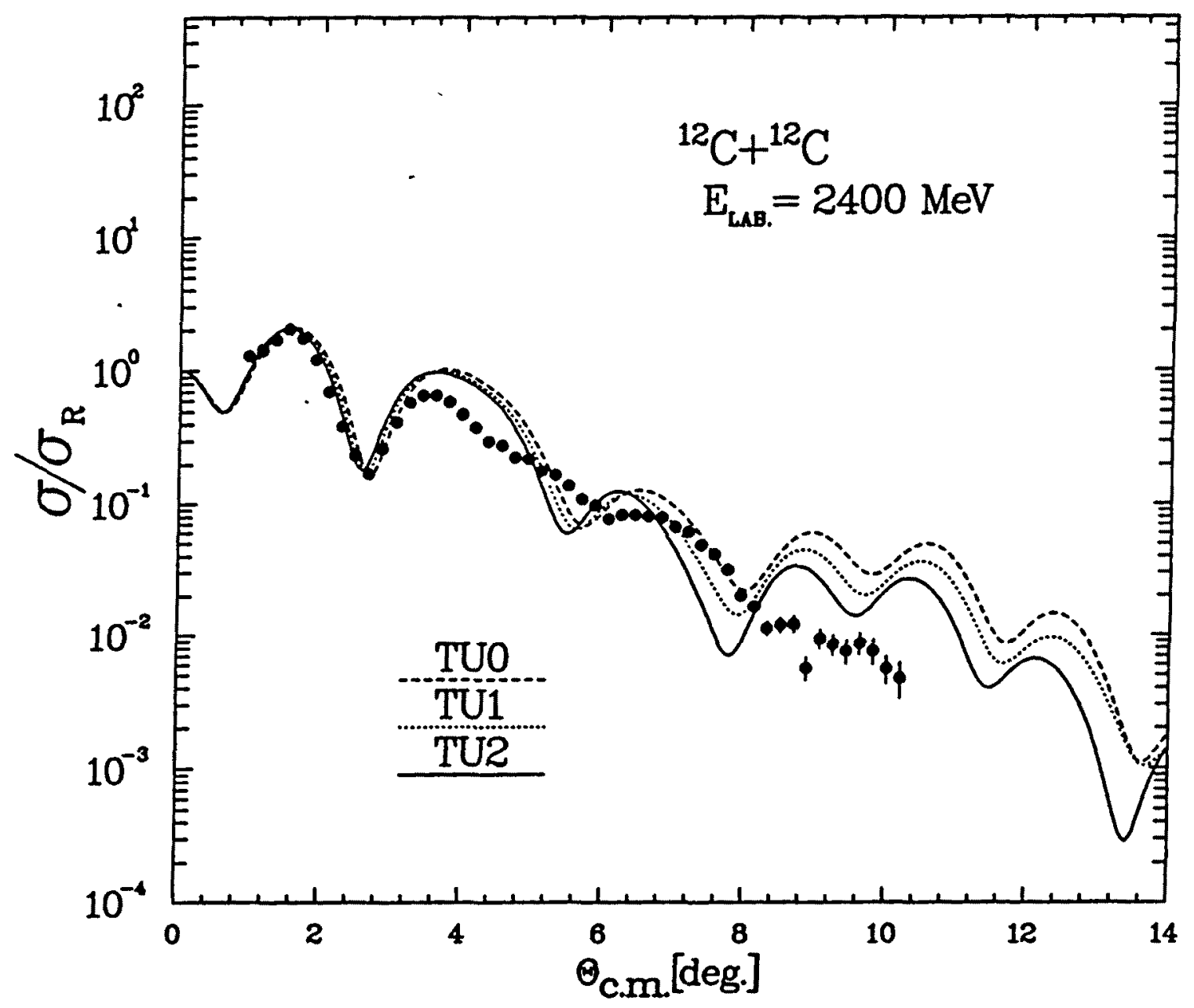

Figure 5.8. Elastic scattering cross section for ${ }^{12} \mathrm{C}+{ }^{12} \mathrm{C}$ at $E_{\mathrm{lab}}=2400 \mathrm{MeV}$ calculated in the CC analysis. Dashed line, using the TU0 functional; dotted line, TU1; and solid line, TU2. The experimental data are from [Ho88b]. 


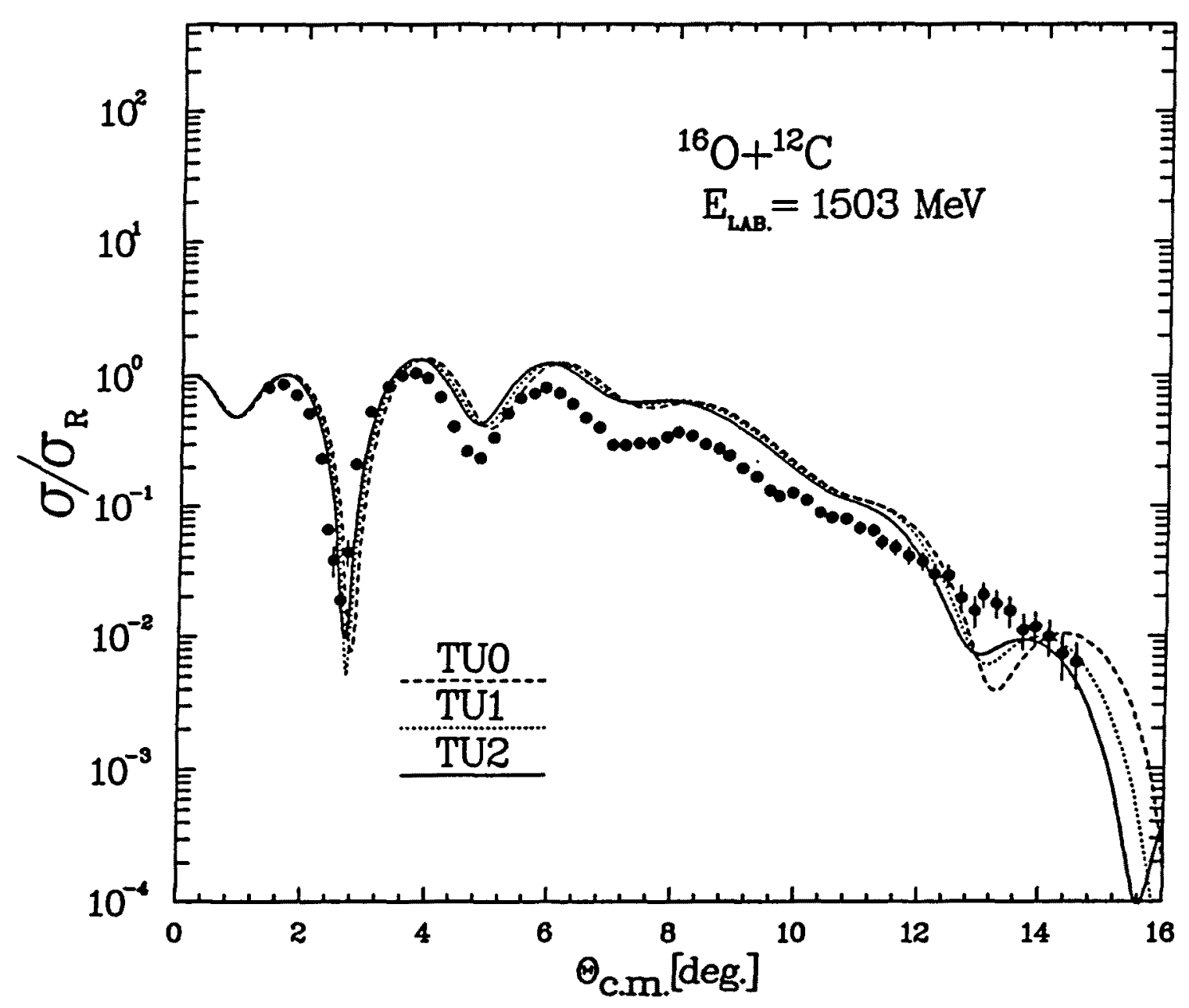

Figure 5.9. Same as Fig. 5.8 for the system ${ }^{16} \mathrm{O}+{ }^{12} \mathrm{C}$ at $E_{\mathrm{lab}}=1503 \mathrm{MeV}$. The experimental data are from [Ro85]. 


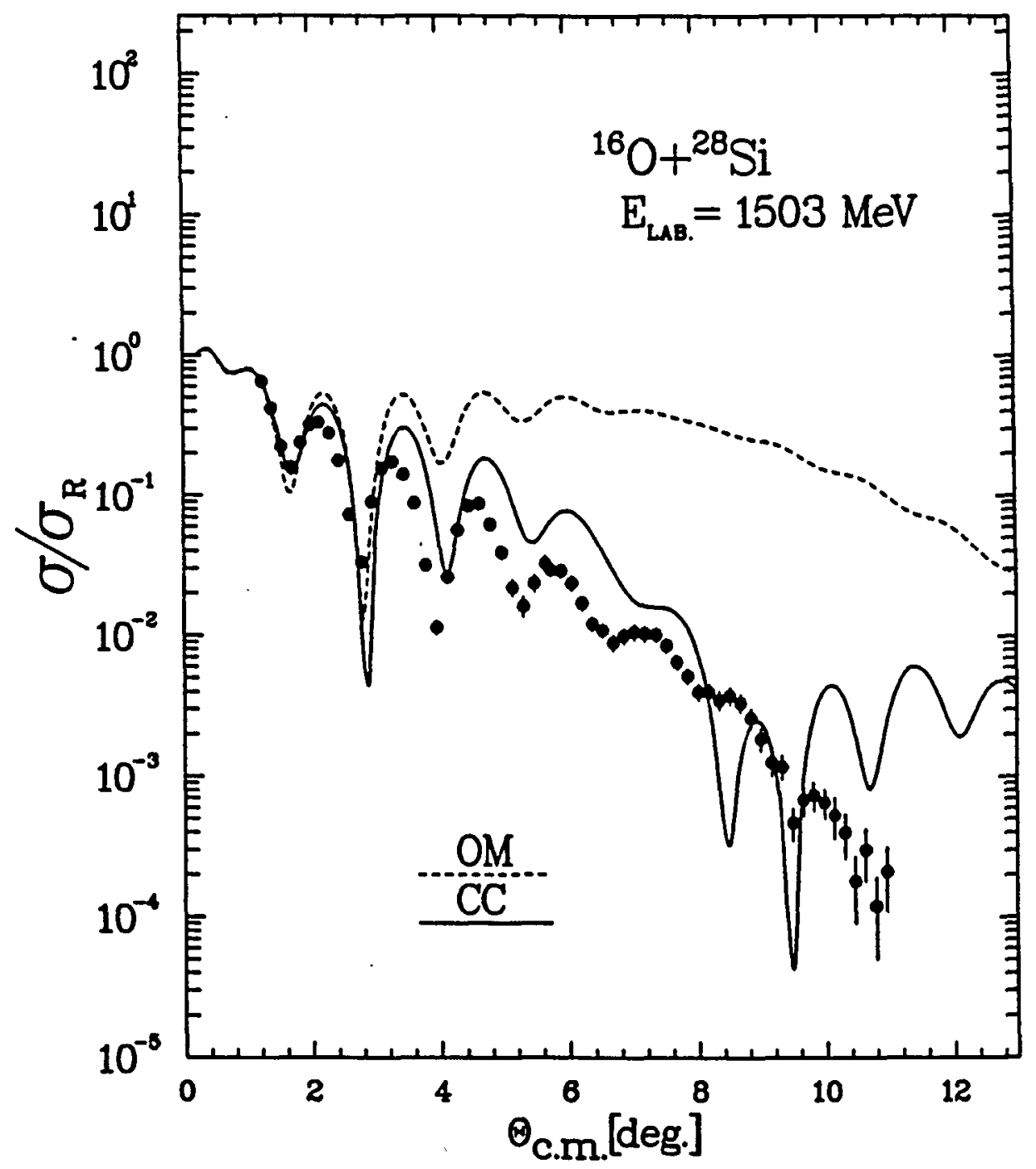

Figure 5.10. Same as Fig. 5.6 for the system ${ }^{16} \mathrm{O}+{ }^{28} \mathrm{Si}$ at $E_{\text {lab }}=1503 \mathrm{MeV}$. The experimental data are from [Ros7]. 


\section{Summary and Concluding Remarks}

THE PROBLEMS involving bulk properties of nuclei such as nucleon distributions and binding energies still are among some of the most pertinent topics of modern nuclear physics. They have been and are being attacked from many different angles. We know that the complete description of nuclear structure is contained in the quantal wave function of the nucleus. But we also know that it is practically impossible to determine theoretically all details of the behaviour of a many-body system of such complexity as a nucleus. Even in the situations where it is possible to solve for the complicated nuclear wave functions explicitly, and despite we are living an era where the amazing progress of computers allows to perform calculations which only a few years ago would have required prohibitively large computation times, it is still definitely desirable and necessary to develop simpler models than the quantal formulation. Ideally, such simplified models should contain all essential features of the nuclear problem and make the nuclear behaviour understandable in easy terms, so that a vivid picture of the nucleus could emerge from them. In the recent years, semiclassical methods have shown to be especially well suited for that purpose.

On the other hand, nuclear physics is nowadays being increasingly approached on the basis of a relativistic formalism. Microscopic DBHF calculations seem to be very promising. The theory of quantum hadrodynamics provides a consistent framework for a relativistic description of the nucleus. In this theory the degrees of freedom are the nucleons and the mesons, and the dynamics is specified by renormalizable, local, 
Lorentz covariant Lagrangian densities. The simple mean field approach permits the model to be solved analitically for nuclear matter. Calculations in quantal Hartree approximation give an accurate description of many of the features of nuclear systems. From the semiclassical viewpoint, solutions of the model relied on the lowest order Thomas-Fermi approximation. In this approximation, the particles at each point in space feel the potential as if it were locally equal to a constant. Thus, it is not very appropiate to handle many-body nuclear systems because of the short-range two-nucleon interactions. In particular, the nuclear surface properties cannot be described very well with the simple Thomas-Fermi theory.

We feel that all of these reasons encourage-and justify more than enough-the motivation and the interest for undertaking a systematic investigation on the relativistic nuclear many-body problem within a semiclassical framework which provides a consistent procedure to perform the expansion of physical quantities in powers of $\hbar$. Noticeably enough, such a theory was missing in nuclear physics.

The work presented in this thesis, therefore, constitutes a first step in this direction. We have addressed technical aspects of relativistic semiclassical theory, complemented with illustrative applications to finite nuclei and semi-infinite nuclear matter. We are well aware that a meaningful question is whether or not $\hbar$ corrections are important for the relativistic nuclear problem. It may seem that the phenomenological character of the relativistic nuclear models does not necessitate such complications. Let us remind, however, that the Skyrme forces used in the non-relativistic context are also based on phenomenological models; nevertheless, it was necessary to introduce $\hbar$ corrections in semiclassical calculations to accurately describe some quantities, e.g., the surface energy. The same consideration applies to the relativistic problem. Furthermore, the fact that in the latter case the nuclear potential is given by a difference of two large numbers makes the introduction of gradient corrections specially relevant.

We have presented a (new) recursive scheme to obtain the semiclassical $\hbar$ expansion of the propagator associated with a time-independent single-particle hamiltonian with matrix structure. In analogy to the non-relativistic case, the approach is based on the Wigner-Kirkwood expansion of the phase-space densities. We have focussed on the application of the method to a Dirac hamiltonian related to relativistic nuclear mean field theory, i.e., including a position-dependent effective mass and the time-like component of a four-vector field. Our $\hbar$ corrections just derive from spatial inhomogeneities, whereas time is always resummed to all orders. The method can also be used 
in other problems for which the hamiltonian has matrix components [Ta92].

Compared with the non-relativistic case, the procedure is considerably more complicated owing to the matrix structure of the hamiltonian. For this reason the $\hbar$ expansion was pushed to order $\hbar^{2}$ only. A detailed derivation has been given of the $\hbar^{2}$-order Wigner-Kirkwood expansion of the relativistic density matrix, as well as of the particle and energy densities. We have shown the idempotency of the semiclassical density matrix to second order in $\hbar$, which is a subtle proof since it involves the evaluation of products of distributions. This is a subject of general interest in the context of semiclassical theory. The Wigner-Kirkwood expressions, as they stand, are not suitable to be employed in a self-consistent problem. Therefore, we obtained the corresponding density functional results, where the energy densities are expressed as a functional of the local density. Complementarily, we have traced out the non-relativistic limit of the calculated expressions.

The accuracy of the Wigner-Kirkwood series was tested on a relativistic harmonic oscillator and perfect agreement with the Strutinsky averaged observables was found even in the highly relativistic regime. The density functional version was shown to be slightly less accurate, a feature already known in the non-relativistic case. It turns out that the semiclassical expressions represent the different quantities (only) on the average, that is, quantum fluctuations are averaged out. This model study shows that, for positive energy states, the semiclassical expansions we have derived contain all the relativistic ingredients, the difference with quantal results being due mainly to shell effects.

The method we have introduced to perform the semiclassical expansion of the relativistic nuclear problem is certainly not unique. In the course of our work, other authors (the München group of Weigel [We91, Ei91a, Ei91b] and the Frankfurt group of Dreizler [Sp92, Mü92]) addressed extensively the same problem with Green's function techniques. We have attempted to give in short a perspective of the relationship between these approaches and our. These authors have extended our investigation in several respects: Fock terms have been included, the spatial components of the fourvector potential and vacuum contributions have been considered, and the theory has been formulated at finite temperature. Nonetheless, to our knowledge, the numerical applications we have investigated remain the only ones carried out with a RETF method to date.

We have performed Extended Thomas-Fermi calculations for relativistic non-linear 
$\sigma-\omega$ models using two kinds of Lagrangians which differ in the form of the scalar coupling for the isoscalar sigma meson. Comparing $\mathrm{TF} \hbar^{0}$ with Hartree results, we have found that the $\mathrm{TF} \hbar^{0}$ approximation yields some underbinding when the effective mass of the model is small, and some overbinding when $m^{*}$ is large. For a value around $m^{*} / m=0.65$, both TF $\hbar^{0}$ and Hartree would roughly yield the same binding energy.

When the $\hbar^{2}$-order gradient corrections are taken into account, we have found a numerical instability in the solution of the semiclassical Klein-Gordon equation obeyed by the scalar field in the case of parametrizations which have $m^{*} / m \leq 0.60$. Whether this instability is due to the particular nature of the $\sigma-\omega$ model or to a failure of our semiclassical expansion of order $\hbar^{2}$, is an open question that can only be answered when this expansion be applied to other relativistic models.

Second order corrections in $\hbar$ to the $\mathrm{TF} \hbar^{0}$ approximation improve the agreement with Hartree solutions in a sensitive way, always yielding more bound nuclei than within the Hartree approach. The sign of the $\hbar^{2}$ corrections depends on $m^{*}$, and they are found to vanish around $m^{*} / m=0.75$ for the models of the type considered here. In several respects, the semiclassical relativistic phenomenology quite resembles the one met in the non-relativistic regime using Skyrme forces, in spite of the different origin of $m^{*}$ in both situations. In the interior of the nucleus the Hartree densities are well represented on the average by $\mathrm{TF} \hbar^{0}$ and $\mathrm{TF} \hbar^{2}$. But in the surface and the outer region the $\mathrm{TF} \hbar^{2}$ densities come appreciably closer than $\mathrm{TF} \hbar^{0}$ to the Hartree results, showing an exponential drop off. Using the $\mathrm{TF} \hbar^{2}$ semiclassical mean field as a starting point, perturbative quantal solutions $\left(\mathrm{H}^{*}\right)$ have been found which are in good agreement with the exact Hartree results.

One of the most appealing merits of a semiclassical formulation is the fact that certain observables are only accessible in a semiclassical framework. This is for instance the case of the curvature energy because quantally the content in curvature of the wave function is unknown. Therefore, we have calculated liquid drop model coefficients for some parameter sets of the $\sigma-\omega$ model, and have found reasonable values for the surface thickness and for the surface and curvature energies.

In this work we also wanted to study the effects of the density-dependent Dirac spinor for the nucleons, as it is determined microscopically in the DBHF approach, on various properties of the structure and scattering of finite nuclei. For that purpose, we have constructed a relativistic energy density functional that includes our kinetic energy density functional of order $\hbar^{2}$ and a volume part in the potential energy arising 
from a DBHF calculation of nuclear matter. This volume term is supplemented by some conventional correction terms and the few free parameters are suitably adjusted. It turns out that the radii of nuclei calculated with the present approach are in a better agreement with the experimental value than those obtained in similar studies using a potential energy derived from a non-relativistic G-matrix. This demonstrates that the Dirac effects improve the calculation of ground-state properties of finite nuclei also in our RETF approximation.

We want to stress, however, that this study of ground-state properties was not the main goal of our investigation. For such studies direct microscopic calculations are possible [Mü88, Mü90] and, as we have seen, other more sophisticated phenomenological relativistic theories are working well ( $\sigma-\omega$ models). The capabilities of our RETF functional are actually appraised in situations in which a full microscopic relativistic calculation, or even a phenomenological one, cannot be easily made, like nuclear fission of rotating nuclei and HI scattering. In these situations, the method constitutes a reliable tool. For the nuclear fission barriers, the present calculations are the first ones carried out with a relativistic model. We have shown that the model yields results comparable to the non-relativistic ones with the conceptual advantage of being relativistic. For the calculations of $\mathrm{HI}$ elastic scattering cross sections, we have been able to improve the results that the Tübingen group had previously obtained [Oh88] due to achieving a better description of the nuclear densities.

Clearly, there are still several stimulating topics for continuation and future research in the field:

- Study of the level density parameter with the relativistic semiclassical formalism.

- Analysis of giant resonances in the framework of the sum-rule approach.

- Semiclassical calculation of fission barriers in the $\sigma-\omega$ model following the lines of Section 5.2 .

- Some authors have pointed out that relativistic effects may play a significant role in the nuclear surface and may need to be taken into account in the calculation of the curvature energy coefficient [St88]. The results for semi-infinite nuclear matter that we have given here should be considered as preliminary only. A systematic study of the surface and curvature properties of nuclear matter using the RETF method remains to be carried out. The dependence of such properties 
upon the effective mass and the incompressibility of different parametrizations of the $\sigma-\omega$ model can be analyzed with the method outlined in Section 4.3, and the calculations can be extended to the derivative scalar coupling.

- A $\hbar^{2}$-order density functional description of QHD at finite temperature has been given in [Mü92]. As in the non-relativistic case, one might attempt to investigate numerical applications in thermal nuclear systems, where semiclassical methods are specially accurate because shell effects are washed out by temperature and the $\hbar$ expansions converge faster.

- A by far more ambitious project would be to explore, in the semiclassical context, the importance of corrections to the mean field approximation. In order to improve the mean field results one could include, for instance, exchange contributions, the effects of vacuum fluctuations or allow for currents in the description of axially deformed nuclei.

To conclude, we would like to stress that we have been able to find well-behaved solutions of the semiclassical variational equations, whose existence was not evident a priori. Since the semiclassical expansion of the energy density functional has likely not converged if one stops the expansion at the $\hbar^{2}$-order terms, this renders the search for higher order $\hbar$ corrections a worthy and non-merely academic task. 


\section{Appendix A}

THE EXPRESSION of the lowest term $\mathcal{R}_{0}$ in the semiclassical expansion of the density matrix for the Dirac hamiltonian (2.23) has been given in Eq. (2.53). It has been shown to contain separately the positive and negative energy contributions. The $\hbar$ order $\mathcal{R}_{1}$ and the $\hbar^{2}$ order $\mathcal{R}_{2}$ also have this property and can be written as

$$
\mathcal{R}_{1}=\mathcal{R}_{1}^{+}+\mathcal{R}_{1}^{-}, \quad \mathcal{R}_{2}=\mathcal{R}_{2}^{+}+\mathcal{R}_{2}^{-}
$$

We have found that the positive energy part of $\mathcal{R}_{1}$ is:

$$
\mathcal{R}_{1}^{+}=\mathcal{R}_{1, I}+\mathcal{R}_{1, \beta}+\mathcal{R}_{1, \alpha}+\mathcal{R}_{1, \gamma}
$$

with

$$
\begin{aligned}
& \mathcal{R}_{1, I}=\frac{1}{4 \varepsilon^{3}}(\varepsilon \delta+\Theta) \gamma^{5}(\alpha \cdot(\boldsymbol{p} \wedge \nabla V)), \\
& \mathcal{R}_{1, \beta}=\frac{1}{4 \varepsilon^{3}}(\varepsilon \delta+\Theta) \gamma^{5}\left(\boldsymbol{\gamma} \cdot\left(\boldsymbol{p} \wedge \nabla m^{*}\right)\right), \\
& \mathcal{R}_{1, \alpha}=0 \\
& \mathcal{R}_{1, \gamma}=-\frac{i}{4 \varepsilon^{3}}\left[m^{*}(\varepsilon \delta+\Theta)(\boldsymbol{\gamma} \cdot \nabla V)+\varepsilon^{2} \delta\left(\boldsymbol{\gamma} \cdot \nabla m^{*}\right)\right]
\end{aligned}
$$


In the above equations we have introduced the Dirac $\gamma^{5}$ and $\gamma$ matrices [Bj64]. The argument of the $\Theta$ function and its derivatives is $\lambda^{+}-\varepsilon-V$. From Eqs. (A.3)-(A.6), we see that $\mathcal{R}_{1}^{+}$has zero trace (when it is also taken over the spin degrees of freedom).

Using the same notation, the second-order term is:

$$
\mathcal{R}_{2}^{+}=\mathcal{R}_{2, I}+\mathcal{R}_{2, \beta}+\mathcal{R}_{2, \alpha}+\mathcal{R}_{2, \gamma}
$$

with

$$
\begin{aligned}
\mathcal{R}_{2, I}=\frac{1}{48 \varepsilon^{5}}[ & \left(\varepsilon^{2} \delta^{\prime \prime}+3 \varepsilon \delta^{\prime}+3 \delta\right)\left[\varepsilon^{2}(\nabla V)^{2}-(\boldsymbol{p} \cdot \nabla V)^{2}\right. \\
& \left.+m^{* 2}\left(\nabla m^{*}\right)^{2}+m^{*}(\boldsymbol{p} \cdot \nabla)^{2} m^{*}+\left(\boldsymbol{p} \cdot \nabla m^{*}\right)^{2}\right] \\
& +\left(\varepsilon^{3} \delta^{\prime \prime}+3 \varepsilon^{2} \delta^{\prime}+6 \varepsilon \delta+6 \Theta\right)\left[(\boldsymbol{p} \cdot \nabla)^{2} V+2 m^{*}\left(\nabla V \cdot \nabla m^{*}\right)\right] \\
& -3 \varepsilon^{2}\left(\varepsilon^{2} \delta^{\prime}+2 \varepsilon \delta+2 \Theta\right) \Delta V \\
& \left.-3 \varepsilon^{2} m^{*}\left(\varepsilon \delta^{\prime}+\delta\right) \Delta m^{*}\right] I \\
\mathcal{R}_{2, \beta}=\frac{1}{48 \varepsilon^{7}} & {\left[m^{*} \varepsilon^{2}\left(\varepsilon^{3} \delta^{\prime \prime}-3 \varepsilon \delta-3 \Theta\right)(\nabla V)^{2}\right.} \\
& -m^{*}\left(\varepsilon^{3} \delta^{\prime \prime}+6 \varepsilon^{2} \delta^{\prime}+15 \varepsilon \delta+15 \Theta\right)\left[(\boldsymbol{p} \cdot \nabla V)^{2}\right. \\
& \left.-m^{* 2}\left(\nabla m^{*}\right)^{2}-m^{*}(\boldsymbol{p} \cdot \nabla)^{2} m^{*}-\left(\boldsymbol{p} \cdot \nabla m^{*}\right)^{2}\right] \\
& -3 \varepsilon^{2}\left(\varepsilon^{2} \delta^{\prime}+3 \varepsilon \delta+3 \Theta\right)\left[2 m^{*}\left(\nabla m^{*}\right)^{2}+(\boldsymbol{p} \cdot \nabla)^{2} m^{*}\right] \\
& +m^{*} \varepsilon^{2}\left(\varepsilon^{2} \delta^{\prime \prime}+3 \varepsilon \delta^{\prime}+3 \delta\right)\left[(\boldsymbol{p} \cdot \nabla)^{2} V+2 m^{*}\left(\nabla V \cdot \nabla m^{*}\right)\right] \\
& -3 \varepsilon^{4}\left(\varepsilon \delta^{\prime}+\delta\right)\left[2\left(\nabla V \cdot \nabla m^{*}\right)+m^{*} \Delta V\right] \\
& \left.-3 \varepsilon^{2}\left(m^{* 2} \varepsilon^{2} \delta^{\prime}-3 p^{2} \varepsilon \delta-3 p^{2} \Theta\right) \Delta m^{*}\right] \beta \\
& \\
& \\
& \\
&
\end{aligned}
$$




$$
\begin{aligned}
& \mathcal{R}_{2, \alpha}=\frac{1}{48 \varepsilon^{7}[}[ \varepsilon^{2}\left(\varepsilon^{3} \delta^{\prime \prime}-3 \varepsilon \delta-3 \Theta\right)(\nabla V)^{2} \\
&-\left(\varepsilon^{3} \delta^{\prime \prime}+6 \varepsilon^{2} \delta^{\prime}+15 \varepsilon \delta+15 \Theta\right)\left[(\boldsymbol{p} \cdot \nabla V)^{2}\right. \\
&\left.-m^{* 2}\left(\nabla m^{*}\right)^{2}-m^{*}(\boldsymbol{p} \cdot \nabla)^{2} m^{*}-\left(\boldsymbol{p} \cdot \nabla m^{*}\right)^{2}\right] \\
&+\varepsilon^{2}\left(\varepsilon^{2} \delta^{\prime \prime}+3 \varepsilon \delta^{\prime}+3 \delta\right)\left[(\boldsymbol{p} \cdot \nabla)^{2} V+2 m^{*}\left(\nabla V \cdot \nabla m^{*}\right)\right] \\
&-3 \varepsilon^{4}\left(\varepsilon \delta^{\prime}+\delta\right) \Delta V \\
&\left.-3 m^{*} \varepsilon^{2}\left(\varepsilon^{2} \delta^{\prime}+3 \varepsilon \delta+3 \Theta\right) \Delta m^{*}\right](\boldsymbol{\alpha} \cdot \boldsymbol{p}) \\
&+\frac{1}{8 \varepsilon^{5}} {\left[\left(\varepsilon^{2} \delta^{\prime}+3 \varepsilon \delta+3 \Theta\right)[(\boldsymbol{p} \cdot \nabla V)(\boldsymbol{\alpha} \cdot \nabla V)\right.} \\
&\left.-\left(\boldsymbol{p} \cdot \nabla m^{*}\right)\left(\boldsymbol{\alpha} \cdot \nabla m^{*}\right)\right], \\
& \mathcal{R}_{2, \gamma}=\frac{i}{8 \varepsilon^{5}}\left(\varepsilon^{2} \delta^{\prime}+3 \varepsilon \delta+3 \Theta\right)\left(\boldsymbol{p} \cdot\left(\nabla V \wedge \nabla m^{*}\right)\right) \gamma^{5} \beta .
\end{aligned}
$$

The contributions of negative energy states are easily obtained from the positive terms making the following substitution:

$$
\begin{aligned}
& \mathcal{R}_{1}^{-}(\varepsilon)=\mathcal{R}_{1}^{+}\left(\varepsilon \longrightarrow-\varepsilon, \lambda^{+} \longrightarrow \lambda^{-}\right), \\
& \mathcal{R}_{2}^{-}(\varepsilon)=\mathcal{R}_{2}^{+}\left(\varepsilon \longrightarrow-\varepsilon, \lambda^{+} \longrightarrow \lambda^{-}\right) .
\end{aligned}
$$




\section{Appendix B}

AS DISCUSSED in Section 2.3, the idempotency property (2.54) of the density matrix $\hat{\mathcal{R}}$ results in some relations which the different orders of its semiclassical expansion have to satisfy, Eq. (2.55). We here only show how to prove that the well-known $\hbar^{2}$-order WK expansion of the density matrix $\hat{\rho}$ for a non-relativistic hamiltonian

$$
\hat{H}=\frac{\hat{p}^{2}}{2 m}+V(\boldsymbol{r})
$$

fulfils

$$
\left(\hat{\rho}^{2}\right)_{\mathrm{w}}=(\hat{\rho})_{\mathrm{w}} \equiv f
$$

up to order $\hbar^{2}$. This is an essential ingredient also for the relativistic generalization.

For the hamiltonian (B.1), we have [Je78]:

$$
H_{\mathrm{w}}=\frac{p^{2}}{2 m}+V
$$

and

$$
f=f_{0}+\hbar^{2} f_{2}
$$

with

$$
f_{0}=\Theta\left(\lambda-H_{\mathrm{w}}\right)
$$




$$
\begin{aligned}
f_{2}= & -\frac{1}{8 m} \Delta V \delta^{\prime}\left(\lambda-H_{\mathrm{w}}\right) \\
& +\frac{1}{24 m}\left[(\nabla V)^{2}+\frac{1}{m}(\boldsymbol{p} \cdot \nabla)^{2} V\right] \delta^{\prime \prime}\left(\lambda-H_{\mathrm{w}}\right) .
\end{aligned}
$$

At order $\hbar^{0}$ it is evident that $f_{0}^{2}=f_{0}$. At second order, using Eq. (2.7):

$$
\begin{aligned}
\left(\hat{\rho}^{2}\right)_{\mathrm{w}}= & \left(f_{0}+\hbar^{2} f_{2}\right)\left(1+\frac{i \hbar}{2} \overleftrightarrow{\Lambda}-\frac{\hbar^{2}}{8} \overleftrightarrow{\Lambda}^{2}\right)\left(f_{0}+\hbar^{2} f_{2}\right) \\
= & f_{0}^{2}+\hbar^{2}\left(2 f_{0} f_{2}-\frac{1}{8} f_{0} \overleftrightarrow{\Lambda}^{2} f_{0}\right) \\
= & \Theta-\frac{\hbar^{2}}{4 m}\left[\Delta V\left(\Theta \delta^{\prime}+\delta^{2}\right)\right. \\
& \left.\quad-\left((\nabla V)^{2}+\frac{1}{m}(\boldsymbol{p} \cdot \nabla)^{2} V\right)\left(\frac{1}{3} \Theta \delta^{\prime \prime}+\delta \delta^{\prime}\right)\right] .
\end{aligned}
$$

To compare Eq. (B.7) with (B.6), it is necessary to interpret the products of the step function and its derivatives which appear in (B.7). For that purpose, let us recall two properties of distributions [Go67]:

$$
\begin{aligned}
\left\langle T^{(n)}, \varphi\right\rangle & =(-1)^{n}\left\langle T, \varphi^{(n)}\right\rangle, \\
\langle\phi T, \varphi\rangle & =\langle T, \phi \varphi\rangle,
\end{aligned}
$$

where $T$ is a distribution (strictly speaking, a continuous linear functional on the space $\mathcal{D}$ of the base functions [Go67]), $T^{(\mathfrak{n})}$ is its $n$ th-derivative, $\varphi \in \mathcal{D}$, and $\phi$ is a function of the class $C_{\infty} .\langle T, \varphi\rangle$ denotes the scalar which results from the action of $T$ on $\varphi$. For example, $\left\langle\delta^{(n)}, \varphi\right\rangle=(-1)^{n} \varphi^{(n)}(0)$. From (B.8) it is easy to show that

$$
\begin{aligned}
\langle\Theta \delta, \varphi\rangle & =-\langle\Theta \delta, \varphi\rangle+\langle\delta, \varphi\rangle \\
\left\langle\Theta \delta^{\prime}, \varphi\right\rangle & =-\left\langle\Theta \delta^{\prime}, \varphi\right\rangle-2\left\langle\delta^{2}, \varphi\right\rangle+\left\langle\delta^{\prime}, \varphi\right\rangle, \\
\left\langle\Theta \delta^{\prime \prime}, \varphi\right\rangle & =-\left\langle\Theta \delta^{\prime \prime}, \varphi\right\rangle-6\left\langle\delta \delta^{\prime}, \varphi\right\rangle+\left\langle\delta^{\prime \prime}, \varphi\right\rangle .
\end{aligned}
$$


Then, we formally have:

$$
\begin{aligned}
\Theta \delta & =\frac{1}{2} \delta, \\
\Theta \delta^{\prime}+\delta^{2} & =\frac{1}{2} \delta^{\prime}, \\
\frac{1}{3} \Theta \delta^{\prime \prime}+\delta \delta^{\prime} & =\frac{1}{6} \delta^{\prime \prime} .
\end{aligned}
$$

Inserting (B.10) into (B.7) we prove (B.2) to order $\hbar^{2}$ (we mention that a more indirect proof has been given in [Ha84], p. 191). The verification that the relativistic density matrix given in Appendix A fulfils Eq. (2.55) follows along the same lines, and only the relations (B.10) are required. 


\section{Appendix C}

IN SECTION 2.4 the integration over the momentum $p$, needed in order to get the WK expansion of $\rho, \rho_{\mathbf{s}}, \tau$ and $e$, could be performed algebraically in closed form because we introduced the assumption that the corresponding integrands depend on $p$ only through $p$, i.e., that they are invariant under rotation in momentum space. In particular, denoting the angular average of a quantity $f(p)$ by

$$
\langle f\rangle(p)=\frac{1}{4 \pi} \int d \Omega f(\boldsymbol{p}),
$$

we made use of the following equalities:

$$
\begin{aligned}
\left\langle(\boldsymbol{p} \cdot \nabla V)^{2}\right\rangle & =\frac{1}{3} p^{2}(\nabla V)^{2} \\
\left\langle\left(\boldsymbol{p} \cdot \nabla m^{*}\right)^{2}\right\rangle & =\frac{1}{3} p^{2}\left(\nabla m^{*}\right)^{2} \\
\left\langle(\boldsymbol{p} \cdot \nabla)^{2} V\right\rangle & =\frac{1}{3} p^{2} \Delta V \\
\left\langle(\boldsymbol{p} \cdot \nabla)^{2} m^{*}\right\rangle & =\frac{1}{3} p^{2} \Delta m^{*}
\end{aligned}
$$

and

$$
\langle\boldsymbol{p} \cdot \nabla V\rangle=\left\langle\boldsymbol{p} \cdot \nabla m^{*}\right\rangle=0
$$


To obtain the WK densities, after angular averaging, one has to calculate integrals of the type

$$
\begin{aligned}
F(n ; \mathrm{a}, \mathrm{b}) & =\int d p \frac{p^{\mathrm{a}}}{\left(p^{2}+m^{* 2}\right)^{\mathrm{b} / 2}} \frac{d^{\mathrm{n}} \Theta}{d \lambda^{n}} \\
& =\frac{d^{n}}{d \lambda^{n}} \int_{0}^{k_{\mathrm{F}}} d p \frac{p^{\mathrm{a}}}{\left(p^{2}+m^{* 2}\right)^{\mathrm{b} / 2}}
\end{aligned}
$$

where $\Theta=\Theta(\lambda-V-\varepsilon)$ and $k_{F}=\left((\lambda-V)^{2}-m^{* 2}\right)^{1 / 2}$ depend on $\lambda$. For the cases $n=1,2,3$ (and a, b integer) needed in the $\hbar^{2}$-order expansion, compact expressions can be given:

$$
\begin{aligned}
F(1 ; \mathrm{a}, \mathrm{b})= & \frac{k_{\mathrm{F}}^{\mathrm{a}-1}}{\varepsilon_{\mathrm{F}}^{\mathrm{b}-1}} \\
F(2 ; \mathrm{a}, \mathrm{b})= & \frac{k_{\mathrm{F}}^{\mathrm{a}-3}}{\varepsilon_{\mathrm{F}}^{\mathrm{b}}}\left[(\mathrm{a}-1) \varepsilon_{\mathrm{F}}^{2}-(\mathrm{b}-1) k_{\mathrm{F}}^{2}\right] \\
F(3 ; \mathrm{a}, \mathrm{b})= & \frac{k_{\mathrm{F}}^{\mathrm{a}-5}}{\varepsilon_{\mathrm{F}}^{\mathrm{b}+1}}\left[\left(\mathrm{a}^{2}-4 \mathrm{a}+3\right) \varepsilon_{\mathrm{F}}^{4}+(3 \mathrm{a}-2 \mathrm{ab}+2 \mathrm{~b}-3) \varepsilon_{\mathrm{F}}^{2} k_{\mathrm{F}}^{2}\right. \\
& \left.\quad+\left(\mathrm{b}^{2}-\mathrm{b}\right) k_{\mathrm{F}}^{4}\right] .
\end{aligned}
$$

For $n=0$, one can make use of the relations

$$
F(0 ; \mathrm{a}, \mathrm{b})=F(0 ; \mathrm{a}-2, \mathrm{~b}-2)-m^{* 2} F(0 ; \mathrm{a}-2, \mathrm{~b})
$$

and if $b \neq 2$

$$
F(0 ; \mathrm{a}, \mathrm{b})=\frac{1}{2-b} k_{\mathrm{F}}^{\mathrm{a}-1} \varepsilon_{\mathrm{F}}^{2-\mathrm{b}}+\frac{\mathrm{a}-1}{\mathrm{~b}-2} F(0 ; \mathrm{a}-2, \mathrm{~b}-2),
$$

to reduce the integrals to one of the elementary cases

$$
\begin{aligned}
& F(0 ; 0,1)=\ln \frac{k_{\mathrm{F}}+\varepsilon_{\mathrm{F}}}{m^{*}} \\
& F(0 ; 0,2)=\frac{1}{m^{*}} \arctan \frac{k_{\mathrm{F}}}{m^{*}} .
\end{aligned}
$$




\section{Bibliography}

[Ab65] M. Abramowitz and I.A. Stegun, Handbook of Mathematical Functions (Dover, New York, 1965).

[An83] M.R. Anastasio, L.S. Celenza, W.S. Pong and C.M. Shakin, Phys. Rep. 100 (1983) 327.

[Ar87] R. Arvieu, M. Durand, P. Schuck and E. Suraud (eds.), International Workshop on Semiclassical and Phase Space Approaches to the Dynamics of the Nucleus, J. de Phys. Colloque C2 (1987).

[Ba71] R. Balian and C. Bloch, Ann. Phys. 63 (1971) 592.

[Ba81a] M. Barranco and J.R. Buchler, Phys. Rev. C24 (1981) 1191.

[Ba81b] M. Barranco and J. Treiner, Nucl. Phys. A351 (1981) 269.

[Ba82] J. Bartel, P. Quentin, M. Brack, C. Guet and H.-B. Håkansson, Nucl. Phys. A386 (1982) 79.

[Ba84] N.L. Balazs and B.K. Jennings, Phys. Rep. 104 (1984) 347.

[Ba85] J. Bartel, M. Brack and M. Durand, Nucl. Phys. A445 (1985) 263.

[Ba91] M. Barranco, R.J. Lombard, S. Marcos and S.A. Moszkowski, Phys. Rev. C44 (1991) 178.

[Be53] H.A. Bethe, Sci. Am. 189(3) (1953) 58.

[Be56] R.A. Berg and L. Wilets, Phys. Rev. 101 (1956) 201. 
[Be75] M. Beiner, H. Flocard, Nguyen Van Giai and P. Quentin, Nucl. Phys. A238 (1975) 29.

[Be88] G.F. Bertsch and S. Das Gupta, Phys. Rep. 160 (1988) 190.

[Bh71] R.K. Bhaduri and C.K. Ross, Phys. Rev. Lett. 27 (1971) 606.

[Bj64] J.D. Bjorken and S.D. Drell, Relativistic Quantum Mechanics (McGraw-Hill, New York, 1964).

[Bl80] J.P. Blaizot, Phys. Rep. 64 (1980) 171.

[B187] P.G. Blunden and M.J. Iqbal, Phys. Lett. B196 (1987) 295.

[Bo69] A. Bohr and B.R. Mottelson, Nuclear Structure, Vol. 1 (Benjamin, New York, 1969).

[Bo76a] O. Bohigas, X. Campi, H. Krivine and J. Treiner, Phys. Lett. B64 (1976) 381.

[Bo76b] P. Bonche, S. Koonin and J.W. Negele, Phys. Rev. C13 (1976) 1226.

[Bo77a] J. Boguta and J. Rafelski, Phys. Lett. B71 (1977) 22.

[Bo77b] J. Boguta and A.R. Bodmer, Nucl. Phys. A292 (1977) 413.

[Bo82] J. Boguta, Phys. Lett. B109 (1982) 251.

[Bo83a] J. Boguta and H. Stöcker, Phys. Lett. B120 (1983) 289.

[Bo83b] J. Boguta and S.A. Moszkowski, Nucl. Phys. A403 (1983) 445.

[Bo84a] A. Bouyssy, S. Marcos and J.-F. Mathiot, Nucl. Phys. A415 (1984) 497.

[Bo84b] A. Bouyssy, S. Marcos and Pham Van Thieu, Nucl. Phys. A422 (1984) 541.

[Bo87] A. Bouyssy, J.-F. Mathiot, Nguyen Van Giai and S. Marcos, Phys. Rev. C36 (1987) 380 .

[Bo89] A.R. Bodmer and C.E. Price, Nucl. Phys. A505 (1989) 123.

[Bo91] H.F. Boersma, R. Malfliet and O. Scholten, Phys. Lett. B269 (1991) 1.

[Br68a] K.A. Brueckner, J.R. Buchler, S. Jorna and R.J. Lombard, Phys. Rev. 171 (1968) 1188.

[Br68b] K.A. Brueckner, J.R. Buchler and M.M. Kelly, Phys. Rev. 173 (1968) 944.

[Br72] M. Brack, Jens Damgaard, A.S. Jensen, H.C. Pauli, V.M. Strutinsky and C.Y. Wong, Rev. Mod. Phys. 44 (1972) 320. 
[Br73] M. Brack and H.C. Pauli, Nucl. Phys. A207 (1973) 401.

[Br76] M. Brack, B.K. Jennings and Y.H. Chu, Phys. Lett. B65 (1976) 1.

[Br81] M. Brack and P. Quentin, Nucl. Phys. A361 (1981) 35.

[Br84] R. Brockmann and R. Machleidt, Phys. Lett. B149 (1984) 283.

[Br85] M. Brack, C. Guet and H.-B. Håkansson, Phys. Rep. 123 (1985) 275.

[Br90] R. Brockmann and R. Machleidt, Phys. Rev. C42 (1990) 1965.

[Br92] R. Brockmann and H. Toki, Phys. Rev. Lett. 68 (1992) 3408.

[Bu84] M. Buenerd, A. Lounis, J. Chauvin, D. Lebrun, P. Martin, G. Duhamel, J.C. Gondrand and P. de Saintignon, Nucl. Phys. A424 (1984) 313.

[Ce86] L. S. Celenza and C. M. Shakin, Relativistic Nuclear Physics: Theories of Structure and Scattering (World Scientific, Singapore, 1986).

[Ce90a] M. Centelles, M. Pi, X. Viñas, F. Garcias and M. Barranco, Nucl. Phys. A510 (1990) 397.

[Ce90b] M. Centelles, X. Viñas, M. Barranco and P. Schuck, Nucl. Phys. A519 (1990) $73 c$.

[Ce91] M. Centelles, X. Viñas, M. Barranco, N. Ohtsuka, Amand Faessler, Dao Tien Khoa and H. Müther, J. Phys. G17 (1991) L193.

[Ce92a] M. Centelles, X. Viñas, M. Barranco, S. Marcos and R.J. Lombard, Nucl. Phys. A537 (1992) 486.

[Ce92b] M. Centelles, X. Viñas, M. Barranco, N. Ohtsuka, Amand Faessler, Dao Tien Khoa and H. Müther, Barcelona University preprint (December 1991).

[Ce92c] M. Centelles, X. Viñas, M. Barranco and P. Schuck, Barcelona University preprint (February 1992).

[Ch74] S.A. Chin and J.D. Walecka, Phys. Lett. B52 (1974) 24.

[Ch77] S.A. Chin Ann. Phys. 108 (1977) 301.

[Cl83] B.C. Clark, S. Hama, R.L. Mercer, L. Ray and B.D. Serot, Phys. Rev. Lett. 50 (1983) 1644.

[Co74] S. Cohen, F. Plasil and W.J. Swiatecki, Ann. Phys. 82 (1974) 557. 
[Cu85] R.Y. Cusson, P.-G. Reinhard, J.J. Molitoris, H. Stöcker, M.R. Strayer and W. Greiner, Phys. Rev. Lett. 55 (1985) 2786.

[Da80] K.T.R. Davies, H. Flocard, S. Krieger and M.S. Weiss, Nucl. Phys. A342 (1980) 111.

[Da81] K.T.R. Davies and-S.E. Koonin, Phys. Rev. C23 (1981) 2042.

[Da85a] D. Dalili, J. Németh and C. Ngô, Z. Phys. A321 (1985) 335.

[Da85b] D. Dalili, Ph.D. Thesis, Université Paris-Sud, Orsay, 1985.

[Di89] J. Diaz, J.L. Ferrero, J.A. Ruiz, B. Bilwes and R. Bilwes, Nucl. Phys. A494 (1989) 311.

[Do79] A.H. MacDonald and S.H. Vosko, J. Phys. C12 (1979) 2977.

[Dr85] R.M. Dreizler and J. da Providencia (eds.), Density Functional Methods in Physics, Vol. 123 NATO ASI Series B (Plenum, New York, 1985).

[Dr90] R.M. Dreizler and E.K.U. Gross, Density Functional Theory (Springer-Verlag, Berlin, 1990).

[Du56] H.P. Duerr, Phys. Rev. 103 (1956) 469.

[Ei91a] D. Von-Eiff and M.K. Weigel, Z. Phys. A339 (1991) 63.

[Ei91b] D. Von-Eiff, S. Haddad and M.K. Weigel, Lawrence Berkeley Laboratory preprint LBL-31614 (December 1991), submitted to Phys. Rev. C.

[E190] H. Elsenhans, H. Müther and R. Machleidt, Nucl. Phys. A515 (1990) 715.

[En87] E. Engel and R.M. Dreizler, Phys. Rev. A35 (1987) 3607.

[En89] E. Engel, H. Müller and R.M. Dreizler, Phys. Rev. A39 (1989) 4873.

[Fe91] H. Feldmeier and J. Lindner, Z. Phys. A341 (1991) 83.

[Fl75] H. Flocard, Ph.D. Thesis, Université Paris-Sud, Orsay, 1975.

[Fr75] J.L. Friar and J.W. Negele, Adv. Nucl. Phys. 8 (1975) 219.

[Ga89a] F. Garcias, M. Barranco, J. Németh, C. Ngô and X. Viñas, Nucl. Phys. A495 (1989) $169 \mathrm{c}$.

[Ga89b] F. Garcias, Ph.D. Thesis, Universitat de ses Illes Balears, 1989.

[Ga90a] Y.K. Gambhir, P. Ring and A. Thimet, Ann. Phys. 198 (1990) 132. 
[Ga90b] F. Garcias, M. Barranco, Amand Faessler and N. Ohtsuka, Z. Phys. A336 (1990) 31.

[Ga91] J. Gallego and S. Das Gupta, Phys. Lett. B270 (1991) 6.

[Gl83] N.K. Glendenning, B. Banerjee and M. Gyulassy, Ann. Phys. 149 (1983) 1.

[G187] N.K. Glendenning, Phys. Lett. B185 (1987) 275.

[Gl89] N.K. Glendenning, Nucl. Phys. A493 (1989) 521.

[G190] P. Gleissl, M. Brack, J. Meyer and P. Quentin, Ann. Phys. 197 (1990) 205.

[Go67] R. Gouyon, Intégration et Distributions (Vuibert, Paris, 1967), p. $71 \mathrm{ff}$.

[Go75] D. Gogny, Proc. Int. Conf. on Nuclear Self-consistent Fields, Trieste, 1975, eds. G. Ripka and M. Porneuf (North Holland, Amsterdam, 1975).

[Gr79] B. Grammaticos and A. Voros, Ann. Phys. 123 (1979) 359.

[Gr80] B. Grammaticos and A. Voros, Ann. Phys. 129 (1980) 153.

[Gr85] W. Greiner, B. Müller and J. Rafelski, Quantum Electrodynamics of Strong Fields (Springer-Verlag, Berlin, 1985).

[Gr87] C. Grégoire, B. Rémaud, F. Sébille, L. Vinet and Y. Raffray, Nucl. Phys. A465 (1987) 317.

[Gu92] A. Guirao, X. Viñas and J. Díaz, Nucl. Phys. A (1992), in print.

[Ha84] R.W. Hasse, R. Arvieu and P. Schuck (eds.), Workshop on Semiclassical Methods in Nuclear Physics, J. de Phys. Colloque C6 (1984).

[Ha87] B. ter Haar and R. Malflet, Phys. Rep. 149 (1987) 207.

[He84] A.C. Hearn, DU User's Manual, Version 3.1, Rand Publication CP78 (revised 4/84).

[Hi91] D. Hirata, H. Toki, T. Watabe, I. Tanihata and B.V. Carlson, Phys. Rev. C44 (1991) 1467.

[Ho64] P. Hohenberg and W. Kohn, Phys. Rev. 136 (1964) B864.

[Ho73] C.H. Hodges, Can. J. Phys. 51 (1973) 1428.

[Ho81a] C.J. Horowitz and J.D. Walecka, Nucl. Phys. A364 (1981) 429.

[Ho81b] C.J. Horowitz and B.D. Serot, Nucl. Phys. A368 (1981) 503.

[Ho84] C.J. Horowitz and B.D. Serot, Phys. Lett. B140 (1984) 181. 
[Ho87] C.J. Horowitz and B.D. Serot, Nucl. Phys. A464 (1987) 613.

[Ho88a] U. Hofmann and P. Ring, Phys. Lett. B214 (1988) 307.

[Ho88b] J.Y. Hostachy, M. Buenerd, J. Chauvin, D. Lebrun, Ph. Martin, J.C. Lugol, L. Papineau, P. Roussel, N. Alamanos, J. Arvieux and C. Cerruti, Nucl. Phys. A490 (1988) 441.

[Ho89] D. Hofer and W. Stocker, Nucl. Phys. A492 (1989) 637.

[Hu90] M. L'Huillier, Nguyen Van Giai and N. Auerbach, Nucl. Phys. A519 (1990) 83c.

[Je73] B.K. Jennings, Nucl. Phys. A207 (1973) 538.

[Je75a] B.K. Jennings and R.K. Bhaduri, Nucl. Phys. A237 (1975) 149.

[Je75b] B.K. Jennings, R.K. Bhaduri and M. Brack, Nucl. Phys. A253 (1975) 29.

[Je78] B.K. Jennings, Phys. Lett. B74 (1978) 13.

[Jo55] M.H. Johnson and E. Teller, Phys. Rev. 98 (1955) 783.

[Jo87] C.H. Johnson, D.J. Horen and C. Mahaux, Phys. Rev. C36 (1987) 2252.

[Jo89] R.O. Jones and O. Gunnarsson, Rev. Mod. Phys. 61 (1989) 689.

[Kh81] S.B. Khadkikar, L. Rikus, Amand Faessler and R. Sartor, Nucl. Phys. A369 (1981) 495.

[Ki33] J.G. Kirkwood, Phys. Rev. 44 (1933) 31.

[Ki67] D.A. Kirzhnits, Field Theoretical Methods in Many-Body Systems (Pergamon, Oxford, 1967).

[Ko91] W. Koepf, M.M. Sharma and P. Ring, Nucl. Phys. A533 (1991) 95.

[Kr90] H. Krivine, M. Casas and J. Martorell, Ann. Phys. 200 (1990) 304.

[Ku91a] H. Kucharek and P. Ring, Z. Phys. A339 (1991) 23.

[Ku91b] V.I. Kukulin, G. Loyola and M. Moshinsky, Phys. Lett. A158 (1991) 19.

[Le84] S. Levit, Phys. Lett. B139 (1984) 147.

[Le86] S.-J. Lee, J. Fink, A.B. Balantekin, M.R. Strayer, A.S. Umar, P.-G. Reinhard, J.A. Maruhn and W. Greiner, Phys. Rev. Lett. 57 (1986) 2916;

Phys. Rev. Lett. 59 (1987) 1171.

[Li89] E. Lipparini and S. Stringari, Phys. Rep. 175 (1989) 103. 
[Lo73] R.J. Lombard, Ann. Phys. 77 (1973) 380.

[Lo88] M. Lopez-Quelle, S. Marcos, R. Niembro, A. Bouyssy and Nguyen Van Giai, Nucl. Phys. A483 (1988) 479.

[Lo91] R.J. Lombard, D. Mas and S.A. Moszkowski, J. Phys. G17 (1991) 455.

[Lu83] S. Lundqvist and N.H. March (eds.), Theory of the Inhomogeneous Electron Gas (Plenum, New York, 1983).

[Ma78] M.H. Macfarlane and S.C. Pieper, Argonne National Laboratory report ANL76-11 (1978).

[Ma82] S. Marcos, M. Barranco and J.R. Buchler, Nucl. Phys. A381 (1982) 507.

[Ma85] R. Machleidt and R. Brockmann, Phys. Lett. B160 (1985) 207.

[Ma86] P. Malzacher and R.M. Dreizler, Z. Phys. D2 (1986) 37.

[Ma89] R. Machleidt, Adv. Nucl. Phys. 19 (1989) 189.

[Ma91] S. Marcos, M. López-Quelle and Nguyen Van Giai, Phys. Lett. B257 (1991) 5 .

[Me65] N.D. Mermin, Phys. Rev. 137 (1965) A1441.

[Mø88] P. Möller, W.D. Myers, W.J. Swiatecki and J. Treiner, At. Data Nucl. Data Tables 39 (1988) 225.

[Mü81] K.-H. Müller, Nucl. Phys. A372 (1981) 459.

[Mu81] D.R. Murphy, Phys. Rev. A24 (1981) 1682.

[Mü87] H. Müther, M. Prakash and T.L. Ainsworth, Phys. Lett. B199 (1987) 469.

[Mü88] H. Müther, R. Machleidt and R. Brockmann, Phys. Lett. B202 (1988) 483.

[Mü89] H. Müller, E. Engel and R.M. Dreizler, Phys. Rev. A40 (1989) 5542.

[Mü90] H. Müther, R. Machleidt and R. Brockmann, Phys. Rev. C42 (1990) 1981.

[Mü92] H. Müller and R.M. Dreizler, Z. Phys. A341 (1992) 417.

[My69] W.D. Myers and W.J. Swiatecki, Ann. Phys. 55 (1969) 395.

[My74] W.D. Myers and W.J. Swiatecki, Ann. Phys. 84 (1974) 186.

[My77] W.D. Myers, Droplet Model of Atomic Nuclei (IFI/Plenum, New York, 1977).

[My91] W.D. Myers and W.J. Swiatecki, Ann. Phys. 211 (1991) 292. 
[Ne70] J.W. Negele, Phys. Rev. C1 (1970) 1260.

[Ne82] J.W. Negele, Rev. Mod. Phys. 54 (1982) 913.

[Ne83] J.A. McNeil, J.R. Shepard and S.J. Wallace, Phys. Rev. Lett. 50 (1983) 1439.

[Ng75] C. Ngô, B. Tamain, M. Beiner, R.J. Lombard, D. Mas and H.H. Deubler, Nucl. Phys. A252 (1975) 237.

[Oh87] N. Ohtsuka, R. Linden, Amand Faessler and F.B. Malik, Nucl. Phys. A465 (1987) 550.

[Oh88] N. Ohtsuka, M. Shabshiry, R. Linden, H. Müther and Amand Faessler, Nucl. Phys. A490 (1988) 715.

[Pa87] W. Pannert, P. Ring and J. Boguta, Phys. Rev. Lett. 59 (1987) 2420.

[Pe91] I. Zh. Petkov and M.V. Stoitsov, Nuclear Density Functional Theory (Clarendon Press, Oxford, 1991).

[Po91] W.F. Pohlner and R.M. Dreizler, Phys. Rev. A44 (1991) 7165.

[Pr81] M. Prakash, S. Shlomo and V.M. Kolomietz, Nucl. Phys. A370 (1981) 30.

[Pr83] J.P. da Providência and G. Holzwarth, Nucl. Phys. A398 (1983) 59.

[Pr85] C.E. Price and G.E. Walker, Phys. Lett. B155 (1985) 17.

[Pr87] C.E. Price and G.E. Walker, Phys. Rev. C36 (1987) 354.

[Qu78] P. Quentin and H. Flocard, Ann. Rev. Nucl. Part. Sci. 28 (1978) 523.

[Ra73] A.K. Rajagopal and J. Callaway, Phys. Rev. B7 (1973) 1912.

[Ra82] M. Rayet, M. Arnould, F. Tondeur and G. Paulus, Astron. Astrophys. 116 (1982) 183.

[Ra83] M.V. Ramana and A.K. Rajagopal, Adv. Chem. Phys. 54 (1983) 231.

[Ra87] G. Rayna, DU: Software for Algebraic Computation (Springer, New York, 1987).

[Ra90] J. Ramschütz, F. Weber and M.K. Weigel, J. Phys. G16 (1990) 987.

[Re86] P.-G. Reinhard, M. Rufa, J. Maruhn, W. Greiner and J. Friedrich, Z. Phys. A323 (1986) 13.

[Re88] P.-G. Reinhard, Z. Phys. A329 (1988) 257.

[Re89] P.-G. Reinhard, Rep. Prog. Phys. 52 (1989) 439. 
[Ri80] P. Ring and P. Schuck, The Nuclear Many-Body Problem (Springer-Verlag, New York, 1980), ch. 13.

[Ri88] P. Ring, in Proc. Workshop on Nuclear Structure with Medium Energy Probes (Santa Fe, New Mexico, October 1988).

[Ro85] P. Roussel, N. Alamanos, F. Auger, J. Barrette, B. Berthier, B. Fernandez, ..-

L. Papineau, H. Doubre and W. Mittig, Phys. Rev. Lett. 54 (1985) 1779.

[Ro87] P. Roussel, J. Barrette, F. Auger, B. Berthier, B. Fernandez, J. Gastebois, A. Gillibert, L. Papineau, W. Mittig, D. Disdier, B. Lott, V. Rauch, F. Scheibling, C. Stephan and L. Tassan-Got, Phys. Lett. B185 (1987) 29.

[Sc89] P. Schuck, R.W. Hasse, J. Jaenicke, C. Grégoire, B. Rémaud, F. Sébille and E. Suraud, Prog. Part. Nucl. Phys. 22 (1989) 181.

[Sc91] K.W. Schmid, H. Müther and R. Machleidt, Nucl. Phys. A530 (1991) 14.

[Se78] F.E. Serr and J. D. Walecka, Phys. Lett. B79 (1978) 10.

[Se79] B.D. Serot and J.D. Walecka, Phys. Lett. B87 (1979) 172.

[Se86] B.D. Serot and J.D. Walecka, Adv. Nucl. Phys. 16 (1986) 1.

[Sh88] M.M. Sharma, W.T.A. Borghols, S. Brandenburg, S. Crona, J.H. Meier, M. Harakeh and A. van der Woude, Phys. Rev. C38 (1988) 2562.

[Sh91a] M.M. Sharma, S.A. Moszkowski and P. Ring, Phys. Rev. C44 (1991) 2493.

[Sh91b] M.M. Sharma and P. Ring, ISN Grenoble preprint 91.08, 1991.

[Si86] A.J. Sierk, Phys. Rev. C33 (1986) 2039.

[Sk56] T.H.R. Skyrme, Phil. Mag. 1 (1956) 1043.

[Sm71] G.B. Smith and L.J. Tassie, Ann. Phys. 65 (1971) 352.

[Sp72] D.W.L. Sprung, Adv. Nucl. Phys. 5 (1972) 225.

[Sp92] C. Speicher, R.M. Dreizler and E. Engel, Ann. Phys. 213 (1992) 312.

[St67] V.M. Strutinsky, Nucl. Phys. A95 (1967) 420.

[St68] V.M. Strutinsky, Nucl. Phys. A122 (1968) 1.

[St85] W. Stocker and M. Farine, Ann. Phys. 159 (1985) 255.

[St88] W. Stocker, J. Bartel, J.R. Nix and A.J. Sierk, Nucl. Phys. A489 (1988) 252.

[St91] W. Stocker and M.M. Sharma, Z. Phys. A339 (1991) 147. 
[Su87] E. Suraud, Nucl. Phys. A462 (1987) 109.

[Su88] E. Suraud, M. Barranco and J. Treiner, Nucl. Phys. A480 (1988) 29.

[Su89] E. Suraud, M. Pi and P. Schuck, Nucl. Phys. A492 (1989) 294.

[Ta92] K. Taruishi and P. Schuck, Z. Phys. A (1992), to be published.

[Te90] R. Tegen, Ann. Phys. 197 (1990) 439.

[Tr86] J. Treiner and H. Krivine, Ann. Phys. 170 (1986) 406.

[Uh36] G.E. Uhlenbeck and E. Beth, Physica 3 (1936) 729.

[Va32] M.S. Vallarta and N. Rosen, Phys. Rev. 41 (1932) 708.

[Va72] D. Vautherin and D.M. Brink, Phys. Rev. C5 (1972) 626.

[Wa74] J.D. Walecka, Ann. Phys. 83 (1974) 491.

[We89] F. Weber and M.K. Weigel, Nucl. Phys. A493 (1989) 549.

[We91] M.K. Weigel, S. Haddad and F. Weber, J. Phys. G17 (1991) 619.

[Wi32] E. Wigner, Phys. Rev. 40 (1932) 749.

[Zh91a] J.-K. Zhang and D.S. Onley, Nucl. Phys. A526 (1991) 245.

[Zh91b] Z.Y. Zhu, H.J. Mang and P. Ring, Phys. Lett. B254 (1991) 325.

[Zi90] J. Zimanyi and S.A. Moszkowski, Phys. Rev. C42 (1990) 1416. 
